\title{
EVALUATION OF THE ADOM CLOUD MODULE
}

\author{
by \\ Anna Glazer

\begin{abstract}
A thesis submitted to
the Faculty of Graduate Studies and Research

in partial fulfillment of the

requirements for the degree of

MASTER OF SCIENCE
\end{abstract}
Department of Atmospheric and Oceanic Sciences
McGill University
Montreal, Quebec

March, 1992

@ Anna Glazer, 1992 


\section{ABSTRACT}

The Acid Deposition and Oxidant Model (ADOM) is an Eulerian long-range transport and deposition model. One of the most highly parametrized and least well established parts of the model is the cloud module that describes cloud formation, pollutant scavenging, aqueous-phase chemistry and wet deposition. As a means of gaining insight into the cloud module, results from simulations with the module are compared with the results of simulations for equivalent conditions with a threedimensional dynamic cloud chemistry model.

Comparisons of results for a variety of initial conditions show that wetdeposition of sulphate, nitrate and ammonium ions tend to be underpredicted by the cloud module and that the $\mathrm{pH}$ of the rain is overpredicted. However, the differences are for the most part not large. Concentrations of hydrogen peroxide deposited at the surface are significantly smaller in the ADOM module than in the cloud chemistry model. The results of the cloud module do seem to be sensitive to the model cloud top height. 


\section{RESUMÉ}

L'ADOM, un modèle décrivant l'oxydation et la déposition des acides, est un modèle eulerien qui appartient à la classe des modèles de transport à longuedistance. La partie la plus parametrisée de ce modèle, donc soumise aux plus importantes incertitudes, c'est le module des nuages qui décrit la formation des nuages, le lessivage, les réactions chimiques dans la phase liquide et la déposition humide. Pour évaluer le module des nuages, les resultats obtenus sont comparés avec ceux d'un autre modele de chimie des nuages, plus élaboré et à trois dimensions, initialisé avec les données equivalentes.

Les comparaisons qui ont été faites pour une multitude de conditions initiales démontrent que la déposition humide des sulphates, nitrates et ammonium est sousestimée par le module alors que le $\mathrm{pH}$ de la pluie est surestimé. Cependant les differences ne sont pas très importantes. Par contre la quantité de peroxyde d'hydrogène déposée à la surface est beaucoup plus petite dans le module d'ADOM que dans le modèle à trois dimensions. En plus les resultats des simulations sont fortement influencés par la position du sommet des nuages. 


\section{ACKNOWLEDGEMENTS}

It is a great pleasure to express my deep appreciation to Prof. Henry Leighton, my thesis advisor, for his patient guidance, continuous interest and helpful encouragement throughout all this study.

I would also like to thank Prof. Peter Yau for his help in running the cloud dynamics model.

Finally, I wish to acknowledge the Atmospheric Environment Service for its financial support of this work in the form of a science subvention. 


\section{TABLE OF CONTENTS}

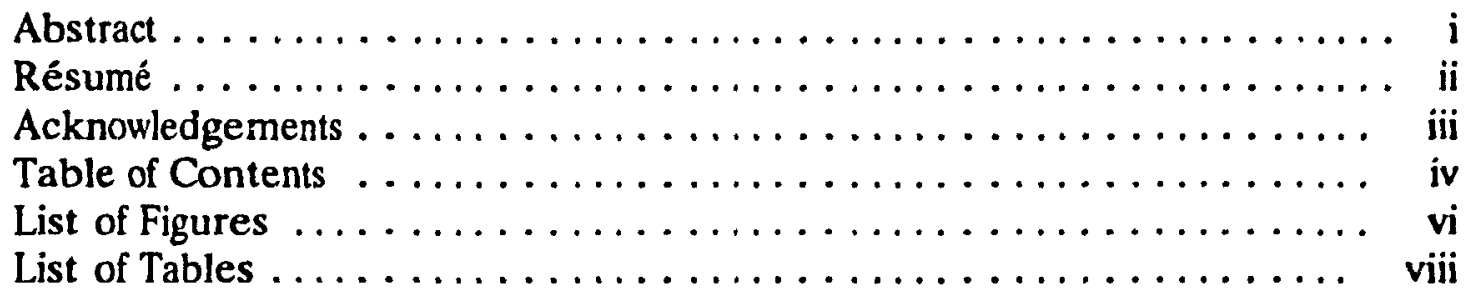

Chapter 1 INTRODUCTION $\ldots \ldots \ldots \ldots \ldots \ldots \ldots \ldots \ldots \ldots, 1$

1.1 Overview ........................... 1

$1.2 \quad$ Purpose of this study $\ldots \ldots \ldots \ldots \ldots \ldots \ldots \ldots \ldots$

Chapter 2 MODEL DESCRIPTIONS $\ldots \ldots \ldots \ldots \ldots \ldots \ldots \ldots, \mathbf{8}$

2.1 Introduction $\ldots \ldots \ldots \ldots \ldots \ldots \ldots \ldots \ldots \ldots \ldots$

2.2 The three-dimensional cloud chemistry model . . . . . . . 10

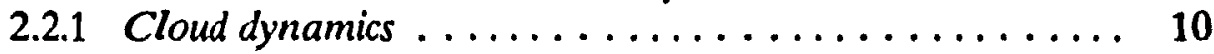

2.2 .2 Cloud chemisty ..................... 11

2.3 The Acid Deposition and Oxidant Model (ADOM) Scavenging Module ............................. 18

2.3.1 Cloud Physics Submodule . . . . . . . . . . . . . . 18

2.3.2 Aqueous-Phase Chemistry Submodule ............. 21

2.4 Common characteristics and important differences in the models ............................ 26

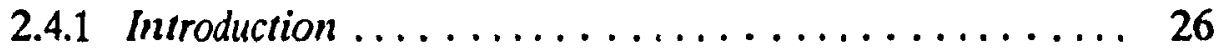

2.4.2 Domain and grid cell dimensions ............ 26

2.4 .3 Cloud formation ................... 29

2.4 .4 Modelled species . .................... 30

2.4.5 Scavenging of gaseous pollutants by the cloud water . . . . 31

2.4.6 Aerosol composition and scavenging .......... 33

2.4 .7 Aqueous-phase oxidation reactions ........... 35

Chapter 3 NUMERICAL EXPERIMENTS $\ldots \ldots \ldots \ldots \ldots \ldots \ldots \ldots, 36$

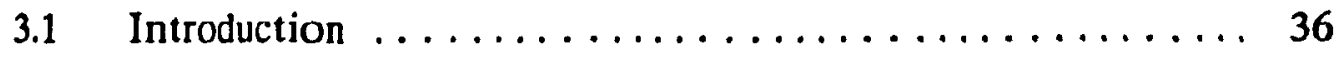

3.2 Three-dimensional Cloud Chemistry Model Simulation . . . . . . 37

3.2 .1 Cloud Dynamics Simulation . . . . . . . . . . . . 37

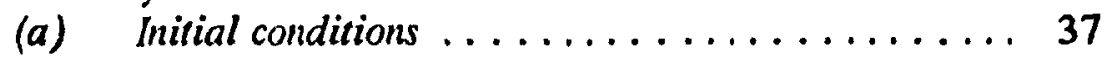

(b) Model results ................... 39 
3.2.2 Cloud Chemistry Simulation . . . . . . . . . . . . 43

(a) Initial conditions $\ldots \ldots \ldots \ldots \ldots \ldots \ldots \ldots 43$

(b) Model results .................... 46

3.3 ADOM Scavenging Module Simulation ........... 47

3.3.1 Model initialization .................. 47

3.3.2 ADOM clouds .................... 51

3.3.3 Model output .................... 55

Chapter 4 EVALUATION OF THE ADOM SCAVENGING MODULE ... 56

4.1 Introduction $\ldots \ldots \ldots \ldots \ldots \ldots \ldots \ldots \ldots \ldots \ldots \ldots$

4.2 Wet deposition of sulphate, nitrate and ammonium ions ..... 58

4.3 Rain acidity $(\mathrm{pH}) \ldots \ldots \ldots \ldots \ldots \ldots \ldots \ldots \ldots \ldots \ldots$

4.4 Wet deposition of hydrogen peroxide $\ldots \ldots \ldots \ldots \ldots \ldots \ldots 68$

4.5 Total and in-cloud oxidation $\ldots \ldots \ldots \ldots \ldots \ldots \ldots \ldots, 70$

4.6 Fractional acidity . . . . . . . . . . . . . . . . 75

4.7 Concentration of chemicals ................. 78

4.8 Comparison of model results for different ADOM clouds . . . . 81

Chapter 5 SUMMARY AND CONCLUSIONS $\ldots \ldots \ldots \ldots \ldots \ldots \ldots 84$

5.1 Summary ....................... 84

5.2 Conclusions $\ldots \ldots \ldots \ldots \ldots \ldots \ldots \ldots \ldots \ldots \ldots \ldots$

5.3 Suggestion for future studies $\ldots \ldots \ldots \ldots \ldots \ldots \ldots \ldots .89$

List of References $\ldots \ldots \ldots \ldots \ldots \ldots \ldots \ldots \ldots \ldots \ldots \ldots$ 


\section{LIST OF FIGURES}

Figure

Page

2.1 Idealization of Cloud System (Venkatram and Karamchandani, 1988) . . . . . . . . . . . . . . . . . . . . . . . . . . . . 19

2.2 Location of grid cells for ADOM simulations (Venkatram, 1986) . . . 27

3.1 Temperature and humidity profiles plotted on the tephigram ..... 38

3.2 Hodograph of the initial horizontal wind $\ldots \ldots \ldots \ldots$

3.3 Variation of the total cloud water content of CLOUD A with time ... 40

3.4 Variation of the total cloud water content of CLOUD B with time . . 40

3.5 Variation of the total rain water content of CLOUD A with time . . . 41

3.6 Variation of the total rain water content of CLOUD B with time . . . 41

3.7 Variation of the total precipitation rate of CLOUD A with time . . . . 42

3.8 Variation of the total precipitation rate of CLOUD B with time ... . 42

3.9 Vertical dimensions of the simulated clouds $\ldots \ldots \ldots \ldots \ldots \ldots$

4.1 Wet deposition of sulphate by CLOUD $A$ and corresponding ADOM clouds for different chemical environments . . . . . . . . . . . 59

4.2 Wet deposition of sulphate by CLOUD B and corresponding ADOM clouds for different chemical environments . . . . . . . . . . . . 59

4.3 Wet deposition of sulphate, nitrate and ammonium, expressed as a difference between wet-deposited amount predicted by the cloud chemistry model for CLOUD A and the corresponding ADOM cloud, divided by the cloud chemistry model prediction . . . . . . . . . 60

4.4 Wet deposition of sulphate, nitrate and ammonium, expressed as a difference between wet-deposited amount predicted by the cloud chemistry model for CLOUD B and the corresponding ADOM cloud, divided by the cloud chemistry model prediction ........... 60 
4.5 Wet deposition of nitrate by CLOUD A and corresponding ADOM clonds.

4.6 Wet deposition of nitrate by CLOUD B and corresponding ADOM

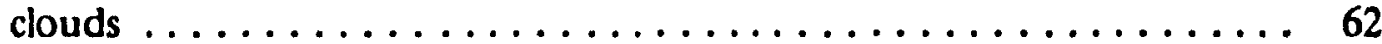

4.7 Wet deposition of ammonium by CLOUD $A$ and corresponding ADOM clouds .......................... 64

4.8 Wet deposition of ammonium by CLOUD B and corresponding ADOM clouds ............................. 64

4.9 Rain acidity for CLOUD A and corresponding ADOM clouds . . . . 67

4.10 Rain acidity for CLOUD B and corresponding ADOM clouds . . . . 67

4.11 Wet deposition of hydrogen peroxide by CLOUD A and corresponding

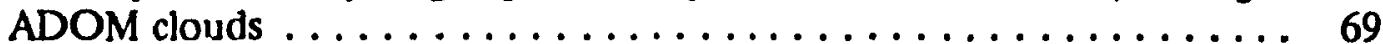

4.12 Wet deposition of hydrogen peroxide by CLOUD B and cnrresponding ADOM clouds . . . . . . . . . . . . . . . . . . . . . . . . 69

4.13 Total oxidation for CLOUD A and corresponding ADOM clouds . . . 71

4.14 Total oxidation for CLOUD B and corresponding ADOM clouds ... 71

4.15 In-cloud oxidation fraction for CLOUD $A$ and corresponding ADOM clouds

4.16 In-cloud oxidation fraction for CLOUD B and corresponding ADOM clouds

4.17 Fractional acidity for CLOUD A and corresponding ADOM clouds .. 77

4.18 Fractional acidity for CLOUD B and corresponding ADOM clouds .. 77

4.19 Difference between final and initial sulphate in the air for CLOUD A

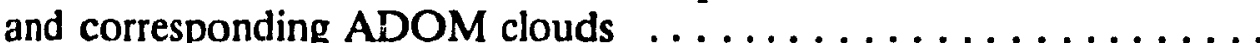

4.20 Difference between final and initial sulphate in the air for CLOUD B and corresponding ADOM clouds 


\section{LIST OF TABLES}

Table

Page

2.1 Species in the condensed aqueous-phase mechanism (Venkatram and Karamchandani, 1988) ....................... 22

22 Reactions describing the reversible mass transfer of soluble gases to cloud water (Venkatram and Karamchandani, 1988) . . . . . . . . . 23

2.3 Reactions describing the irreversible scavenging of aerosols by cloud water (Venkatram and Karamchandani, 1988) . . . . . . . . . . 24

2.4 Aqueous-phase oxidation-reduction reactions (Venkatram and Karamchandani, 1988) ..................... 25

2.5 ADOM vertical structure (Venkatram, 1986) $\ldots \ldots \ldots \ldots$

2.6 Reactions and solubility or equilibrium constants $\ldots \ldots \ldots \ldots \ldots$

3.1 Initial concentrations of chemicals, expressed in $\mu \mathrm{g} / \mathrm{m}^{3}$ for $\mathrm{SO}_{4}$ and in ppbv for all other species .................... 45

3.2 Initial concentrations of chemicals, expressed in $\mu \mathrm{g} / \mathrm{m}^{3}$ for $\mathrm{SO}_{4}$ and in ppbv for all other species .................... 46

3.3 Characteristics of simulated clouds $\ldots \ldots \ldots \ldots \ldots \ldots \ldots$ 


\section{Chapter 1}

\section{INTRODUCTION}

\subsection{Overview}

The problem of acid deposition is far from being completely understood, although during the last decade this problem has become the subject of an increasing number of studies. Acid deposition is a complicated phenomenon. Several related processes have to be analyzed: the emission of acidic precursors into the atmosphere, their transport, transformation by different chemical and physical processes, and finally deposition at the surface. Understanding of these processes and their relationship can be improved by modelling, which is why the so called LRTAP or long-range transport of atmospheric pollutant models have been developed.

The Acid Deposition and Oxidant Model, henceforth referred to as ADOM, developed by Environmental Research and Technology (ERT) and Meteorological and Environmental Planning (MEP) Company of Canad? (Venkatram et al., 1988) belongs to the class of LRTAP models. The Regional Acid Deposition Model (RADM) (Chang et al., 1987) belongs to the same class. STEM I (Carmichael and Peters, 1984) and STEM II (Carmichael et al., 1991), where STEM is the acronym for the Sulphur Transport Eulerian Model, are regional-scale transport, chemistry and 
deposition models. All these models adopted the Eulerian framework as being more appropriate to describe acid deposition phenomenon. The models are modular in structure.

Each process simulated by the LRTAP model (e.g. dry deposition, transport, gas-phase chemistry, cloud chemistry or wet scavenging) is in reality complex. However, because of computational constraints, the modules that simulate these processes often introduce substantial simplifications. It is extremely important to "reach an adequate compromise between thoroughness of treatment and realistic possibilities of application" (:rinarne and Cho, 1989).

One of the most important components of any acid deposition model is its cloud module as it is well established that aqueous-phase reactions in clouds are major contributors to atmospheric acidification. $A D O M$ includes two different modules for clouds: one describes stratus clouds and the other simulates convective clouds (Karamchandani and Venkatram, 1992). RADM uses a one-dimensional cloud module with a box aqueous-chemistry and scavenging model developed by Walcek and Taylor (1986). There are currently two versions of STEM II that Ciffer in their treatment of clovd properties (Carmichael et al., 1991). One version includes Advanced Scavenging Module (ASM) developed by Easter and Hales (1984), the second version incorporates a more realistic one-dimensional cloud model developed by Berkowitz et al. (1989). 
Among all the components of ADOM, the cloud module, describing cloud formation, pollutant scavenging, aqueous-phase chemistry and eventually wet deposition, seems to be subject to the biggest uncertainties. It has been already improved since the first report of $\mathrm{ADOM}$ but it is still an object of investigation. The recent version of the $A D O M$ cloud module is called also scavenging module (Venkatram and Karamchandani, 1988; Karamchandani and Venkatram, 1992) or wet scavenging module (Venkatram et al., 1988; Venkatram and Karamchandani, 1986). Throughout this study the ADOM cloud module will be referred to as scavenging module.

This module, like any other ADOM module, has to satisfy computer space and time requirements and consequently it can not be too elaborate, but still it must capture the main features of the phenomena to be described.

Evaluation of any model requires comparison of its predictions with observations. ADOM has been tested against data collected during the field study undertaken in 1981: Oxidation and Scavenging Characteristics of April Rains OSCAR (Easter et al., 1984). Recently, in 1990, another field program has been completed: Eulerian Model Evaluation Field Study - EMEFS (Hansen et al., 1991). The data collected during this program allow further evaluation of ADOM. But comparison of model predictions with observations is not straightforward in the case of a comprehensive acid deposition model. The observed and measured fields are 
subject to spatial and temporal variability that cause problems of accuracy and representativenes with the collected data. Another problem is the availability of an appropriate data set that satisfies the needs of the model. "Comparison of model predictions with observations is a formidable task because of the enormous data requirements of a comprehensive (ADOM) model. Data sets that can be considered adequate are not available now, nor are likely to be in the foreseeable future" (Venkatram et al., 1988).

\subsection{Purpose of this study}

The purpose of this study is the evaluation of the ADOM scavenging module. This evaluation will be done by model inter-comparisons.

The ultimate goal of any model is to simulate the behaviour of a chosen piece of the real observed world. So it seems obvious that the model should be evaluated by comparison of its predictions with measurements and not with another model's predictions. Far from denying this fundamental truth, this study proposes a complementary approach to model evaluation. A number of factors, presented below, seem to justify the need for this type of analysis when the huge, LRTAP models are considered.

These big models are built up from separate parts. Each part can introduce an uncertainty or inaccuracy in description which is difficult to evaluate. The final 
result, subject to testing against observations, is influenced by the whole computing sequence. As an example let's take the observed and model-predicted amount of wet deposition of a particular chemical species. Among initial data there is information about emission of chemical species which are the precursors of the wet-deposited pollutants. Before the amount of wet deposition of each species is predicted by the model, transport of emitted precursors, gas phase reactions, depletion between the source and the target, cloud development, in-cloud processes and finally the rain formation have to be described. How the accuracy of the description of these consecutive steps influence the final result is hard if not impossible to determine. All this considered it is evident that evaluation of individual ADOM modules in isolation is essential to improve the understanding of their efficiency, weaknesses and strong points.

Evaluation of a separate ADOM module requires specification of particular initial data. As each module simulates only a given aspect of described processes, the data should be representative for the actually modelled feature. In the case of the scavenging module, for instance, the emissions of precursors should be replaced by the concentration of the species in the air before the cloud develops. In addition, data that allow the simulation of the cloud within the observed area should be also available. Field programs, expensive and time consuming as they are, can not provide us with adequate data. As is the case for the entire ADOM where the full set of required data is rarely available, the specific data necessary to initialize the scavenging module are also difficult to acquire. The problems of their accuracy and 
representativeness are also significant.

Fortunately, there exists a model, the three-dimensional convective cloud chemistry model developed at McGill, which describes reasonably well the dynamics and chemistry of convective clouds (Tremblay and Leighton, 1986). The threedimensional cloud chemistry model is much more realistic than the ADOM scavenging module in simulating cloud formation: it includes fairly complex physics, dynamics and microphysics of both cloud and rain. It has been tested against data in diverse situations giving predictions close to the observations (Yau and Michaud, 1982; Yau and Macpherson, 1984; Tremblay, 1987; Leighton et al., 1990). It seems reasonable to use it as a generator of a surrogate data field. The role of these data sets is two-fold: they allow the initialization of the isolated portion of ADOM and then the comparison of its results with the cloud chemistry model results.

What are the advantages of proposed inter-model comparisons?

Inter-model comparisons evaluate the ADOM scavenging module in isolation. The results of simulations are not influenced by the behaviour of the other parts of ADOM. This kind of analysis can help understanding the properties of the scavenging module alone.

Actual data fields are not required and consequently no problem of data accuracy or representativeness exists. Simulations may be performed for as many 
initial data sets as we decide to generate and may characterise as many different environments as we choose to analyze. This approach enables more systematic studies. Comparisons of the output of the ADOM scavenging module with output from the cloud chemistry model for equivalent input conditions allows one to investigate more closely the limitations of the scavenging module and its performance in describing a variety of situations that can be easily simulated by the other model.

As the computational demands of the three-dimensional cloud chemistry model are much greater than those of the ADOM module it can by no means replace the scavenging module in ADOM. But it can constitute a laboratory that allows testing of the scavenging module performance in different situations. This will be done according to the following scheme:

- modify the chemistry of the existing three-dimensional cloud chemistry model in order that it be as similar as possible to the ADOM scavenging module;

- run the three-dimensional cloud chemistry model;

- generate a set of initial data giving the equivalent initial conditions for the ADOM scavenging module;

- run the ADOM scavenging module;

- compare the results;

- repeat four last points for another set of initial conditions;

- evaluate the scavenging module of ADOM. 


\section{Chapter 2}

\section{MODEL DESCRIPTIONS}

\subsection{Introduction}

The model used in this study to evaluate the scavenging module of ADOM is the three-dimensional convective cloud chemistry model developed by Tremblay and Leighton (1986). This model is actually a combination of two uncoupled numerical models: the cloud dynamics and cloud chemistry models. The cloud dynamics model describes cloud formation and it is run alone generating the date that can be subsequently used to initialize and run the cloud chemistry part of the model. This procedure is justified by the assumption that chemistry does not affect the dynamics and microphysics of the evolving cloud. The pertinent information about the two parts of the three-dimensional cloud chemistry model is given in section 2.2 . The formulas and values of coefficients and constants are specified in this section only if the equivalent expression is not utilised in ADOM. The remaining information, missing at this point, will be found in section 2.4 after the description of the ADOM scavenging module. 
The chemistry part of the ADOM scavenging module is decoupled from the part that describes cloud formation, as in the cloud chemistry model, although in ADOM the two parts are incorporated into the same model. Cloud formation and dissipation are described by the Raymond and Blyth (1986) mixing scheme. The chemistry is simulated by the aqueous-phase chemistry submodule. The description of the ADOM scavenging module follows in section 2.3.

Cloud dynamics and microphysics, simulated in the three-dimensional cloud chemistry model, is substantially different from the Raymond and Blyth mixing scheme. However, the chemistry approach applied in the cloud chemistry model is similar to that in ADOM. The similarity can be maximised by ensuring that equilibrium constants and reaction rate constants in the ADOM scavenging module and the cloud chemistry model match. The list of common constants used in models is presented in section 2.4. That section focuses on differences in the models and on methods of minimizing the discrepancies. 


\subsection{The three-dimensional cloud chemistry model}

\subsubsection{Cloud dynamics}

The cloud dynamics model was developed by Steiner (1973) and Yau (1981). It was originally designed to describe a single isolated convective cloud. Subsequently it has been extensively and successfully used to simulate cumulus ensembles (Yau and Michaud, 1982), hailstorms (Yau and Macpherson, 1984) and rainbands (Leighton et al., 1990).

The single-cloud version of the model simulates in three dimensions the development of a moderate-sized precipitating cumulus cloud starting from an initial humidity impulse.

It needs as input data the domain and grid size description. Temperature and humidity profiles, horizontal wind distribution with height, and pressure at the surface must be specified as well. Initially all these fields are assumed to be horizontally uniform in the domain of integration. The properties of the initial humidity impulse that triggers cloud and rain formation have to be defined. The autoconversion threshold has to be chosen and a decision whether the diffusion of rain will be permitted must also be taken. Having specified all these parameters as well as time related data: simulation time, time step and frequency of recording output data on 
the tape, the model can be run. It gives at each time step the necessary input data to run the second part of the model, its chemistry component. These data include three dime:sional fields of wind, eddy diffusion coefficients, atmospheric water (water vapour, cloud and rain water) and information about occurrence of condensation and evaporation in each grid box.

The results of a dynamics run may be stored. As a consequence it is possible to perform several chemistry runs for the same dynamical situation, what means that the cloud, simulated by the cloud dynamics model, can subsequently grow in different chemical environments. The same cloud may dissolve greater or smaller amounts of various chemical species, oxidation reactions within the cloud may be more or less significant, and finally the wet deposition and redistribution of chemical species in the atmosphere will also vary according to chemical properties of the environment.

\subsubsection{Cloud chemistry}

The cloud chemistry model has to be initialized in a manner consistent with the cloud dynamics model. The time and space resolution have to be the same, and the initial temperature profile, and pressure at the surface have to be common to the both models. The additional data that are required to initialize the cloud chemistry model are the concentration profiles of the modelled species. 
Chemical species included in the model are as follows:

(1) sulphur dioxide, (2) ozone, (3) hydrogen peroxide, (4) nitric acid, (5) ammonia, (6) carbon dioxide and (7) sulphate aerosol.

Each modelled chemical may exist either in cloud, air, or rain. The distribution between different phases depends on the species.

All the above mentioned species and their most relevant properties are described briefly below. A detailed presentation of the chemistry involved in this model can be found in Tremblay and Leighton (1986), Pitre (1986) or Giles (1987).

(1) Sulphur dioxide

Sulphur dioxide dissolves in cloud and rain water. Its dissolution is $\mathrm{pH}$ dependent. In the liquid phase dissolved sulphur dioxide dissociates and rapidly establishes aqueous-phase equilibria. The following equations describe the dissolution of gaseous $\mathrm{SO}_{2}$ and aqueous-phase equilibria:

$$
\begin{aligned}
& \mathrm{SO}_{2}(\mathrm{~g}) \leftrightarrow \mathrm{SO}_{2}(\mathrm{aq}) \\
& \mathrm{SO}_{2}(\mathrm{aq}) \leftrightarrow \mathrm{HSO}_{3}+\mathrm{H}^{+} \\
& \mathrm{HSO}_{3}+\mathrm{SO}_{3}{ }^{-}+\mathrm{H}^{+}
\end{aligned}
$$


Aqueous sulphur dioxide $\left(\mathrm{SO}_{2}(\mathrm{aq})\right)$, bisulphite $\left(\mathrm{HSO}_{3}{ }^{\circ}\right)$, and sulphite $\left(\mathrm{SO}_{3}{ }^{-}\right)$ are together referred as S(IV). The aqueous concentration of S(IV) is related to the gaseous concentration of sulphur dioxide expressed by its partial pressure $\left(\mathrm{PsO}_{2}\right)$ by the effective Henry's Law constant $\left(H_{S(M)}\right)$ that includes the totality of dissociation products (Schwartz, 1986):

$$
\begin{gathered}
{[S(I V)]=H_{S(I V)} \times p_{S O_{2}}} \\
H_{S(I V)}=H_{S O_{2}}\left(1+\frac{K_{1 S}}{\left[H^{+}\right]}+\frac{\left.K_{1 S} K_{2 S}\right)}{\left[H^{+}\right]^{2}}\right.
\end{gathered}
$$

$\mathrm{H}_{\mathrm{SO}_{2}}, \mathrm{~K}_{1 \mathrm{~s}}$ and $\mathrm{K}_{2 \mathrm{~s}}$ are respectively the Henry's Law and dissociation constants describing aqueous-phase equilibria represented by the three equations above.

Solubility equilibrium is assumed for the sulphur dioxide inside the cloud, because the characteristic time constant for in-cloud scavenging is small, less than 10 s (Tremblay and Leighton, 1986). Washout is assumed to occur at equilibrium (Tremblay and Leighton, 1986). This is a satisfactory approximation for sulphur dioxide scavenging in acidic situations, characterised by $\mathrm{pH}$ below 5 , according to criterion suggested by Hill and Adamowicz (1977).

S(IV) can be oxidized in the presence of hydrogen peroxide and ozone to $\mathrm{S}(\mathrm{VI})$. This aqueous-phase oxidation is described by rate expression:

$$
\frac{d}{d t}[S(V I)]=R_{i} \times[i] \times[S(I V)] \quad i=\mathrm{H}_{2} \mathrm{O}_{2}, \mathrm{O}_{3}
$$




\section{(2) Ozone and (6) carbon dioxide}

Ozone is an important oxidant. The presence of carbon dioxide in cloud and rain water influences its acidity.

The air concentrations of ozone and carbon dioxide are assumed to be constant during the simulation. Although oxidation processes deplete ozone, this depletion is insignificant if compared with the total ozone present in the cloud environment. Both gases are in equilibrium with the aqueous-phase. This equilibrium is expressed by appropriate Henry's Law constants. For carbon dioxide this is an effective Henry's Law constant as this species dissociates in water.

(3) Hydrogen peroxide

The importance of hydrogen peroxide in cloud chemistry models is due to its ability to oxidize S(IV).

The partition of hydrogen peroxide between gas and aqueous phases is determined by its Henry's Law constant. The scavenging of hydrogen peroxide by the rain is assumed to be an irreversible process (Tremblay and Leighton, 1986). It is described in the model by the same formulas as presented below for nitric acid and ammonia. 
(4) Nitric acid and (5) a mmonia

Nitric acid and ammonia are both non-reactive species in the model which means that no source or sink of total nitrate or ammonia exist and their concentration can not be changed by any chemical reaction, only by microphysical or dynamical processes. Both are highly soluble gases and in the presence of cloud are assumed to dissolve completely and become entirely ionized.

When rain forms, nitric acid and ammonia are transferred to rain water. Falling rain scavenges nitric acid, ammonia, and hydrogen peroxide as mentioned above, and the process is assumed to be irreversible. This seems to be a satisfactory approximation for the considered acidic situations (Tremblay and Leighton, 1986). The idea of a washout coefficient $\left(\Lambda_{1, a}\right)$ is utilized to describe the mechanism of scavenging, following the Levine and Schwartz (1982) formulation:

$$
\frac{d Q_{1, I}}{d t}=\Lambda_{i, \text { ar }} Q_{i, \mathrm{a}} \quad i=\mathrm{HNO}_{3}, N \mathrm{NH}_{3}, \mathrm{H}_{2} \mathrm{O}_{2}
$$

where a stands for air, $r$ for rain, and $Q_{i, a}$ or $Q_{i, r}$ is concentration of species $i$ respectively in the air or rain .

The relation between rain water content $\left(Q_{1}\right)$ and washout coefficient $\left(\Lambda_{1, e r}\right)$ suggested by Tremblay and Leighton (1986) is used: 


$$
\Lambda_{H N O_{3}, a I}=4.98 \times 10^{-5}+4.02 \times 10^{-4} Q_{x}
$$

where rain water content is in $\mathrm{gm}^{-3}$ and washout coefficient in $\mathrm{s}^{-1}$.

$$
\begin{aligned}
& \Lambda_{N_{3}, a r}=1.6 \Lambda_{H N O_{3}, a r} \\
& \Lambda_{H_{2} O_{2}, a r}=1.1 \Lambda_{H N O_{3}, a r}
\end{aligned}
$$

(7) Sulphate aerosol

Sulphate aerosol in the model is assumed to be composed of sulphuric acid $\left(\mathrm{H}_{2} \mathrm{SO}_{4}\right)$ and ammonium sulphate $\left(\left(\mathrm{NH}_{4}\right)_{2} \mathrm{SO}_{4}\right)$. The proportion of these two types of aerosol is constant in the model. For the simulations described in this thesis the ratio of sulphuric acid to the total amount of sulphate within the aerosol expressed as molar concentration is taken to be $\mathbf{0 . 6 2}$.

Sulphate aerosol exists in cloud water as the result of two processes: nucleation and $\mathrm{SO}_{2}$ oxidation. Nucleation is modelled as follows. If in a given grid box at cloud base condensation occurs for the first time, then a constant fraction of aerosol present in the and box is nucleated. This fraction, defined as the nucleation efficiency, is taken to be 0.5 in the model. The same fraction is used to describe the nucleation at cloud base associated with condensation that does not occur for the first time but is related to an updraft. The nucleation efficiency then depicts the fraction 
of sulphate mass flux at cloud base that is transferred to the cloud water by nucleation.

Sulphate enters rain water by microphysical processes (autoconversion and cloud collection), by washout, and it can be also formed within rain water as a result of $\mathrm{SO}_{2}$ oxidation.

The microphysical processes are formulated in the cloud dynamics model after Kessler (1969). The washout coefficient is calculated following Scott (1978):

$$
\Lambda_{S O_{1}, \mathrm{ar}}=5.2 \times 10^{-3} \epsilon_{8} Q_{S O_{4}, \mathrm{a}}^{0.875}
$$

where $c_{8}$ is an average collection efficiency taken to be 0.1 in the model and $Q_{s o 4, a}$ is the sulphate concentration in the air. Oxidation of sulphur in rain is described in the same way as in cloud. 


\subsection{The Acid Deposition and Oxidant Model (ADOM) Scavenging Module}

The ADOM scavenging module consists of two major components: the cloud physics submodule and the aqueous-phase chemistry submodule.

\subsubsection{Cloud Physics Submodule}

This module describes the formation and dissipation of cloud in the model. The formation takes place at the very beginning of the simulation time step, the dissipation at the very end. During the cloud lifetime its microphysics does not change, only scavenging and aqueous-phase chemistry occur. It is assumed in the module that only some fraction of the cloud volume, that will be referred to subsequently as the active cloud fraction, is invo'ved in the mixing of air parcels that have undergone vertical motion. Scavenging and aqueous-phase chemistry occur only in the active region of the cloud. If an ADOM cloud precipitates, a portion of the chemical species in the cloud water in the active region is wet-deposited and the remaining portion is redistributed in the air throughout the grid volume. The cloud system as modelled is presented in figure 2.1. 


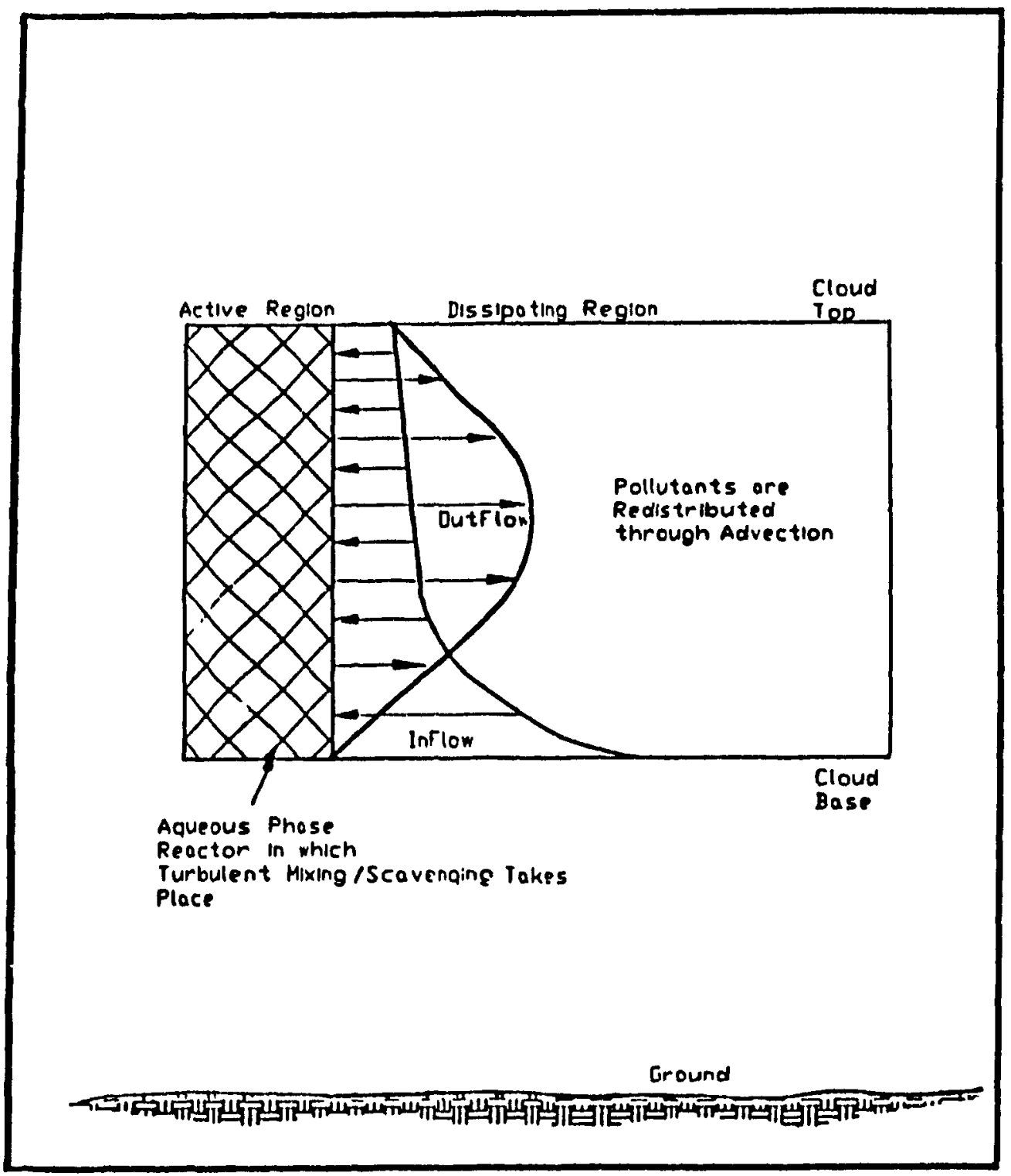

Figure 2.1 Idealization of Cloud System (Venkatram and Karamchandani, 1988) 
The active cloud fraction is described by application of the Raymond and Blyth (1986) mixing scheme. It requires as input data temperature, pressure, and relative humidity profiles, cloud base location and optionally cloud top location. Its output gives information about:

- air fluxes into and out of the active region at various levels of the cloud; that information allows the determination of concentration of the chemical species within the cloudy region;

- properties of the cloud formed by mixing; these properties include cloud base and top location, liquid water content and temperature within the cloud.

At the beginning of the simulation time step, which is one hour, the cloud is created and its properties are determined. The aqueous-phase chemistry takes place during the whole one hour, after which cloud eventually precipitates and redistribution of remaining species occurs. But this is not always true.

It is very important to stress here that the precipitation rate value is not found by the model itself, but is provided as initial data. It may happen that the simulated cloud, whose properties deptnd on the temperature and humidity profiles, assumed location of cloud base and top, cloud cover and active cloud fraction can not "produce" a sufficient amount of rain (the rain efficiency is an internal model parameter which is equal to 0.75 ). There then exists the possibility of simulating up to six clouds sequentially during the simulation time step. For each cloud the same 
principal rules apply: cloud properties are determined as soon as cloud forms, then aqueous-phase chemistry takes place for the full cloud lifetime and, at the end, the cloud precipitates and dissipates. The wet deposition for the simulation time step is the sum of the amounts wet-deposited by each cloud. Each of the individual clouds that occur within a single simulation time step is referred in the model as a cycle. There can be from one to six cycles during the one hour simulation time step, depending on the values of the initial data.

\subsubsection{Aqueous-Phase Chemistry Submodule}

Once the cloud is formed, its composition is modified by scavenging of gaseous and particulate species and reactions that take place in cloud water. The detailed aqueous-phase chemistry description in the first formulation of ADOM consisted of as many as 111 reactions between 47 species (Young and Lurmann, 1984). The version that we used has simplified aqueous-phase chemistry (Venkatram and Karamchandani, 1988) and incorporates only 13 species in the aqueous-phase, 12 species in the gas-phase and 25 reactions between them. The list of modelled gaseous, particulate and aqueous species is presented in table 2.1. The reactions between species can be divided in three groups:

- reversible mass transfer processes;

- irreversible scavenging of aerosols;

- aqueous-phase oxidation-reduction reactions. 
Table 2.1 Species in the condensed aqueous-phase mechanism (Venkatram and Karamchandani, 1988)

\begin{tabular}{||ll|ll||}
\hline \multicolumn{2}{|c|}{ Gas or Particulate Species } & \multicolumn{2}{c|}{ Aqueous Species } \\
\hline 1. Sulphur Dioxide & $\mathrm{SO}_{2}(\mathrm{~g})$ & Bisulphite & $\mathrm{HSO}_{3}(\mathrm{aq})$ \\
2. Ozone & $\mathrm{O}_{3}(\mathrm{~g})$ & Ozone & $\mathrm{O}_{3}(\mathrm{aq})$ \\
3. Hydrogen Peroxide & $\mathrm{H}_{2} \mathrm{O}_{2}(\mathrm{~g})$ & Hydrogen Peroxide & $\mathrm{H}_{2} \mathrm{O}_{2}(\mathrm{aq})$ \\
4. Nitric Acid & $\mathrm{HNO}_{3}(\mathrm{~g})$ & Nitrate & $\mathrm{NO}_{3}^{3}(\mathrm{aq})$ \\
5. Ammonia & $\mathrm{NH}_{3}(\mathrm{~g})$ & Ammonium & $\mathrm{NH}_{4}^{+}(\mathrm{aq})$ \\
6. Carbon Dioxide (Constant) & $\mathrm{CO}_{2}(\mathrm{~g})$ & Bicarbonate & $\mathrm{HCO}_{3}(\mathrm{aq})$ \\
7. Sulphuric Acid Aerosol & $\mathrm{H}_{2} \mathrm{SO}_{4}(\mathrm{p})$ & Sulphate & $\mathrm{SO}_{4}^{-(a q)}$ \\
8. Ammonium Sulphate Aerosol & $\left.\mathrm{NH}_{4}\right)_{2} \mathrm{SO}_{4}(\mathrm{p})$ & Hydrogen Ion & $\mathrm{H}^{+}(\mathrm{aq})$ \\
9. Ammonium Bisulphate Aerosol $\mathrm{NH}_{4} \mathrm{HSO}(\mathrm{p})$ & Hydroxide Ion & $\mathrm{OH}^{-}(\mathrm{aq})$ \\
10. Ammonium Nitrate Aerosol & $\mathrm{NH}_{4} \mathrm{NO}_{3}(\mathrm{p})$ & Water (Constant) & $\mathrm{H}_{2} \mathrm{O}(\mathrm{aq})$ \\
11. Soil Dust Aerosol & $\left.\mathrm{DUST}^{2}\right)$ & Cations & CAT1(aq) \\
12. Organic Peroxide & $\mathrm{ROOH}(\mathrm{p})$ & Organic Peroxide & $\mathrm{ROOH}(\mathrm{aq})$ \\
& & Iron \& Manganese & FEMN(aq) \\
\hline
\end{tabular}

All the reactions are specified in tables $2.2,2.3$, and 2.4. A more detailed description of model reaction: follow.

Reversible mass transfer of soluble gases to cloud water (table 2.2) is described as separate forward and backward reactions. The forward mass transfer rates are estimated from collision theory and sticking coefficients. The Fuchs and Sutugin (1971) formula is used to compute the loss rate of a gaseous pollutant for a single cloud droplet of given diameter. Then the total loss rate to a mixture of drops is calculated assuming a mono-dispersed distribution with a diameter of $10 \mu \mathrm{m}$. The value of sticking coefficient is assumed to be $10^{-3}$. The backward mass transfer rate is defined by forward rate and the appropriate Henry's Law constant. 
Table 2.2 Reactions describing the reversible mass transfer of soluble gases to cloud water (Venkatram and Karamchandani, 1988)

\begin{tabular}{|c|c|}
\hline 1. $\mathrm{SO}_{2}(\mathrm{~g})$ & $>\mathrm{B}_{1} \times \mathrm{HSO}_{3}(\mathrm{aq})+\mathrm{B} \times \mathrm{H}^{+}(\mathrm{aq})$ \\
\hline 2. $\mathrm{HSO}_{3}(\mathrm{aq})+\mathrm{H}^{+}(\mathrm{aq})$ & $\ldots$ \\
\hline 3. $\mathrm{HNO}_{3}(\mathrm{~g})$ & $->B 1 \times \mathrm{NO}_{3}^{-}(\mathrm{aq})+\mathrm{B} \times \mathrm{H}^{+}(\mathrm{aq})$ \\
\hline 4. $\mathrm{NO}_{3}(\mathrm{aq})+\mathrm{H}^{+}(\mathrm{aq})$ & $\ldots \quad \mathrm{B} 2 \times \mathrm{HNO}_{3}(\mathrm{~g})$ \\
\hline 5. $\mathrm{H}_{2} \mathrm{O}_{2}(\mathrm{~g})$ & $\ldots>\mathrm{B}_{1} \times \mathrm{H}_{2} \mathrm{O}_{2}(\mathrm{aq})$ \\
\hline 6. $\mathrm{H}_{2} \mathrm{O}_{2}(\mathrm{aq})$ & $\ldots>\quad B 2 \times \mathrm{H}_{2} \mathrm{O}_{2}(\mathrm{~g})$ \\
\hline 7. $\mathrm{ROOH}(\mathrm{g})$ & B1 x ROOH(aq) \\
\hline 8. $\mathrm{ROOH}(\mathrm{aq})$ & B2 x ROOH(g) \\
\hline 9. $\mathrm{NH}_{3}(\mathrm{~g})$ & $\ldots>\mathrm{B}_{1} \times \mathrm{NH}_{4}^{+}(\mathrm{aq})+\mathrm{B} 1 \times \mathrm{OH}^{-}(\mathrm{aq})$ \\
\hline 10. $\mathrm{NH}_{4}^{+}(\mathrm{aq})+\mathrm{OH}^{-}(\mathrm{aq})$ & $\mathrm{B} 2 \times \mathrm{NH}_{3}(\mathrm{~g})$ \\
\hline 11. $\mathrm{O}_{3}(\mathrm{~g})$ & $\mathrm{B} 1 \times \mathrm{O}_{3}(\mathrm{aq})$ \\
\hline 12. $O_{3}(\mathrm{aq})$ & 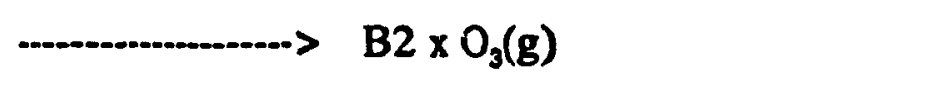 \\
\hline 13. $\mathrm{CO}_{2}(\mathrm{~g})$ & $\ldots \mathrm{B} 1 \times \mathrm{HCO}_{3}^{-}(\mathrm{aq})+\mathrm{B} 1 \times \mathrm{H}^{+}(\mathrm{aq})$ \\
\hline 14. $\mathrm{HCO}_{3}^{-}(\mathrm{aq})+\mathrm{H}^{+}(\mathrm{aq})$ & $\mathrm{B} 2 \times \mathrm{CO}_{2}(\mathrm{~g})$ \\
\hline
\end{tabular}

Coefficients:

$$
\begin{aligned}
& \text { B1 }=1 / \text { LRT } \\
& \text { B2 }=\text { LRT } \\
& \text { L = Liquid water volumetric fraction in air } \\
& R=\text { Universal gas constant } \\
& T=\text { Temperature }
\end{aligned}
$$


Irreversible scavenging of aerosols occurs as soon as cloud is formed. It is assumed that all the aerosols are available as cloud condensation nuclei. This means that all aerosols within the active region of the cloud are immediately found in the cloud water, nothing being left in the interstitial air. Table 2.3 lists the pertinent reactions.

Table 2.3 Reactions describing the irreversible scavenging of aerosols by cloud water (Venkatram and Karamchandani, 1988)

\begin{tabular}{|c|c|c|}
\hline 1. $\mathrm{H}_{2} \mathrm{SO}_{4}(\mathrm{p})$ & $\ldots \ldots$ & $\mathrm{B} 1 \times \mathrm{SO}_{4}^{-}(\mathrm{aq})+\mathrm{B}^{-} \times \mathrm{H}^{+}(\mathrm{aq})$ \\
\hline 2. $\mathrm{NH}_{4} \mathrm{HSO}_{4}(\mathrm{p})$ & $\ldots . . . . . . .>>$ & $\mathrm{B} 1 \times \mathrm{SO}_{4}=(\mathrm{aq})+\mathrm{B} 1 \times \mathrm{H}^{+}(\mathrm{aq})+\mathrm{B} 1 \times \mathrm{NH}_{4}^{+}(\mathrm{aq})$ \\
\hline 3. $\left(\mathrm{NH}_{4}\right)_{2} \mathrm{SO}_{4}(\mathrm{p})$ & -...----.> & $\mathrm{B} 1 \times \mathrm{SO}_{4}{ }^{-}(\mathrm{aq})+\mathrm{B}^{-} \times \mathrm{NH}_{4}{ }^{+}(\mathrm{aq})$ \\
\hline 4. $\mathrm{NH}_{4} \mathrm{NO}_{3}(\mathrm{p})$ & ...........> & $\mathrm{B} 1 \times \mathrm{NO}_{3}(\mathrm{aq})+\mathrm{B} 1 \times \mathrm{NH}_{4}^{+}(\mathrm{aq})$ \\
\hline 5. DUST(p) & $\ldots$ & $\mathrm{B} 5 \times \mathrm{FEMN}(\mathrm{aq})+\mathrm{B} 4 \times \mathrm{HCO}_{3}(\mathrm{aq})+\mathrm{B} 4 \times \mathrm{CAT} 1(\mathrm{aq})$ \\
\hline
\end{tabular}

Coefficients:

$$
\begin{aligned}
& \text { B1 }=1 / \text { LRT } \\
& \text { B3 }=2 / \text { LRT } \\
& \text { B4 }=.25 / \text { RRT } \\
& \text { B5 }=.05 / \text { LRT } \\
& \text { L = Liquid water volumetric fraction in air } \\
& \text { R }=\text { Universal gas constant } \\
& T=\text { Temperature }
\end{aligned}
$$

Aqueous-phase oxidation-reduction reactions describe four pathways for the oxidation of $S(I V)$ to $S(V I)$. The oxidizing factors are: ozone, hydrogen peroxide, organic peroxides, and oxygen in the presence of iron and manganese. In table 2.4 reactions, rate constants at $25^{\circ} \mathrm{C}$, and activation energies are presented. 
Table 2.4 Aqueous-phase oxidation-reduction reactions (Venkatram and Karamchandani, 1988)

\begin{tabular}{|c|c|c|}
\hline Stoichiometry & Rate Expression ( $\left.\mathrm{M} \mathrm{s}^{-1} @ 25^{\circ} \mathrm{C}\right)$ & $\begin{array}{l}\text { Activation } \\
\text { Energy } \\
\left.\text { (kcal mol }^{-1}\right)\end{array}$ \\
\hline 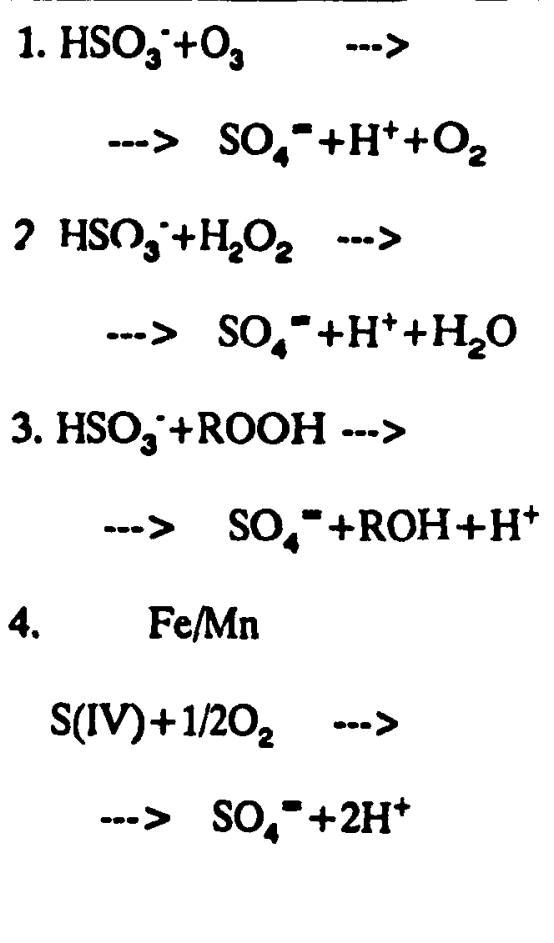 & $\begin{array}{l}8.03 \times 10^{7}\left[\mathrm{H}^{+}\right]\left[\mathrm{H}_{2} \mathrm{O}_{2}\right]\left[\mathrm{HSO}_{3}{ }^{-}\right] \\
\left\{1.82 \times 10^{7}\left[\mathrm{H}^{+}\right]+300.3\right\}[\mathrm{ROOH}]\left[\mathrm{HSO}_{3}\right] \\
4.17 \times 10^{7}\left[\mathrm{Fe}^{3+}\right]\left[\mathrm{Mn}^{2+}\right][\mathrm{S}(\mathrm{IV})]\left[\mathrm{H}^{+}\right]^{-0.74} \\
\mathrm{pH}<4.2 \\
2.81 \times 10^{13}\left[\mathrm{Fe}^{3+}\right]\left[\mathrm{Mn}^{2+}\right][\mathrm{S}(\mathrm{IV})]\left[\mathrm{H}^{+}\right]^{0.87} \\
\mathrm{pH} \geq 4.2\end{array}$ & 16.8 \\
\hline
\end{tabular}




\subsection{Common characteristics and important differences in the models}

\subsubsection{Introduction}

Both the scavenging module of ADOM and the cloud chemistry model are Eulerian models solving the mass conservation equations for pollutants. Pollutants, initially in the air, are scavenged by developing cloud, participate in chemical reactions in cloud water and are finally wet-deposited if the cloud precipitates. This general scheme is common to both models, although its realisation within the models is in general different.

The scavenging module can be run and studied independently of other ADOM modules. The information that it normally receives from other modules can be specified as initial data. Therefore comparisons can be performed between the scavenging module alone and the three-dimensional convective cloud chemistry model.

\subsubsection{Domain and grid cell dimensions}

The scavenging module can be run as an independent part of $A D O M$, but it still must satisfy some compatibility requirements. The domain and grid system is fixed for ADOM and they must be the same for each module. 
ADOM as a regional model is designed to analyze the problem of the air pollution on a larger scale than the cloud chemistry model. The application of the ADOM to the North American continent utilises a grid formed by 33 by 33 cells with grid spacing of $127 \mathrm{~km}$ as shown in figure $\mathbf{2 . 2}$

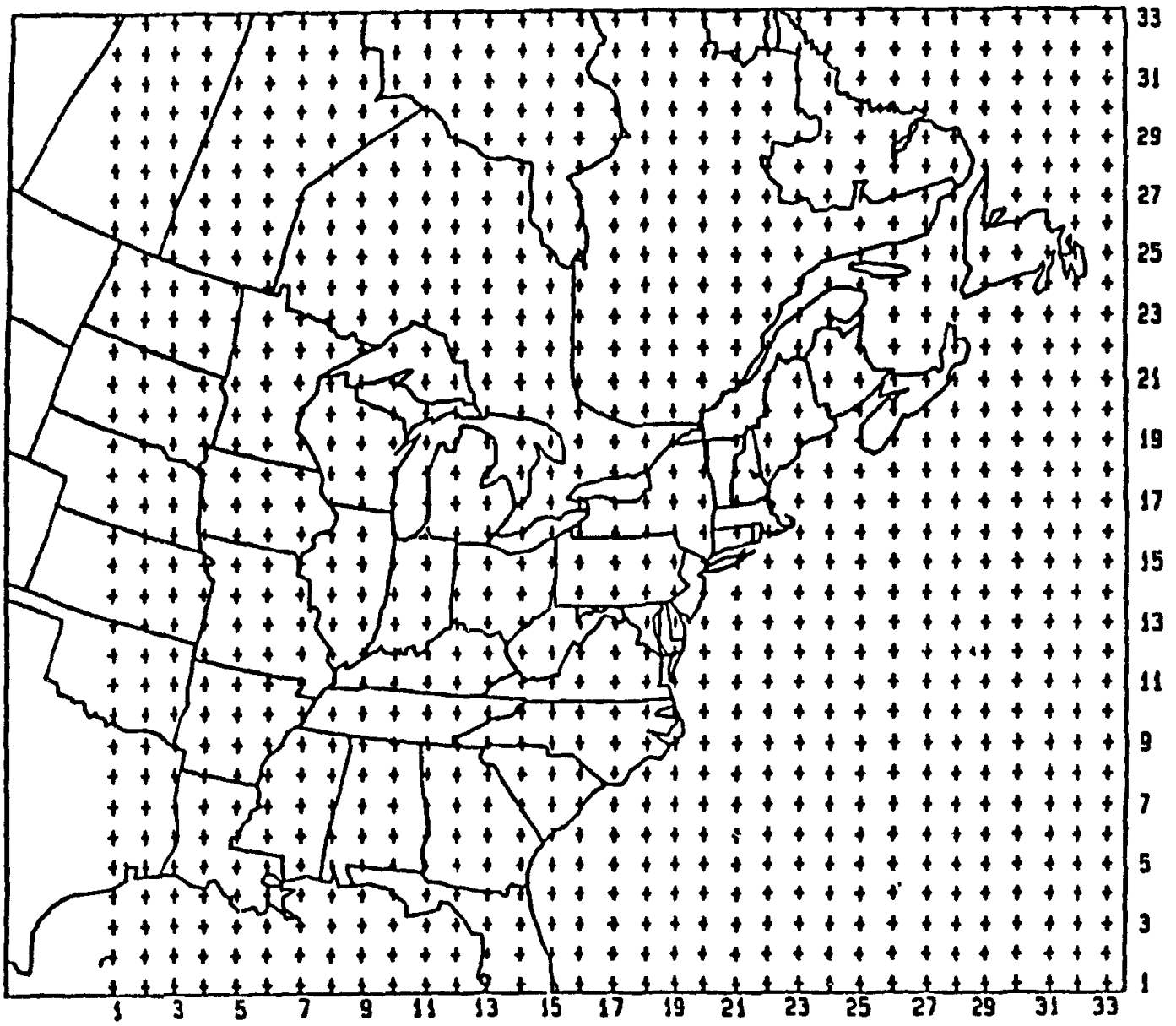

Figure 2.2 Location of grid cells for ADOM simulations (Venkatram, 1986) 
The vertical extent of the modelled domain is $10 \mathrm{~km}$ and it contains 12 levels. The vertical resolution is non uniform. The vertical structure of the grid "is based on a three-parameter logarithmic transformation " (Venkatram, 1986) and is shown in table 2.5. The ADOM grid "is designed to resolve the higher concentration gradients in the boundary layer" (Venkatram et al., 1988). The vertiral spacing, and particularly the large distances between higher ADOM layers, is an important factor for the model comparison. Its consequences will be discussed in more detail in the next chapter.

Table 2.5 ADOM vertical structure (Venkatram, 1986)

\begin{tabular}{|c|c|}
\hline Cell no. & Cell face top height $(\mathrm{m})$ \\
\hline 1 & 56.2 \\
2 & 135.8 \\
3 & 250.7 \\
4 & 416.3 \\
5 & 655.3 \\
6 & 1000.0 \\
7 & 1497.2 \\
8 & 2214.5 \\
9 & 3249.2 \\
10 & 4741.6 \\
11 & 6894.5 \\
12 & 10000.0 \\
\hline
\end{tabular}


The cloud chemistry model, applied in this study, is designed to simulate a single convective cloud. It utilises a regular vertical and horizontal grid to solve the model differential equations. Vertical and horizontal resolution may be chosen. In the simulations to be described, the model was run with a vertical resolution of $0.3 \mathrm{~km}$ and vertical extent of $6.9 \mathrm{~km}$, giving 23 levels. Horizontal resolution was $0.375 \mathrm{~km}$ and horizontal extent $12 \mathrm{~km}$, in both $\mathrm{x}$ and $\mathrm{y}$ directions, which gives $32 \times 32$ giid cells.

Model domains are not the same. One ADOM grid square $\left(127 \times 127 \mathrm{~km}^{2}\right)$ may be filled with many (about 112) cloud chemistry model domains. In order to facilitate the comparison of model results the ADOM scavenging module output was normalized to the cloud chemistry model domain.

\subsubsection{Cloud formation}

Cloud formation is described by the cloud dynamics part of the cloud chemistry model and by the Raymond and Blyth model in ADOM.

The cloud simulated by the cloud dynamics model is a three-dimensional object evolving in time. The chemistry part of cloud chemistry model reads at each time step the stored output from the cloud dynamics model. Subsequently it uses those data (winds, eddy diffusion coefficients, cloud water content, rain water content and records of the occurrence of condensation and evaporation of cloud and rain) 
to determine how the chemical concentration changes as a result of microphysical and chemical processes.

The cloud simulated by the ADOM scavenging module is one dimensional. The dynamics and microphysics of the ADOM cloud do not change during the simulation time step, so the concentration of chemicals change only as the resı!', of chemical processes. The active part of the cloud constitutes a homogeneous medium in which scavenging and chemical reactions take place. Only at the very end of the simulation time step does precipitation occur and mixing redistribute the chemicals throughout the entire volume of the cloudy region.

\subsubsection{Modelled species}

ADOM contains species, not included in the cloud chemistry model, that may influence the chemistry of the precipitation. These extra species exist both in the gas and aqueous phases. In the gas phase these are: ammonium bisulphate, ammonium nitrate and soil dust all present as aerosols, and organic peroxide as gas. Organic peroxide is present also in the aqueous phase. Soil dust that is transferred to the cloud water is converted to iron, manganese bicarbonate and cations (see table 2.3).

In order to evaluate the significance of these species sensitivity tests were performed. The ADOM scavenging module output values for two runs were 
compared. In the first run all species have realistic, non-zero initial concentrations. In the second run only species that exist in both models were assigned initial non-zero concentrations, which were the same as in the first run. All elements of output, that will be subsequently analyzed for model evaluation purposes, were compared. The discrepancies were found to be unimportant. The biggest difference was observed for the wet-deposited amount of sulphur dioxide, which was sometimes close to $5 \%$. The differences in $\mathrm{pH}$, in-cloud oxidation fraction, or in wet-deposited amount of other species common to beth models were generally below $1 \%$. Because of the minor influence of species which are not present in the cloud chemistry model, their concentrations can be set equal to zero in ADOM in the inter-comparison experiments.

\subsubsection{Scavenging of gaseous pollutants by the cloud water}

The scavenging of gases by cloud droplets is described in a different way in both models.

Two of the six gaseous species described by the cloud chemistry model, namely nitric acid vapour and ammonia gas, are assumed to dissolve completely in the cloud water. So nothing is left in the interstitial air, all is in cloud water. Two, hydrogen peroxide and ozone, are dissolved without dissociation according to the value of their respective Henry's Law constants. The last two, sulphur dioxide and carbon dioxide 
dissolve and then dissociate in the cloud water. Again the relative amounts of species in cloud water and in the air are determined according to the value of their respective Henry's Law constants, this time jointly with equilibrium constants for dissociation.

In ADOM, reversible mass transfer processes between gaseous and aqueous phases are expressed as separate forward and backward reactions as pointed out in section 2.3.2. The forward mass transfer is described within the context of collision theory, the backward mass transfer coefficients are expressed by the Henry's Law constants together with forward rates. This is not the same description as for the cloud chemistry model where aqueous-phase equilibrium is assumed for all species dissolved in cloud water. But since the time constant for the establishment of equilibrium is sma!! compared to the cloud lifetime the different approaches will not generate significantly different results.

All constants involved in the chemistry part of models can however have exactly the same values. The constants in the cloud chemistry model were set equal to the constants used in the ADOM code. The list of solubility and equilibrium constants used in both models is presented in table 2.6. The second dissociation of the aqueous sulphur dioxide (described by constant $\mathrm{K}_{2 \mathrm{~S}}$ ) is excluded from the cloud chemistry model as it is not considered in ADOM, the value of constant $K_{2 s}$ is taken to be zero. 
Table 2.6 Reactions and solubility or equilibrium constants

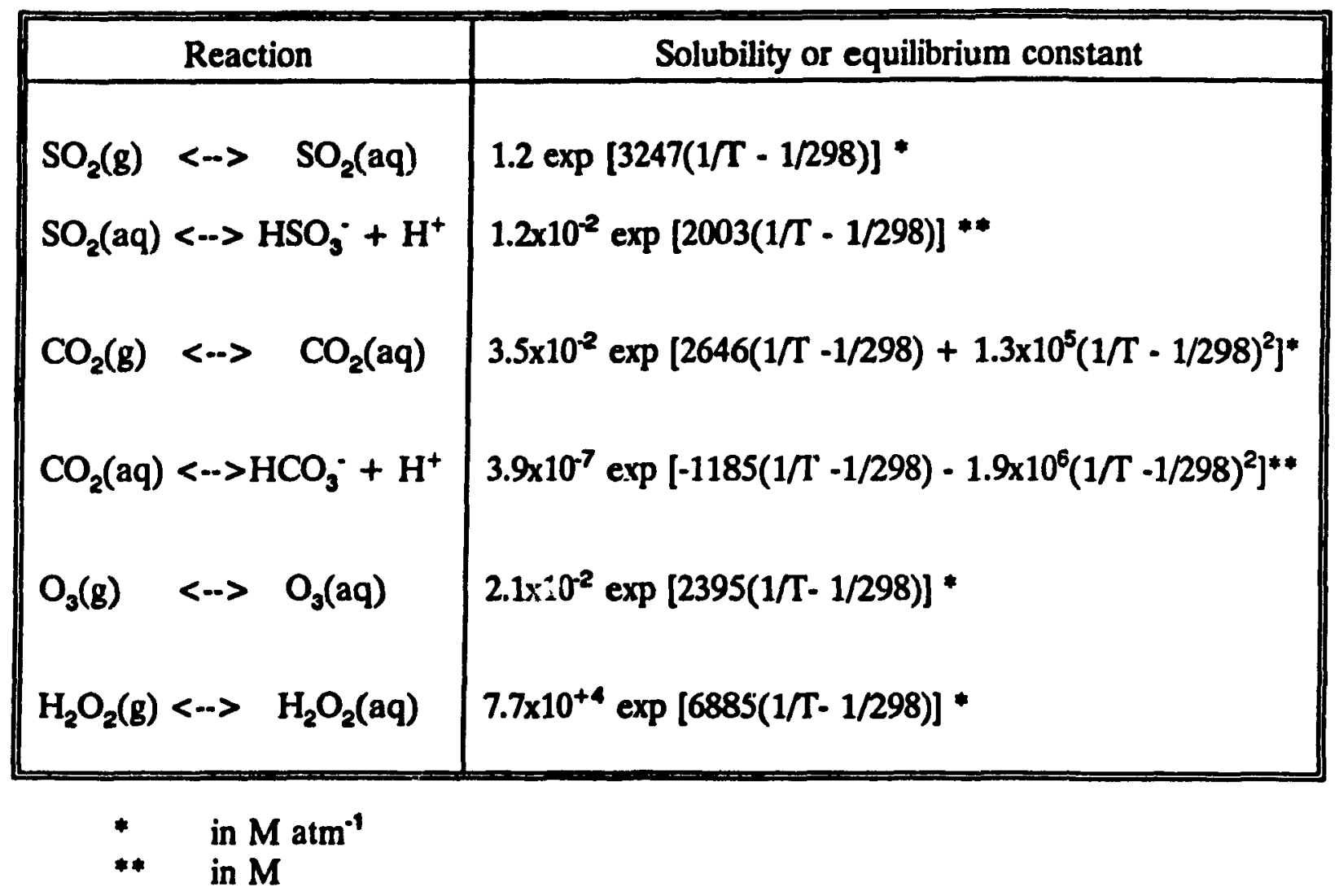

\subsubsection{Aerosol composition and scavenging}

Aerosol composition is different in the two models. In the cloud chemistry model only two types of aerosols are considered: ammonium sulphate $\left(\left(\mathrm{NH}_{4}\right)_{2} \mathrm{SO}_{4}\right)$ and sulphuric acid $\left(\mathrm{H}_{2} \mathrm{SO}_{4}\right)$. The relative proportion of this two kinds of aerosol is 
a parameter of the model. ADOM includes ammonium nitrate aerosol $\left(\mathrm{NH}_{4} \mathrm{NO}_{3}\right)$, which is always present in ADOM calculations. It includes also three types of sulphur aerosols, the two mentioned and also ammonium bisulphate $\left(\mathrm{NH}_{4} \mathrm{HSO}_{4}\right)$. Ammonium sulphate is only present for high concentrations of ammonia in the ambient air, and if ammonium sulphate is present the other two sulphate aerosols are assumed to be absent. At intermediate concentrations of ammonia only ammonium bisulphate is allowed to be present. At low concentrations of ammonia both sulphuric acid and ammonium bisulphate are allowed to be present in relative concentrations that depend on the ammonia concentration. In order to determine specifically which situation actually occurs in reality, not concentration but total ammonia in the domain is compared with total sulphate and nitrate in the domain.

In the cioud chemistry model, aerosol scavenging can occur throughout the cloud lifetime but not all of the aerosol is necessarily nucleated. In the ADOM scavenging module all aerosols present in the active part of the cloud nucleate and this takes place just after cloud formation. This feature indicates that the particular composition of aerosols in ADOM is unimportant. What really matters is the initial concentration of sulphates, ammonia and nitric acid. How these species are combined to form aerosols is not important, because all aerosol is immediately found in the cloud water as soon as cloud forms. 


\subsubsection{Aqueous-phase oxidation reactions}

Two pathways for oxidation: by ozone, and by hydrogen peroxide, are considered in the three dimensional cloud chemistry model. The same possibilities of oxidation exist in ADOM but two other methods are included as well: oxidation by the organic peroxide and by oxygen in the presence of catalysts. These two methods contribute insignificantly to total oxidation for most atmospheric conditions. Some preliminary tests have been done to investigate the relative importance of oxidation pathways in the ADOM scavenging module. If catalytic oxidation was excluded the concentrations of the species of interest remained almost unchanged, the changes being less then $0.1 \%$. As the initial concentration of organic peroxide is set equal to zero (according to previous considerations) this pathway of oxidation can not be important. So for the comparison purposes these two pathways were excluded from ADOM. The cloud chemistry model oxidation rates for ozone and hydrogen peroxide were updated to ADOM values. Finally in both models there are exactly the same expressions for oxidation rates by ozone and hydrogen peroxide as presented in table 2.4 (the two first expressions). 


\section{Chapter 3 \\ NUMERICAL EXPERIMENTS}

\subsection{Introduction}

In the same environment, characterised by its temperature, relative humidity and velocity profiles, two different convective clouds are simulated. They will be subsequently referred to as CLOUD A and CLOUD B. The environmental dynamical properties, as well as the characteristics of both clouds, are given in section 3.2.1 The Cloud Dynamics Simulation.

CLOUD A and CLOUD B, both simulated by the cloud dynamics model, are allowed to develop in twelve different chemical environments each. The chemical environment is characterised by the set of initial concentration profiles of chemical species. The choice of twelve ensembles of initial concentration profiles is presented in section 3.2.2 - The Cloud Chemistry Simulation.

Twenty four different cases, simulated with cloud chemistry model, are then simulated by the scavenging module of ADOM. The problem of generating equivalent 
initial conditions, necessary to run the model, is addressed in section 3.3 - The ADOM Scavenging Module Simulation, jointly with a brief presentation of the output.

\subsection{Three-dimensional Cloud Chemistry Model Simulation}

\subsubsection{Cloud Dyramics Simulation}

(a) Initial conditions

The initial temperature and humidity profiles, representing conditions similar to these studied by Tremblay (Tremblay, 1985), are plotted on the tephigram (figure 3.1) and the velocity profile on the hodograph (figure 3.2 ). The pressure at the surface equals $975 \mathrm{mb}$. The autoconversion threshold is fixed at $1 \mathrm{~g} / \mathrm{m}^{3}$. No rain diffusion is permitted in the model. The cloud formation is initiated by a saturated humidity impulse of radius $2 \mathrm{~km}$. Two different positions of the initial humidity impulse allow the simulation of two different convective clouds: CLOUD A originates from the impulse being located between 1350 and $2550 \mathrm{~m}$; CLOUD B is generated by the impulse situated between 1050 and $2250 \mathrm{~m}$. 


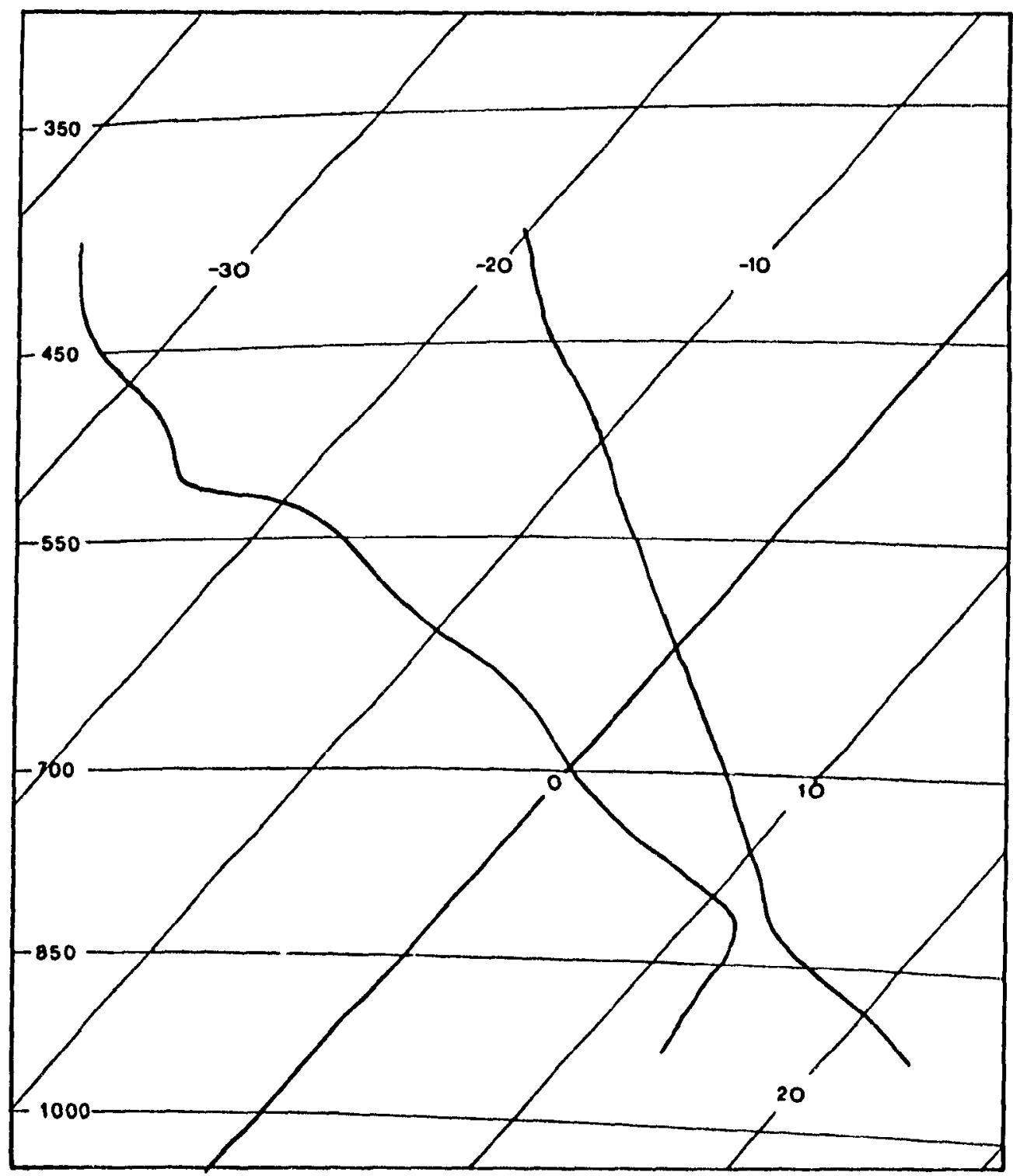

Figure 3.1 Temperature and humidity profiles plotted on the tephigram 


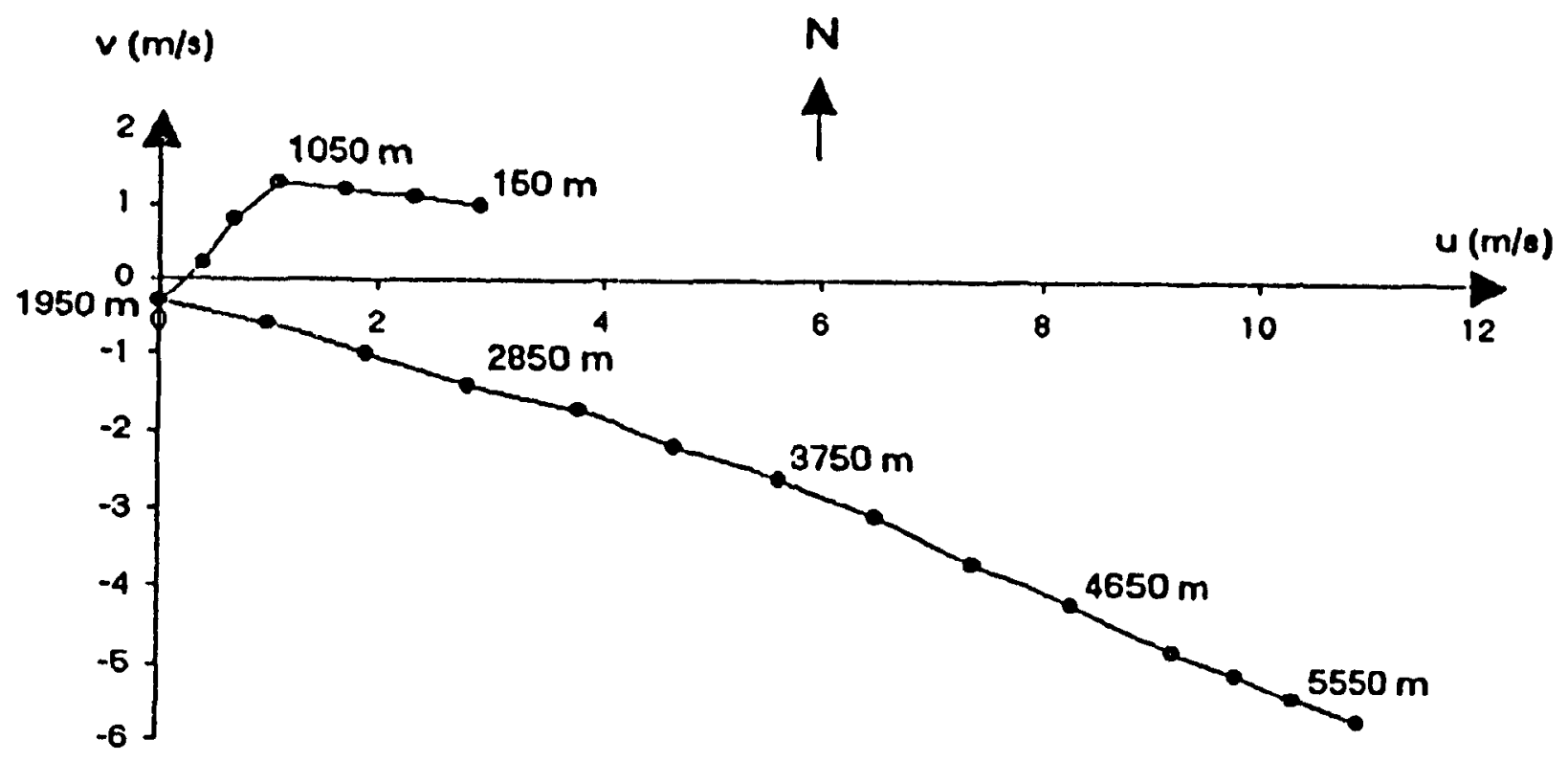

Figure 3.2 Hodograph of the initial horizontal wind

(b) Model results

Both clouds have their base at the same level, $1350 \mathrm{~m}$. The top of CLOUD $A$ is located at about $3450 \mathrm{~m}$ and of the CLOUD B at about $4350 \mathrm{~m}$. The estimated fractions of the horizontal area of the domain covered with CLOUD A and CLOUD B are both about $15 \%$. The time evolution of clouds, expressed by the cumulative cloud water content is presented in figures 3.3 and 3.4, the evolution of rain expressed by the cumulative rain water content is displayed in figures 3.5 and 3.6. The rainfall rate as a function of time, averaged over the cloudy area, for both clouds, is shown in figures 3.7 and 3.8. The properties of CLOUD A differ from those of CLOUD B only quantitatively, the character of the time dependence being almost the same (figures 3.3 to 3.8 ). 


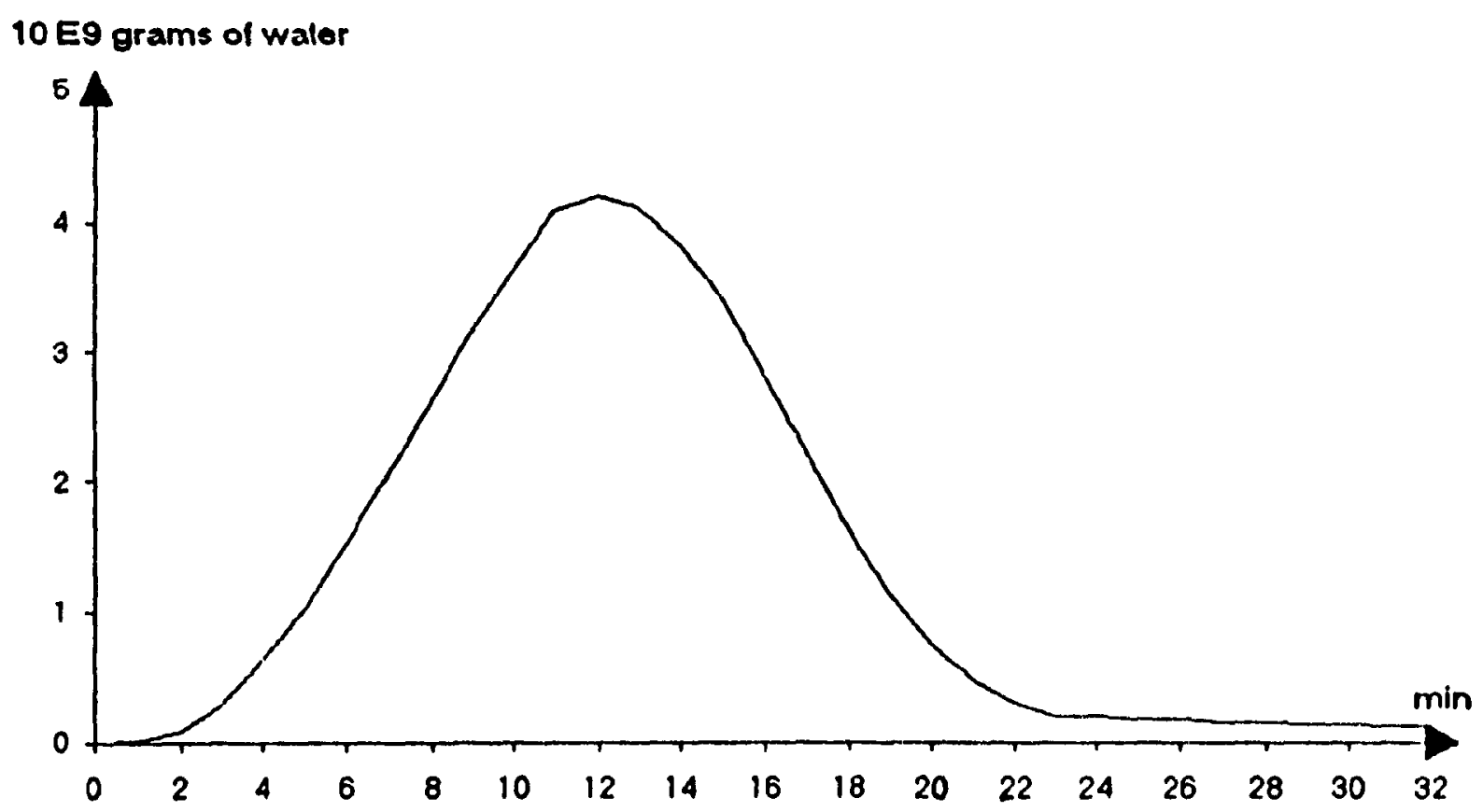

Figure 3.3 Variation of the total cloud water content of CLOUD A with time

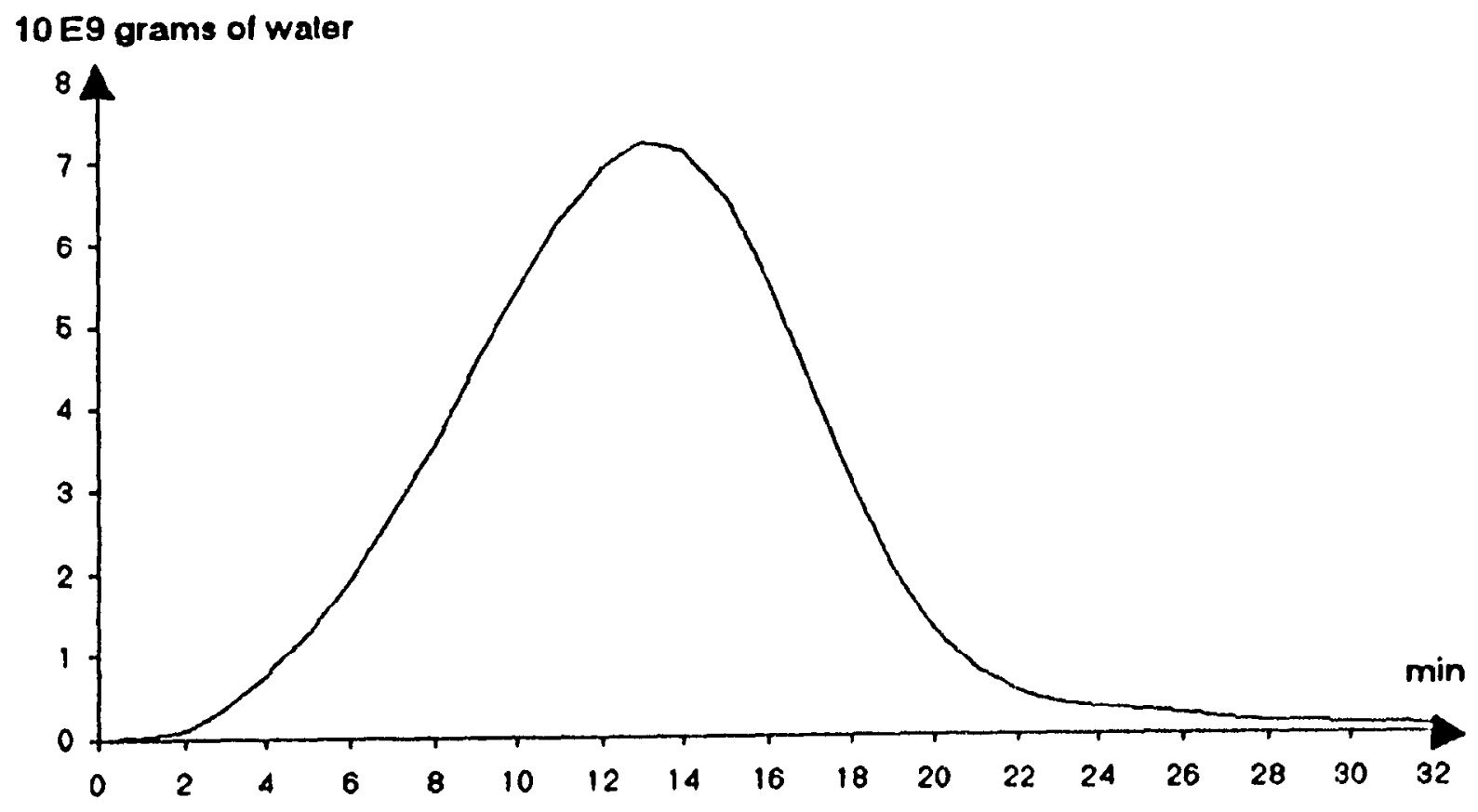

Figure 3.4 Variation of the total cloud water content of CLOUD B with time 


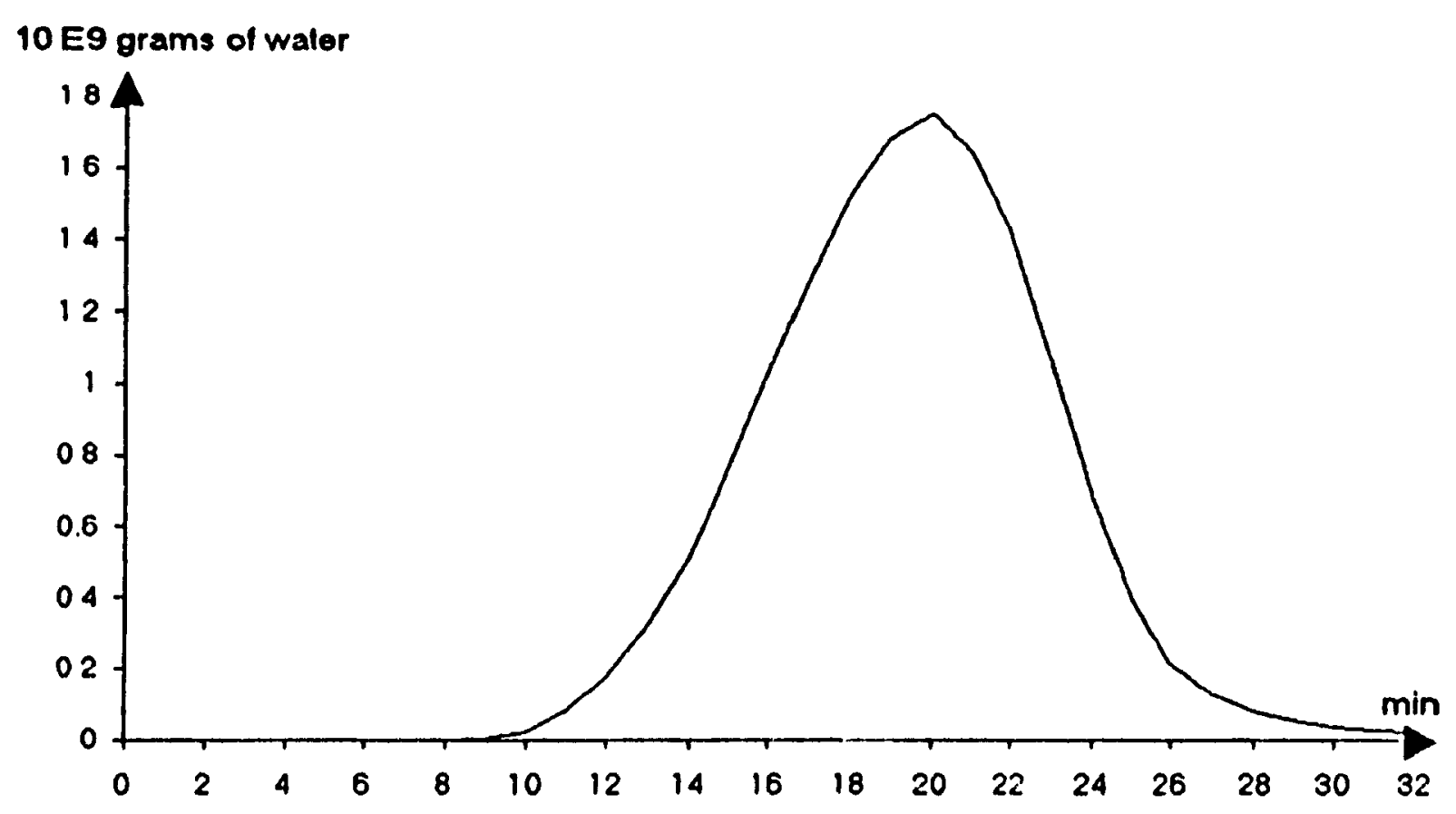

Figure 3.5 Variation of the total rain water content of CLOUD A with time

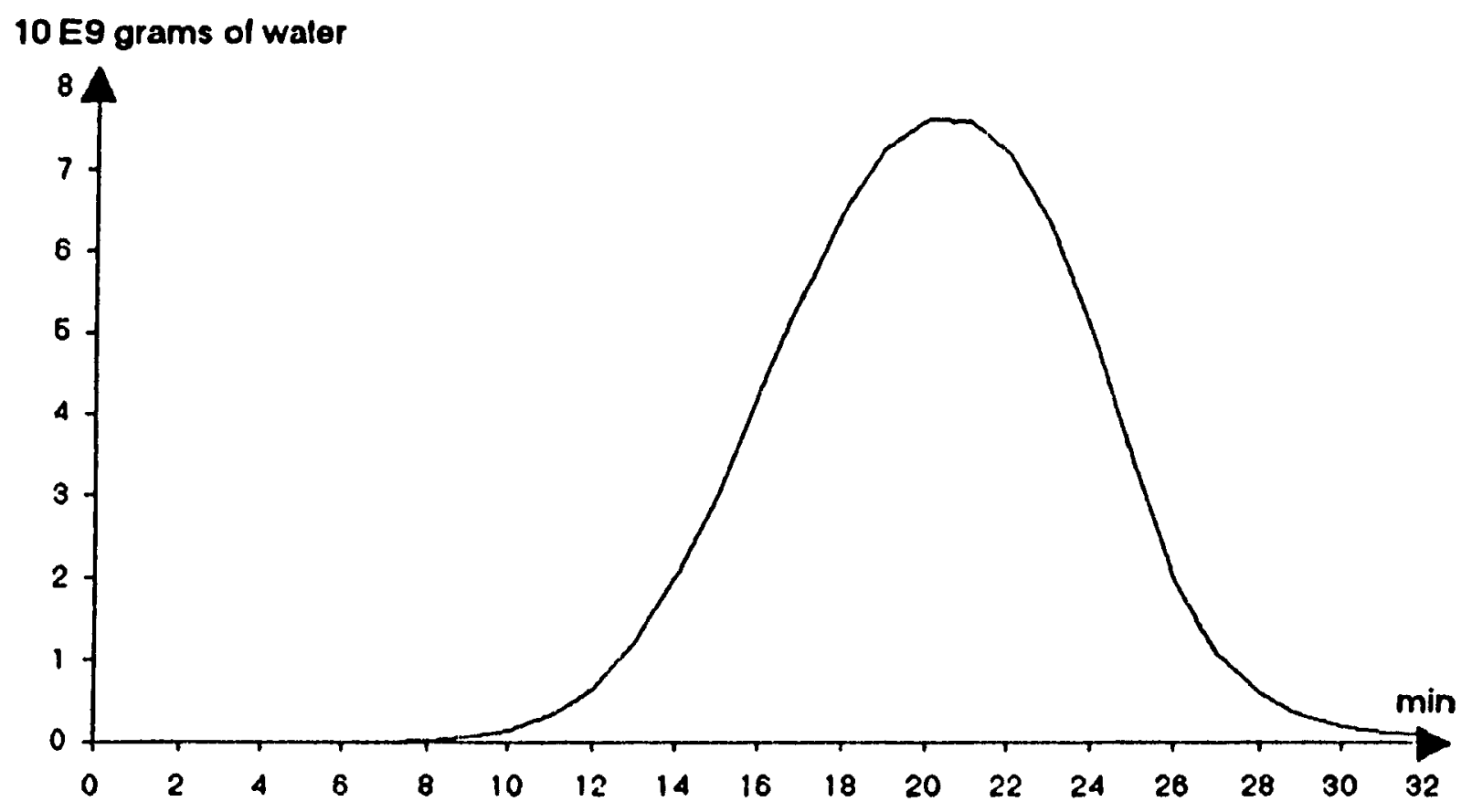

Figure 3.6 Variation of the total rain water content of CLOUD B with time 


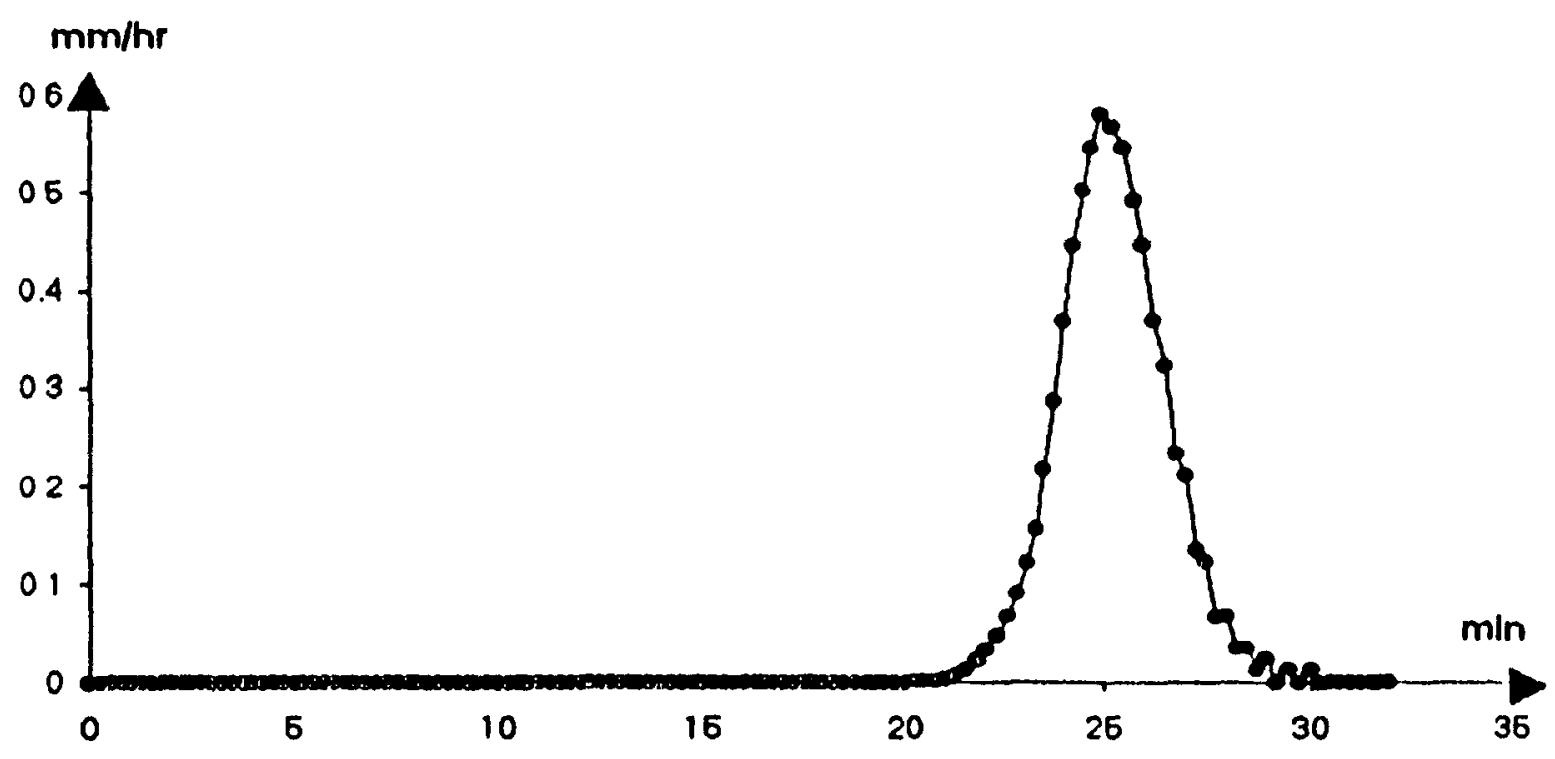

Figure 3.7 Variation of the total precipitation rate of CLOUD A with time

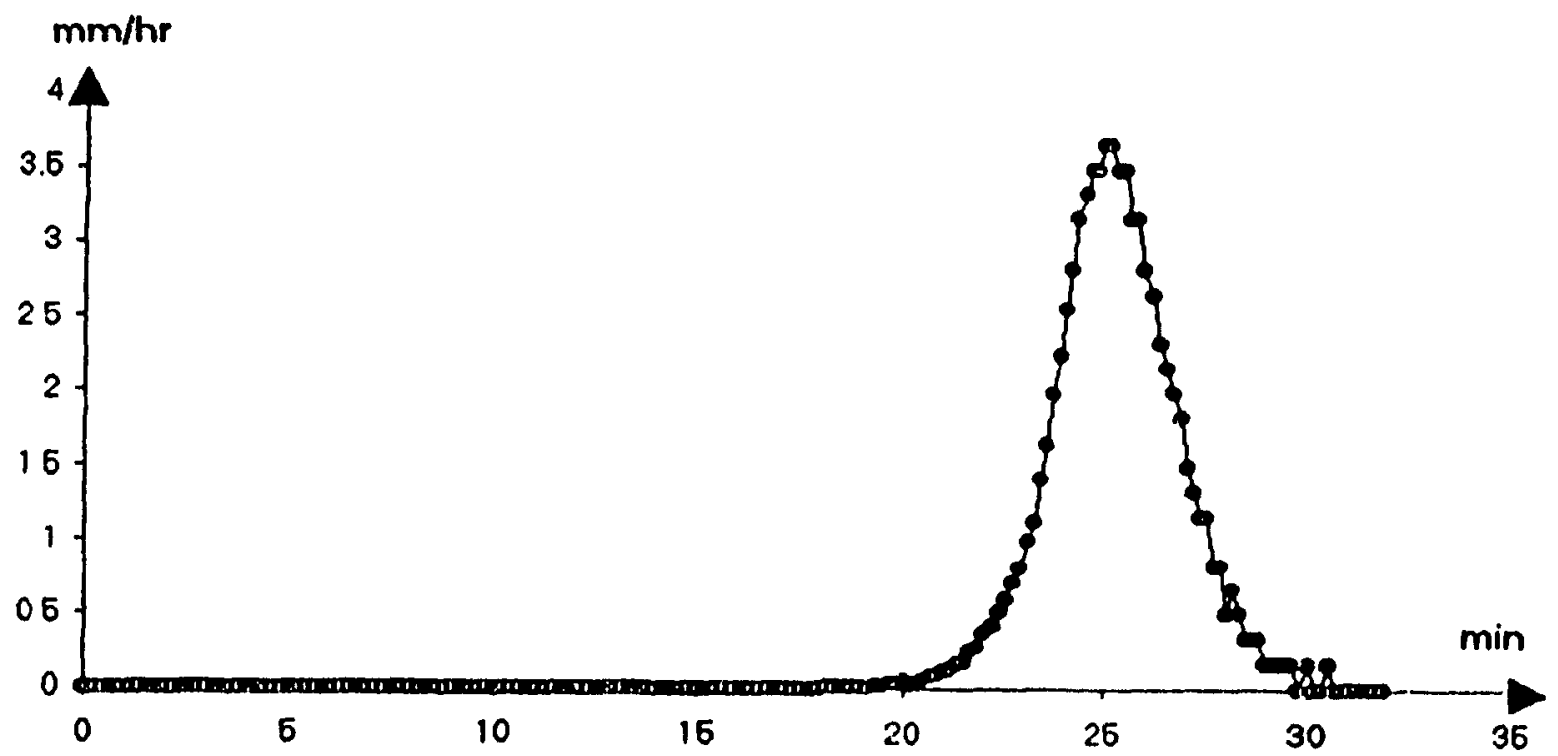

Figure 3.8 Variation of the total precipitation rate of CLOUD B with time 
Both clouds last about 30 minutes. The total amount of water deposited at the surface is $6.46 \times 10^{\circ}$ and $4.71 \times 10^{\circ}$ grams for CLOUD A and CLOUD B, respectively. This gives in average 0.0045 and $0.0327 \mathrm{~mm}$ of precipitation for the whole domain and 0.03 and $0.22 \mathrm{~mm}$ of precipitation for the cloud covered area. The peak value of precipitation rate is equal to 0.6 and $4.0 \mathrm{~mm} / \mathrm{hr}$ for CLOUD $A$ and CLOUD B, respectively.

The results of the cloud dynamics model provide the necessary input data to both the cloud chemistry model and the ADOM scavenging module. The cloud chemistry model requires the detailed description of the evolving cloud (at each time step), the ADOM scavenging module needs only some overall characteristics of the simulated cloud. Both models require also extra information about the initial profiles of chemical species.

\subsubsection{Cloud Chemistry Simulation}

(a) Initial conditions

The choice of the shape of concentration profiles and values of the concentrations at the surface are based upon field measurements (Seinfeld, 1986; Strapp et al., 1988; Kelly et al., 1989; Daum et al., 1989; Meagher et al., 1990; Isaac et al., 1990; Berkowitz, 1991; Dollard et al., 1991; Leaitch et al., 1991). The concentration of chemicals is assumed uniform in the mixing layer and then 
decreasing with height (with the exception of hydrogen peroxide and ozone). The particular values of concentrations at the surface were selected to be in agreement with typical values reported in the literature.

In all twelve cases considered in this study the concentration of nitric acid vapour is always the same and equals $1 \mathrm{ppbv}$ at the surface, then it decays aloft. The concentration of ammonia gas takes two values $1 \mathrm{ppbv}$ and $4 \mathrm{ppbv}$, again these are surface concentrations and a decrease aloft is assumed. Hydrogen peroxide concentration is kept constant through the vertical domain of the model, three different values being considered, $0.2,1.0$ and $4.0 \mathrm{ppbv}$. Three different values of sulphur dioxide concentration $(1.0,5.3,20.0 \mathrm{ppbv}$ at the surface) and three values of sulphate concentration $\left(1.0,5.2,20.0 \mu \mathrm{g} / \mathrm{m}^{3}\right.$ at the surface) are chosen.

The concentration of the species mentioned above (with the exception of hydrogen peroxide) is constant below cloud base and then is assumed to decay exponentially with height. The scale height is taken to be about one kilometre (Giles, 1987).

By combining selected values of species concentration, twelve different cases are created. They characterise low, moderately and highly polluted atmospheres. Case 1 describes the cleanest atmospheric conditions, followed by cases that can be obtained from the case 1 by changing the concentration of one or two species at a 
time. The following convention is adopted in subsequent descriptions:

D stands for sulphur dioxide

S stands for sulphate

P stands for hydrogen percxide

A stands for ammonia.

Case $1+D$ characterises the environment with initial concentration of all chemicals the same as for the case 1 except sulphur dioxide. The concentration of this species is increased and so on with other species. All six cases $(1,1+D, 1+S, 1+P, 1+A$ and $1+D+P)$ are described in table 3.1 . They characterise low to moderately polluted atmospheric conditions.

Table 3.1 Initial concentrations of chemicals, expressed in $\mu \mathrm{g} / \mathrm{m}^{3}$ for $\mathrm{SO}_{4}$ and in ppbv for all other species

\begin{tabular}{|c|c|c|c|c|c|c|}
\hline \multirow{2}{*}{ Chemical } & \multicolumn{7}{|c|}{ Case } \\
\cline { 2 - 7 } & 1 & $1+\mathrm{D}$ & $1+\mathrm{S}$ & $1+\mathrm{P}$ & $1+\mathrm{A}$ & $1+\mathrm{D}+\mathrm{P}$ \\
\hline $\mathrm{SO}_{2}(\mathrm{D})$ & 1.0 & 5.3 & 1.0 & 1.0 & 1.0 & 5.3 \\
\hline $\mathrm{SO}_{4}(\mathrm{~S})$ & 5.2 & 5.2 & 20.0 & 5.2 & 5.2 & 5.2 \\
\hline $\mathrm{H}_{2} \mathrm{O}_{2}(\mathrm{P})$ & 0.2 & 0.2 & 0.2 & 1.0 & 0.2 & 1.0 \\
\hline $\mathrm{HNO}_{3}$ & 1.0 & 1.0 & 1.0 & 1.0 & 1.0 & 1.0 \\
\hline $\mathrm{NH}_{3}(\mathrm{~A})$ & 1.0 & 1.0 & 1.0 & 1.0 & 4.0 & 1.0 \\
\hline
\end{tabular}


Table 3.2 Initial concentrations of chemicals, expressed in $\mu \mathrm{g} / \mathrm{m}^{3}$ for SO, and in ppbv for all other species

\begin{tabular}{||c|c|c|c|c|c|c||}
\hline \multirow{2}{*}{ Chemical } & \multicolumn{7}{|c|}{ Case } \\
\cline { 2 - 7 } & 2 & $2+\mathrm{D}$ & $2+\mathrm{P}$ & $2+\mathrm{D}+\mathrm{P}=3$ & $3-\mathrm{S}$ & $3+\mathrm{A}$ \\
\hline $\mathrm{SO}_{2}(\mathrm{D})$ & 5.3 & 20.0 & 5.3 & 20.0 & 20.0 & 20.0 \\
\hline $\mathrm{SO}_{4}(\mathrm{~S})$ & 20.0 & 20.0 & 20.0 & 20.0 & 5.2 & 20.0 \\
\hline $\mathrm{H}_{2} \mathrm{O}_{2}(\mathrm{P})$ & 1.0 & 1.0 & 4.0 & 4.0 & 4.0 & 4.0 \\
\hline $\mathrm{HNO}_{3}$ & 1.0 & 1.0 & 1.0 & 1.0 & 1.0 & 1.0 \\
\hline $\mathrm{NH}_{3}(\mathrm{~A})$ & 1.0 & 1.0 & 1.0 & 1.0 & 1.0 & 4.0 \\
\hline
\end{tabular}

The same convention is valid for table 3.2, where six cases are presented. They characterise polluted to highly polluted conditions. Case 2 describes the lowest level of pollution among the six cases from the table 3.2. Three cases start from number $2(2,2+D, 2+P)$, then case $2+D+P$ is renamed case 3 and subsequent cases are 3-S and 3+A.

(b) Model results

CLOUD A, then CLOUD B, are allowed to develop in twelve different chemical environments each (see tables 3.1 and 3.2). Twenty four sets of model results are obtained this way. These data provide the reference values for model comparisons. They will be presented jointly with the results of the ADOM scavenging module simulation. 


\subsection{ADOM Scavenging Module Simulation}

\subsubsection{Model initialization}

The scavenging module is only a part of $A D O M$. As a consequence the values of some of its initial parameters have to be fixed in order to assure the compatibility of ADOM modules. These parameters specify the temporal and spatial structure of the model. They are specified below:

- simulation and printing time steps ( $1 \mathrm{hr}$ and $5 \mathrm{~min}$, respectively), the value of the printing time step can be changed if necessary;

- number of vertical layers (12);

- distances between vertical layers (see table 2.5);

- horizontal grid ( $33 \times 33$ cells, see figure 2.2 ).

The remaining initial parameters are specific for the ADOM scavenging module. They can be associated in three groups. The parameters from a particular group describe:

(1) the dynamical and chemical properties of the environment;

(2) the cloud properties that are required by the cloud physics submodule;

(3) the details of the aqueous-phase chemistry mechanism.

(1) The dynamical and chemical properties of the environment are characterised 
by the initial values of temperature, pressure, and humidity for the 12 vertical levels (coinciding with the centres of vertical layers of $A D O M$ ), and initial concentrations of modelled chemicals, specified for the same 12 vertical levels. Those data can be determined as soon as the initial data for the 23 vertical levels of the cloud chemistry model have been specified. As the cloud chemistry model vertical resolution is not the same as ADOM, a spline interpolation procedure has been applied to compute initial profiles for the $12 \mathrm{ADOM}$ levels. At this point it is worth mentioning that the determination of the equivalent initial concentrations profiles of sulphate and ammonia in ADOM has to be done carefully. Sulphate in the air in the cloud chemistry model is assumed to be composed of sulphuric acid and ammonium sulphate. Consequently, for a particular value of the initial concentration of sulphate we have both an amount of $\mathrm{SO}_{4}{ }^{-}$and an amount of $\mathrm{NH}_{4}{ }^{+}$that are available for scavenging. Sulphate in $\mathrm{ADOM}$ means only $\mathrm{SO}_{4}{ }^{-}$ions. Therefore, when the initial concentrations of species for ADOM are specified, the concentration of ammonia gas must be increased to account for ammonium sulphate in the cloud chemistry model.

(2) The cloud physics submodule requires the values of the large and small scale precipitation rates and also the values of cloud parameters: cloud cover, cloud base and optionally cloud top location, and finally the active cloud fraction.

The large scale precipitation rate is taken to be zero. This assumption guarantees that the observed precipitation is due to the convective activity rather then stratiform cloud. 
The small scale precipitation rate as well as the cloud characteristics can be specified only after the cloud dynamics model run. The small scale precipitation rate is understood in the model as a mean value for both the ADOM grid square and the simulation time step. As discussed in section 2.4 .2 , the values of the small scale precipitation rate and cloud cover, determined by the cloud dynamics model, can be used without any changes to initialize ADOM. The values of the small scale precipitation rates are equal $0.0045 \mathrm{~mm} / \mathrm{hr}$ for CLOUD A and $0.0327 \mathrm{~mm} / \mathrm{hr}$ for CLOUD B. The fraction of the domain covered by clouds is $15 \%$. The cloud base is located at $1350 \mathrm{~m}$, for both clouds, CLOUD A top is at $3450 \mathrm{~m}$, CLOUD B top is at $4350 \mathrm{~m}$.

The last initial parameter to be specified, is the active cloud fraction. This is an internal parameter for the Raymond and Blyth mixing model. ADOM results are insensitive to this parameter for the values of precipitation rates and cloud cover considered. This parameter may also be determined by the model itself.

(3) The details of the aqueous-phase chemistry calculations may be modified setting the values of certain switches:

(a) chemistry calculations;

(b) ice phase calculations;

(c) hydrogen peroxide oxidation;

(d) oxygen oxidation catalyzed by iron and manganese;

(e) organic peroxide oxidation. 
The choice of flags is made according to the general properties of the cloud chemistry model.

(a) This switch determines whether cloud chemistry calculations are turned on, or if only mixing is allowed without any chemical reactions taking place. Chemical reactions are turned on.

(b) This switch determines whether ice phase calculations are included. In our calculations ice phase calculations are excluded. CLOUD $\mathrm{A}$ is a warm cloud and the environmental temperature at the top of CLOUD B is about $-6^{\circ} \mathrm{C}$. The ice phase, even if present for CLOUD B, can not significantly influence model results, this phase can occupy only a small fraction of the cloud volume. Sensitivity studies realised with the ADOM scavenging module to investigate the influence of the presence of the ice phase lead to the following conclusions. The wet-deposited amount of nitrates and ammonium ions is almost not influenced by the presence of the ice phase. The wet-deposited amount of hydrogen and sulphate ions, and also sulphur dioxide is decreased when the ice phase is present. This decrease does not exceed 10 percent, even for the cloud extending up to the $10 \mathrm{~km}$. Hydrogen peroxide demonstrates a strange behaviour. In the simulations that do not include the ice phase it is highly depleted within the cloud, and consequently only a very small amount of the hydrogen peroxide can be wet-deposited. This feature changes substantially if the ice phase calculations are turned on. Then, an important fraction of the cloud-dissolved hydrogen peroxide is trapped in ice crystals, and therefore it can not be depleted by oxidation processes. At the end of the simulation time step, 
this fraction of hydrogen peroxide is wet-deposited. Two simulations, with and without the ice phase in the model, give results that differ by few orders of magnitude as far as wet deposition of hydrogen peroxide is considered.

(c) Oxidation by hydrogen peroxide is included.

(d) and (e) Oxidation by oxygen (catalyzed by iron and manganese) and by organic peroxides are not included (see section 2.4.7).

\subsubsection{ADOM clouds}

The cloud base and top locations determined by the cloud dynamics model do not coincide with ADOM cell faces (table 2.5). Within ADOM the only allowed location of cloud base or top is the height of cell faces, therefore these initial parameters must be recomputed. Cloud base determined by the ADOM cloud physics submodule is located at $1497.2 \mathrm{~m}$. The top of CLOUD $\mathrm{A}$ is at $4741.6 \mathrm{~m}$ (ADOM II) and the top of CLOUD B is at $6894.5 \mathrm{~m}$ (ADOM III). CLOUD A, $2100 \mathrm{~m}$ thick, is represented now by a cloud $3244.4 \mathrm{~m}$ thick. CLOUD B, $3000 \mathrm{~m}$ thick, is represented by a cloud $5397.3 \mathrm{~m}$ thick. All these data are in table 3.3 .

A natural question arises: as the cloud top location determined by ADOM is a step function of the input value of the cloud lop location at which height does the jump occur? Is it close to values 3450 or $4350 \mathrm{~m}$ ? As it depends on the totality of initial conditions the answer can not be given on a theoretical basis, but has to be 
found by subsequent trials. The answer is as follows: lowering the input CLOUD A top location by $750 \mathrm{~m}$ and input CLOUD B top location by $500 \mathrm{~m}$ decreases in each case the cloud top height of the ADOM cloud by one layer. CLOUD B and another cloud B1 with a cloud top $500 \mathrm{~m}$ lower are represented by totally different ADOM clouds that differ in thickness by as much as $2153 \mathrm{~m}$ ! This shows how sensitive the ADOM clouds can be to the input cloud top height.

Table 3.3 Characteristics of simulated clouds

\begin{tabular}{|c|c|c|c|c|c|}
\hline Cloud & $\begin{array}{c}\text { Bottom } \\
(\mathrm{m})\end{array}$ & $\begin{array}{c}\text { Top } \\
(\mathrm{m})\end{array}$ & $\begin{array}{c}\text { Thickness } \\
(\mathrm{m})\end{array}$ & $\begin{array}{c}\text { LWC } \\
\left(\mathrm{g} / \mathrm{m}^{3}\right)\end{array}$ & $\begin{array}{c}\text { Total } \\
\text { water }(\mathrm{g})\end{array}$ \\
\hline \hline CLOUD A & 1350 & 3450 & 2100 & $0.85 *$ & $4.2 \times 10^{\circ} * *$ \\
CLOUD B & 1350 & 4350 & 3000 & $1.1 *$ & $7.4 \times 10^{\circ} * *$ \\
\hline ADOM I & 1497.2 & 3249.2 & 1752.0 & 0.186 & $3 \times 10^{\circ}$ \\
ADOM II & 1497.2 & 4741.6 & 3244.4 & 0.716 & $18 \times 10^{\circ}$ \\
ADOM III & 1497.2 & 6894.5 & 5397.3 & 1.40 & $46 \times 10^{\circ}$ \\
\hline
\end{tabular}

* max value of liquid water content during cloud development, mean value for level $300 \mathrm{~m}$ thick

** max value of condensed water during cloud development

In order to investigate the sensitivity of the model output to changes in cloud top location, the following computations are performed. CLOUD A, simulated by the cloud dynamics model, is represented by two ADOM clouds that differ only, in terms 
of input data, by the cloud top location. One of the clouds is obtained by assuming CLOUD A top location, the other by lowering (by $750 \mathrm{~m}$ ) the value of input cloud top location. The shallower cloud will be subsequently referred to as ADOM I, the deeper ADOM II.

Similarly CLOUD B is represented by two clouds: ADOM II, obtained this time by lowering the input cloud top location by $500 \mathrm{~m}$, and ADOM III obtained assuming CLOUD B top location. All five clouds are represented in the figure 3.9 and their important characteristics in table 3.3. It is obvious that the ADOM scavenging module simulates only one cloud if all its initial parameters are specified. Initial data obtained by the cloud dynamics model simulation correspond to the cloud ADOM II (for CLOUD A) and ADOM III (for CLOUD B). The two remaining clouds are introduced only for investigatory purposes. 


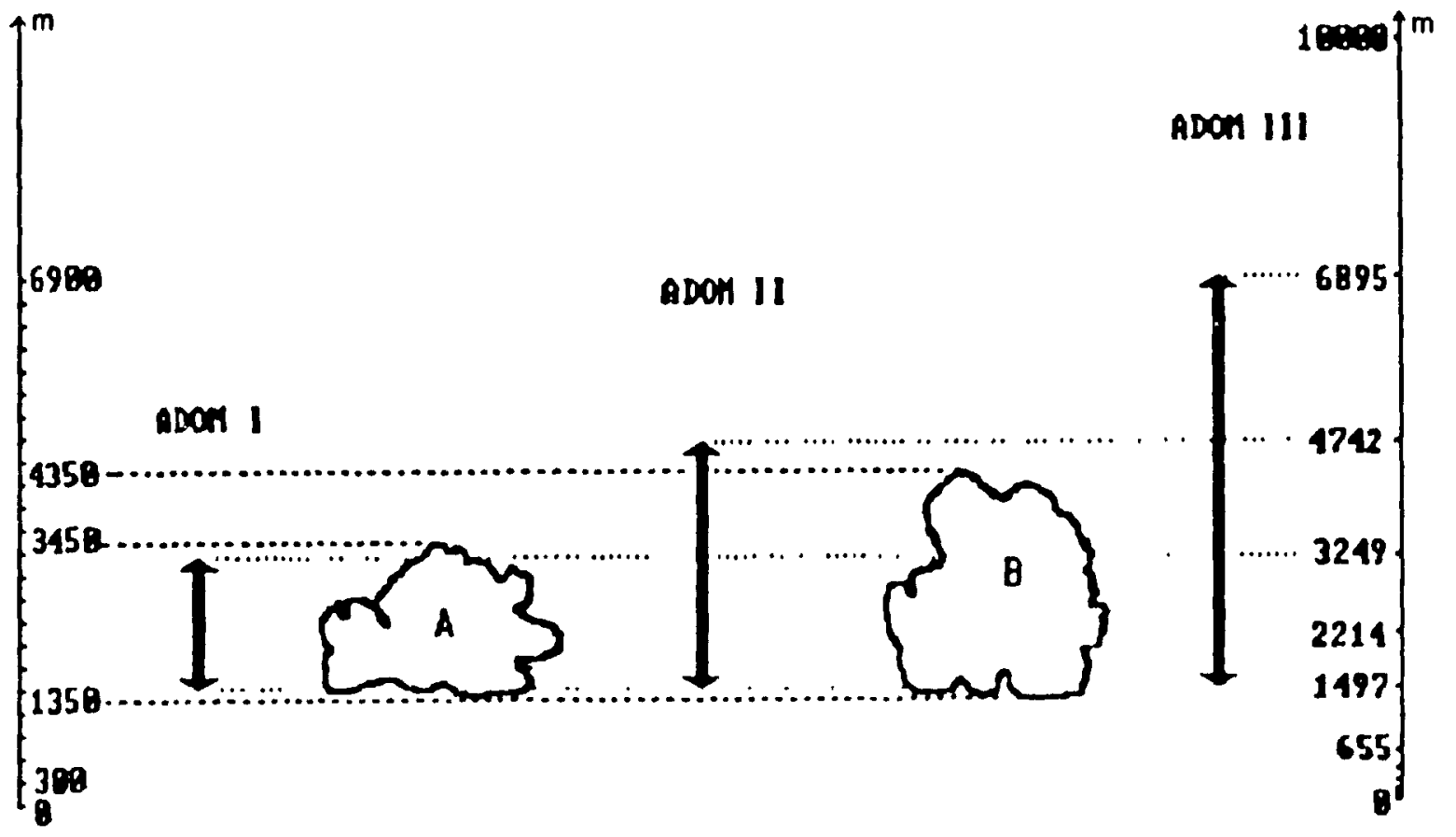

Figure 3.9 Vertical dimensions of the simulated clouds 


\subsubsection{Model output}

The following results of the ADOM scavenging module will be considered:

- fluxes of wet-deposited species (cumulative for the simulation time step);

- mean pH of rain;

- fraction of sulphate from in-cloud oxidation;

- total in-cloud oxidation (cumulative amount of sulph łte frcm oxidation);

- final concentration of chemical species in air at 12 model levels.

Exactly the same information can be obtained from results of cloud chemistry model simulation. The ADOM scavenging module is evaluated by comparing the different elements of the model output presented above with the corresponding values from the cloud chemistry model for the different chemical environments presented in section 3.2.2 (a).

The results of the cloud chemistry model simulations are reference values for comparison purposes. Their role is the same as field data in a standard model evaluation procedure. The closer are the numbers characterising the same quantity the better we assume is $A D O M$ validity. This validity is relative to the cloud chemistry model and this meaning will be implied throughout the remaining part of this study. 


\section{Chapter 4}

\section{EVALUATION OF THE ADOM SCAVENGING MODULE}

\subsection{Introduction}

In this study, the evaluation of the ADOM scavenging module is realized by inter-model comparisons. The results of the cloud chemistry model simulation are the reference values. As there is no general consensus about what can be judged an adequate approximation and acceptable discrepancy between model and reference values, the evaluation can not be free from subjectivity.

The wet-deposition of sulphate, nitrate and ammonium ions simulated by both models is presented in section 4.2. The rain acidity predictions are compared in section 4.3. Hydrogen peroxide wet deposition is described in section 4.4.

Total oxidation of S(IV) is given by both models, even though in the ADOM scavenging module in-rain oxidation is not taken explicitly into consideration. Nevertheless, the comparisons of total oxidation are useful. Both models provide also the necessary information for judging the relative importance of oxidation and 
nucleation, which is discussed in section 4.5.

In order to investigate further the ADOM performance, the values of the nondimensional quantity, fractional acidity (Schwartz, 1984), are analyzed in section 4.6.

Comments about the concentration of chemicals in the air simulated by both models are presented in section 4.7. A brief comparison of the output of ADOM for different clouds concludes this chapter.

There are three curves in the majority of figures presented in this chapter. One, denoted CLOUD A or CLOUD B, describes the cloud chemistry model results, the two others describe the ADOM scavenging module results. CLOUD A with ADOM II and CLOUD B with ADOM III constitute two pairs of clouds simulated by the two models for equivalent initial conditions, and consequently will be compared first. The height of the top of CLOUD A is however closer to the height of the top of ADOM I than ADOM II. The height of the top of CLOUD B is closer to the height of the top of ADOM II than ADOM III. This is why CLOUD A will be subsequently compared with ADOM I and CLOUD B with ADOM II. 


\subsection{Wet deposition of sulphate, nitrate and ammonium ions}

The amount of sulphate, nitrate and ammonium ions wet-deposited over the whole cloud chemistry model domain is presented in figures 4.1 to 4.8 . The cases (on $\mathrm{x}$ axis) are ordered according to the increasing values of sulphate wet deposition from the cloud chemistry model simulations (or according to the increasing values of discrepancies for sulphate wet deposition for figures 4.3 and 4.4).

\section{Sulphate}

The wet deposition of sulphate simulated by the cloud chemistry model can be compared with the ADOM scavenging module predictions in figure 4.1 (CLOUD $A$ and $A D O M$ II) and in figure 4.2 (CLOUD B and ADOM III), but the discrepancies existing between model predictions are better visualized in figures 4.3 and 4.4. In these figures the discrepancies are related to the cloud chemistry model predictions. For both clouds ADOM underpredicts sulphate deposition by up to $40 \%$ with typical discrepancies being of the order of $20 \%$. The biggest underprediction of wet-deposited sulphate occurs for the weakly polluted atmosphere with the enhanced concentrations of ammonia (case $1+\mathbf{A}$ ). The only case of ADOM overprediction occurs for the bigger cloud (CLOUD B) developing in the environment with an enhanced concentration of sulphur dioxide (case 1+D).

The wet-deposited amount of sulphate although systematically underpredicted in the ADOM scavenging module may be considered to be reasonably well modelled. 


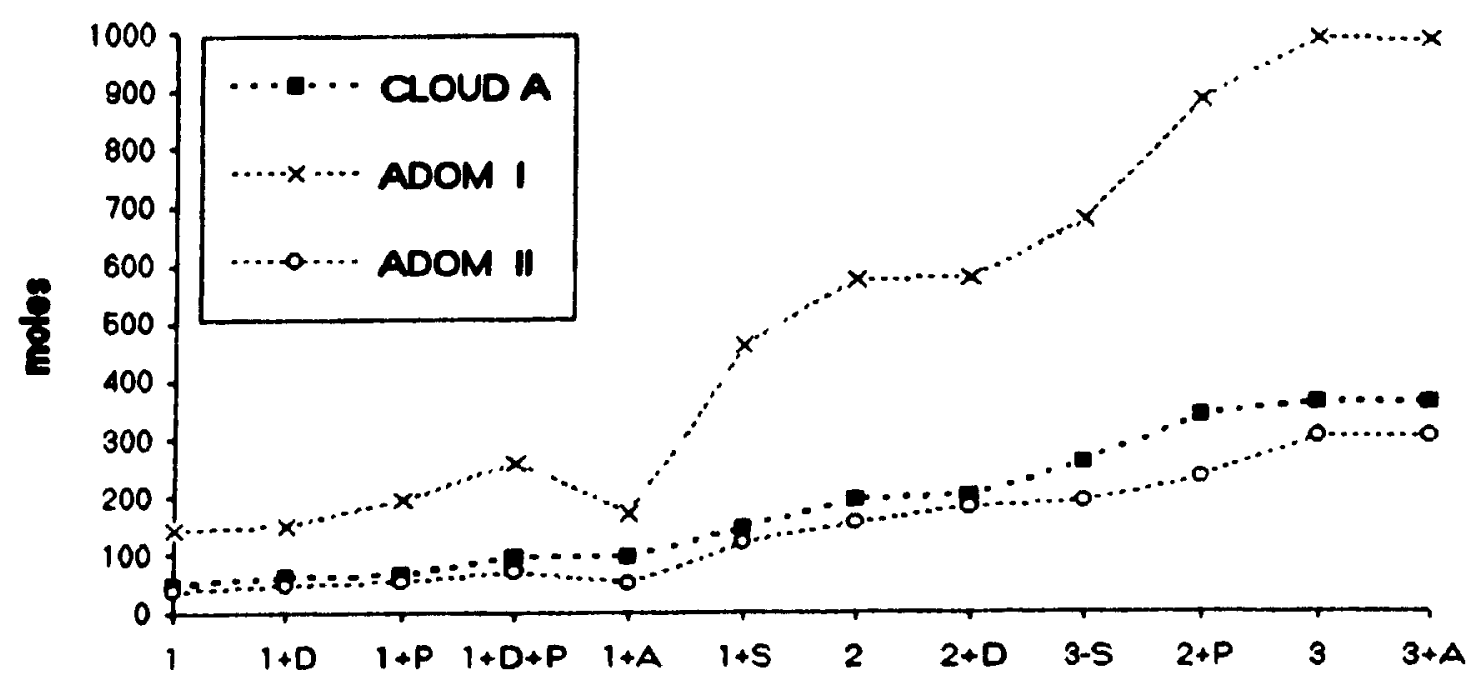

Figure 4.1 Wet deposition of sulphate by CLOUD A and corresponding ADOM clouds for different chemical environments

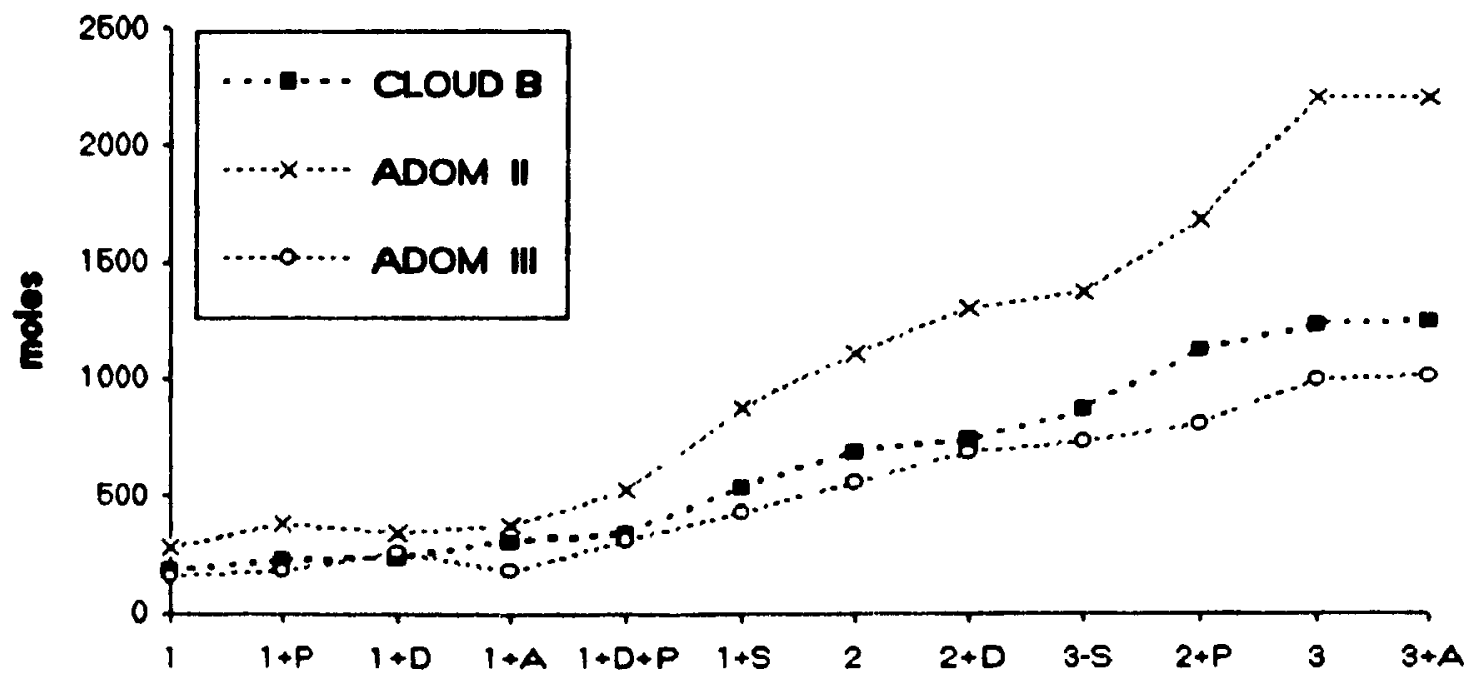

Figure 4.2 Wet deposition of sulphate by CLOUD B and corresponding ADOM clouds for different chemical environments 

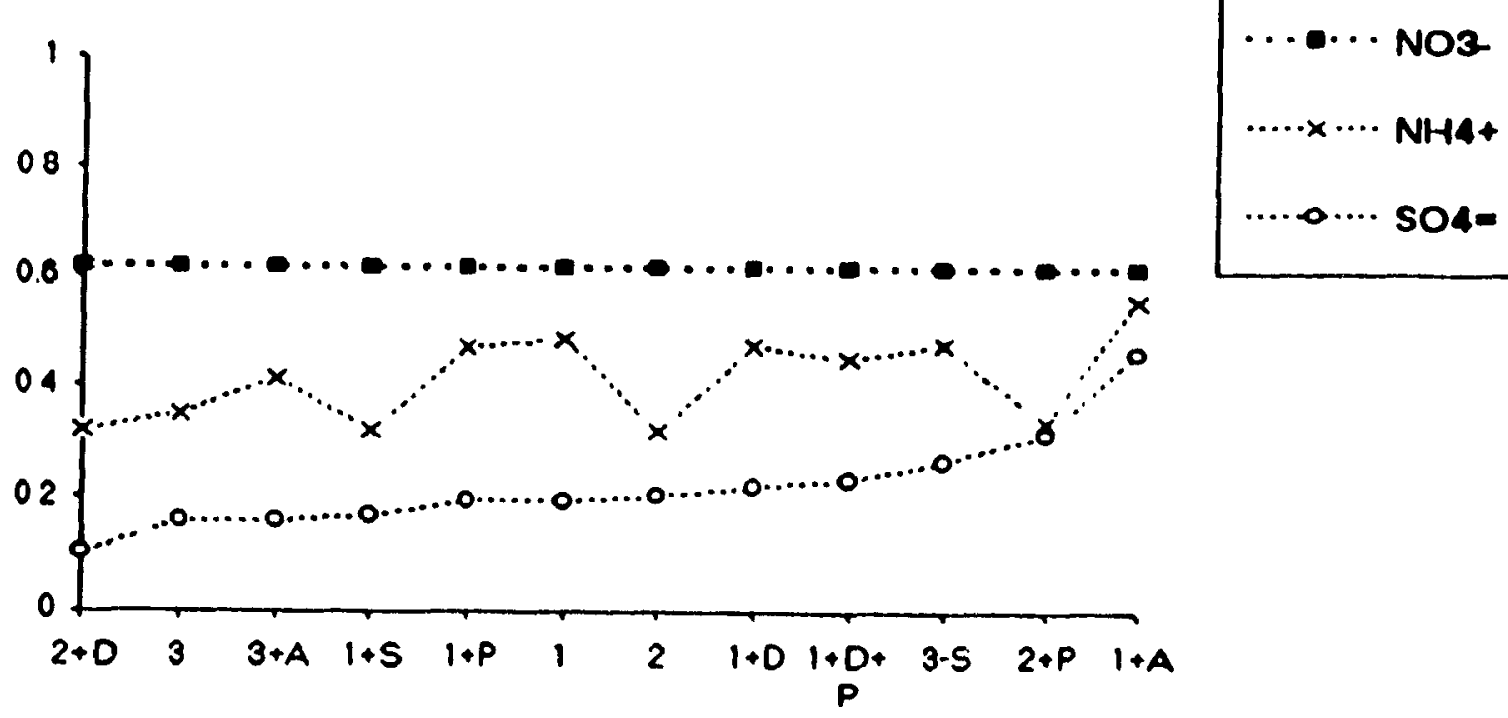

Figure 4.3 Wet deposition of sulphate, nitrate and ammonium, expressed as a difference between wet-deposited amount predicted by the cloud chemistry model for CLOUD $A$ and the corresponding ADOM cloud, divided by the cloud chemistry model prediction
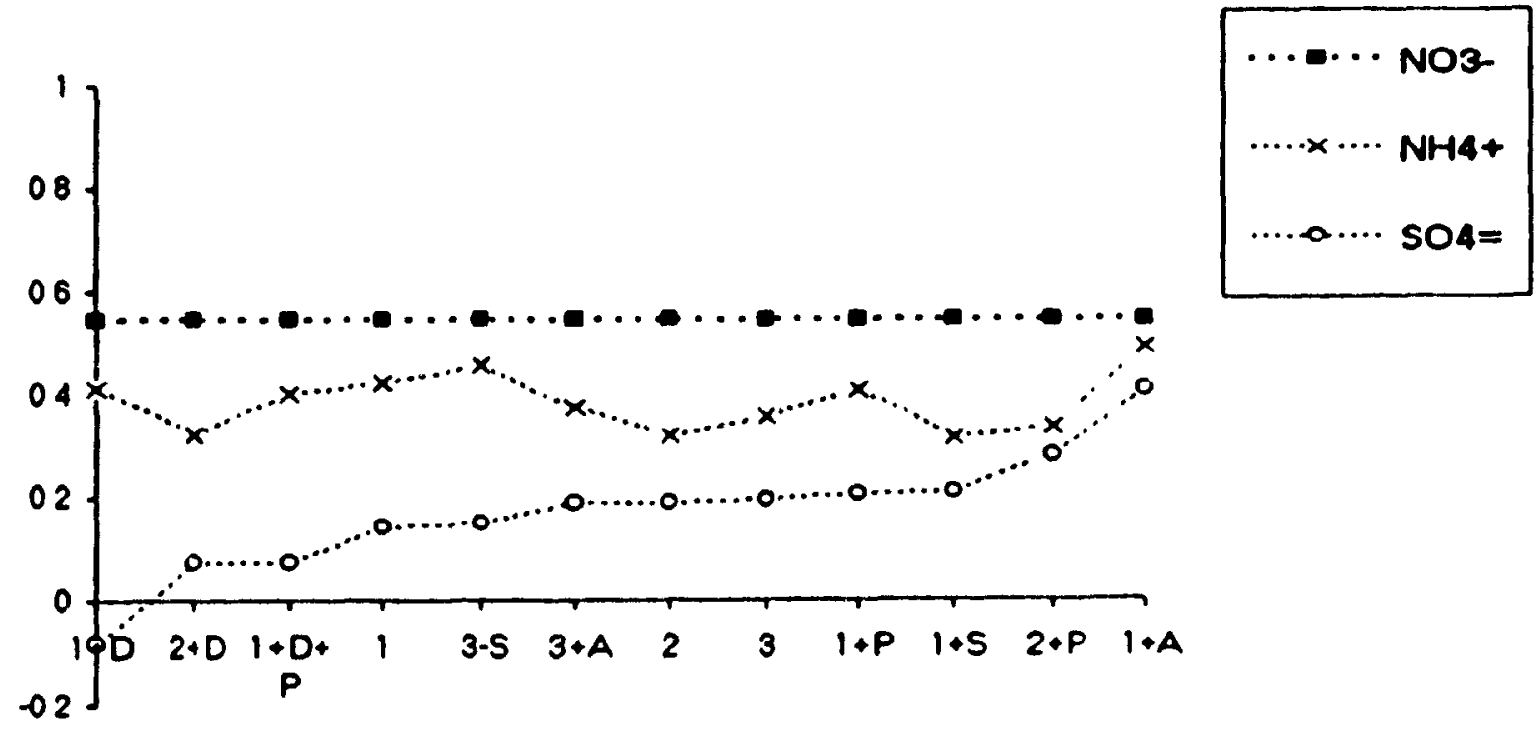

Figure 4.4 Wet deposition of sulphate, nitrate and ammonium, expressed as a difference between wet-deposited amount predicted by the cloud chemistry model for CLOUD B and the corresponding ADOM cloud, divided by the cloud chemistry model prediction 
The shallower ADOM clouds, ADOM I for CLOUD A and ADOM II for CLOUD B, significantly overpredict the amount of wet deposition of sulphate. This means that the concentration of sulphate in cloud water is bigger for the shallower ADOM cloud since the precipitation rate is the same for both shallower and deeper ADOM clouds that correspond to the same dynamic simulation.

The overprediction of the wet-deposited sulphate by the shallower cloud is generally greater then the underprediction by the deeper cloud.

\section{Nitrate}

The same conclusion is not valid for the wet deposition of nitrate (figures 4.5 and 4.6) where the shallower ADOM cloud always performs better, particularly for CLOUD B. For the deeper clouds ADOM underpredicts nitrate deposition by $60 \%$ (figures 4.3 and 4.4).

A few extra runs were performed to investigate the response of both models to doubling initial concentration of nitric acid in different environments. The results are not given in the figures. A significant change occurs in the an.ount of nitrate wetdeposited, which is twice as big as before. Ammonium ion wet deposition remains almost unchanged, sulphate and hydrogen ion wet deposition is slightly modified. These results confirm the passive role of nitric acid. As no source or sink of nitrate exists within both models its wet deposition depends on the initial concentration of 


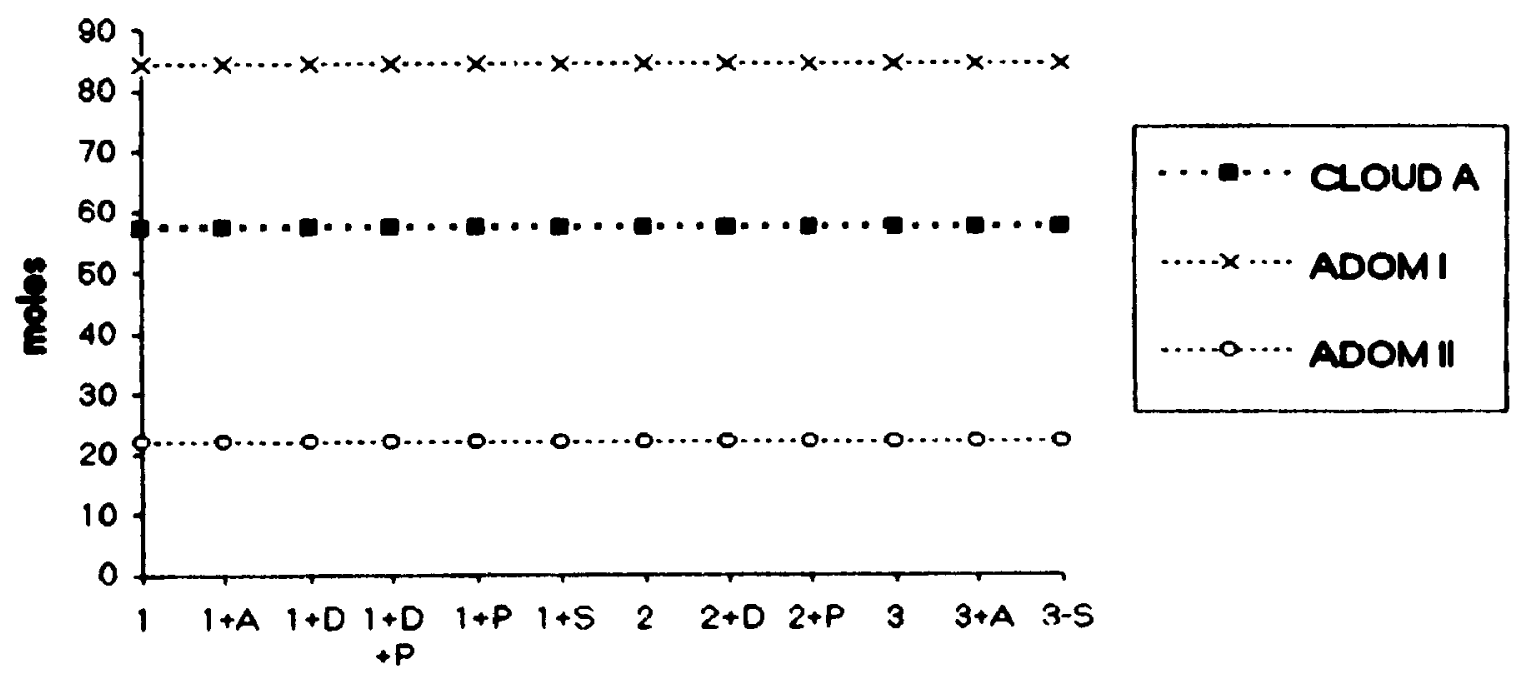

Figure 4.5 Wet deposition of nitrate by CLOUD $\mathrm{A}$ and corresponding ADOM clouds

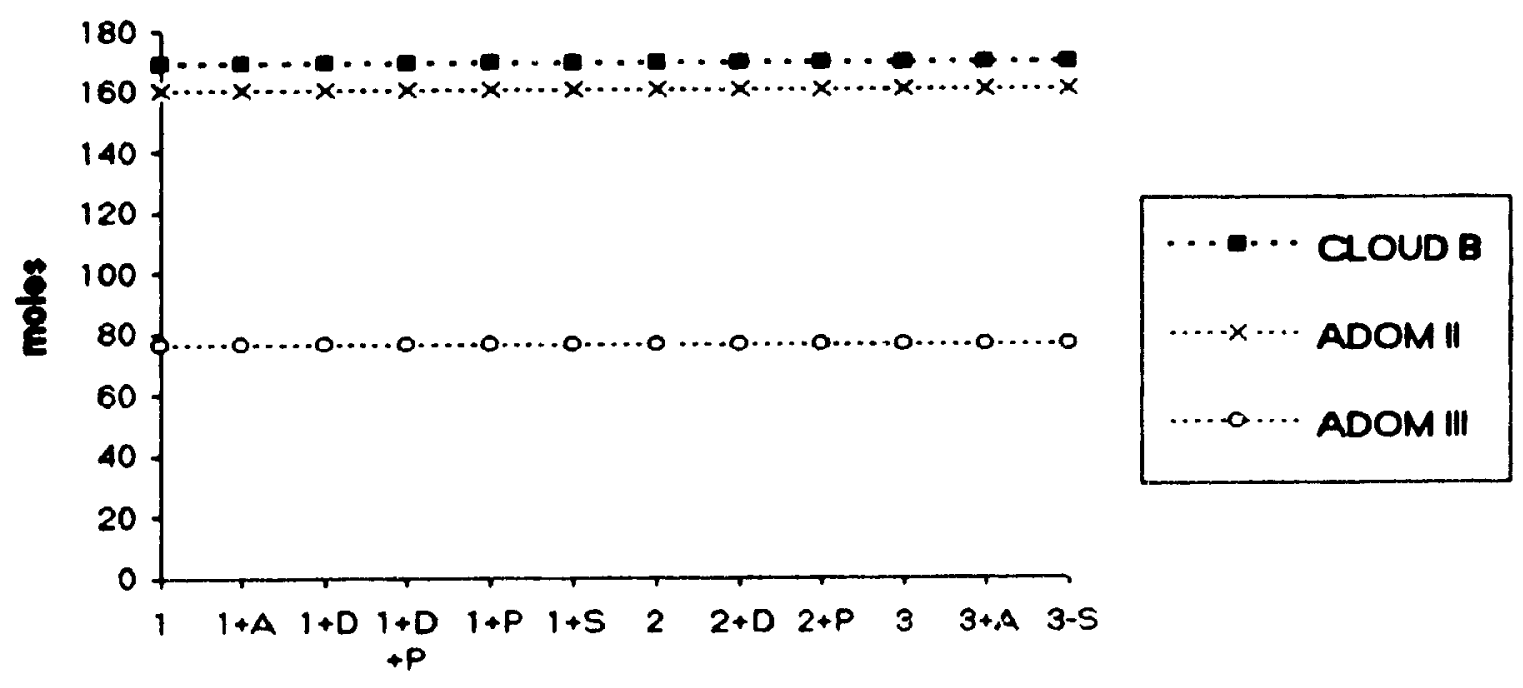

Figure 4.6 Wet deposition of nitrate by CLOUD E and corresponding ADOM clouds 
nitric acid and is almost independent of the concentrations of other chemicals. Thus it is expected that significant underprediction of nitrate wet deposition by the deeper ADOM aloud, and better agreement for the shallower cloud will be found regardless of the initial nitric acid concentration.

\section{A m monium}

The wet deposition of ammonium is presented in figures 4.7 and 4.8. The deeper ADOM cloud generally performs better. It underpredicts the wet-deposited amount of ammonium whereas the shallower ADOM cloud always overpredicts these quantities. For CLOUD B, however, and for the less polluted environments the shallower ADOM cloud represents the wet deposition of ammonium better than the deeper cloud.

The underprediction of ammonium deposited by the deeper ADOM cloud is at least $30 \%$ (figures 4.3 and 4.4 ). The biggest discrepancies between model results occur for the weakly polluted atmosphere with enhanced concentration of ammonia (case 1+A). The wet deposition of ammonium is described better than nitrate but not as well as sulphate wet deposition (figures 4.3 and 4.4 ).

The results of the comparisons of the wet deposition of the major ions determined from the deeper ADOM cloud and the cloud chemistry model can be generalised as follows. With the exception of case $1+D$ for CLOUD B the wetdeposited amount of sulphate, nitrate and ammonium is always underpredicted. 


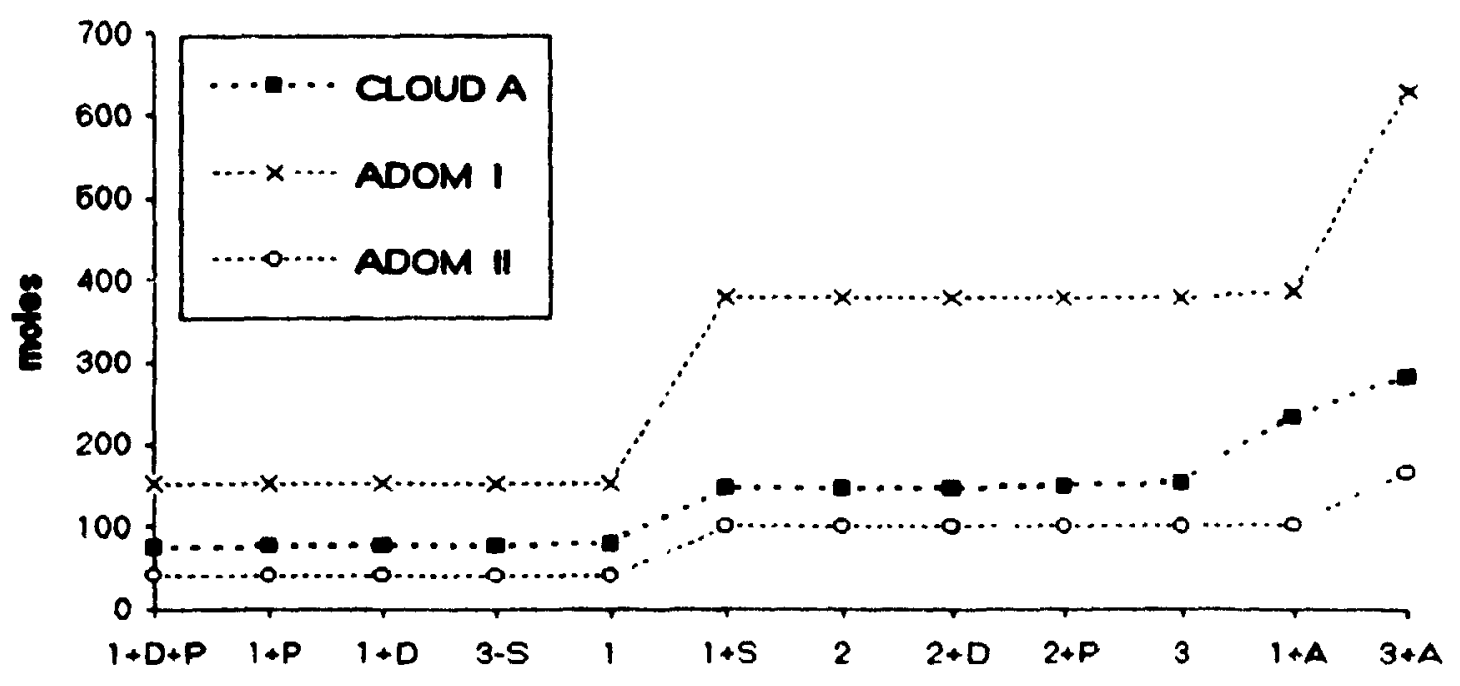

Figure 4.7 Wet deposition of ammonium by CLOUD A and corresponding ADOM clouds

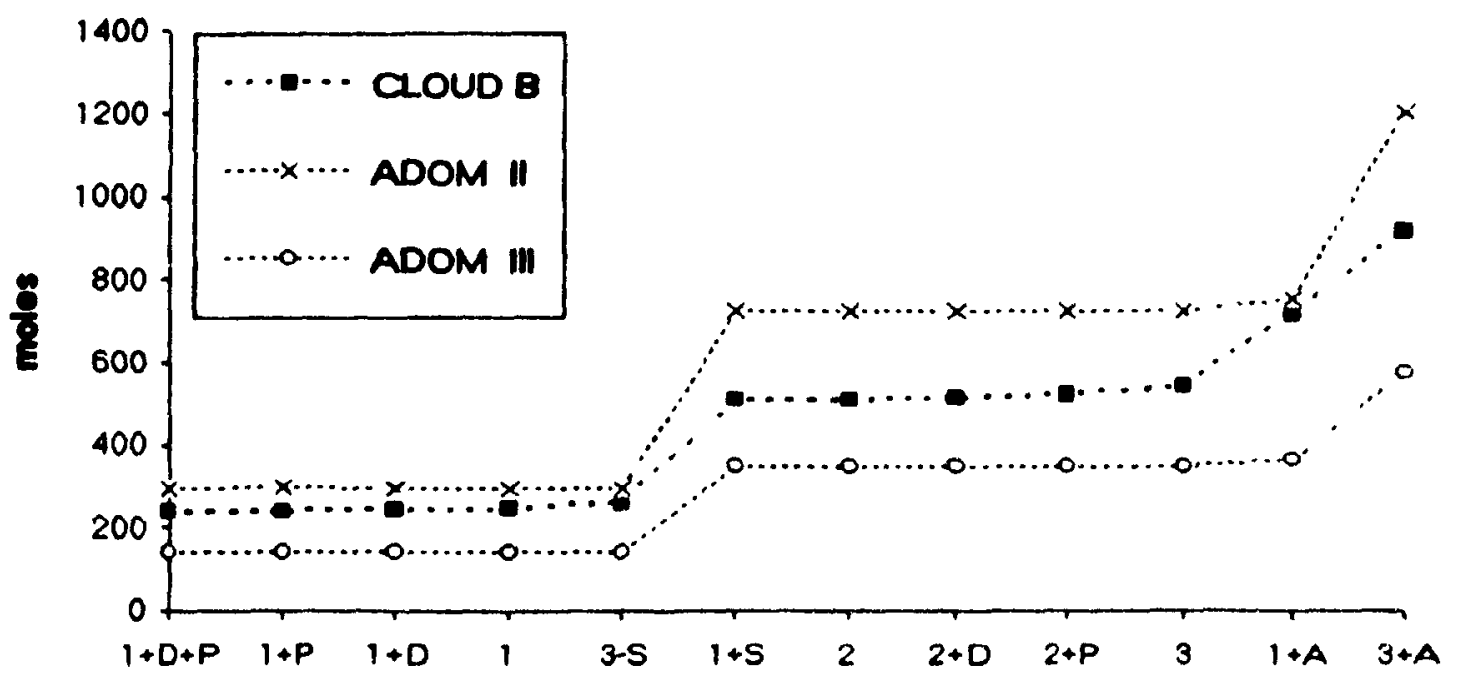

Figure 4.8 Wet deposition of ammonium by CLOUD B and corresponding ADOM clouds 


\section{Rain acidity (pH)}

The ability to adequately predict rain $\mathrm{pH}$ is one of the main requirements that have to be satisfied by any model simulating the processes of wet deposition.

The rain $\mathrm{pH}$ from the cloud chemistry model agrees well with the values from the ADOM scavenging module illustrated in figures 4.9 and 4.10. As usual, the cloud chemistry model simulations are compared first with the results of the deeper ADOM cloud (CLOUD A with ADOM II and CLOUD B with ADOM III). The cases (on $\mathbf{x}$ axis) are ordered according to decreasing values of $\mathrm{pH}$ from the cloud chemistry model simulations.

In almost all of the chemical environments considered, the $\mathrm{pH}$ of the rain is overpredicted by the ADOM scavenging module. The overprediction is generally small. The biggest discrepancies between model results occur for the polluted environments with a relatively high concentration of hydrogen peroxide (case $2+P$ ). The overprediction is then equal to $0.2 \mathrm{pH}$ units. For those situations the underpredictions of the sulphate wet-deposited are among the largest (figures 4.3 and 4.4).

Underprediction of rain $\mathrm{pH}$ by the $\mathrm{ADOM}$ scavenging module occurs for the weakly polluted atmosphere with a relatively high concentration of ammonia (case 
$1+A)$ and, for the bigger cloud, with increased sulphur dioxide (case $1+D$ ). For this latter case the amount of sulphate wet-deposited is overpredicted in the ADOM cloud (this is the only case of sulphate wet deposition overprediction by ADOM), and nitrate and ammonium ion deposition are underpredicted by about equal amounts and hence balance. This accounts for the larger deposition of hydrogen ions in the $\mathrm{ADOM}$ cloud. For the case $1+\mathrm{A}$ the amount of all three major ions that are wetdeposited is underpredicted in the ADOM cloud, but the underprediction of ammonium ions is by far the most important.

The results of the simulations for the shallower ADOM cloud presented in both figures show that the $\mathrm{pH}$ is underpredicted in the shallower cloud by about 0.5 pH units for CLOUD A and by $0.2 \mathrm{pH}$ units for CLOUD B.

The following remark concludes this point: as far as rain acidity is concerned the deeper ADOM cloud performs well and always better than the shallower cloud, obtained by artificial lowering the cloud top location to correspond more closely to the cloud top height from the cloud chemistry model. 


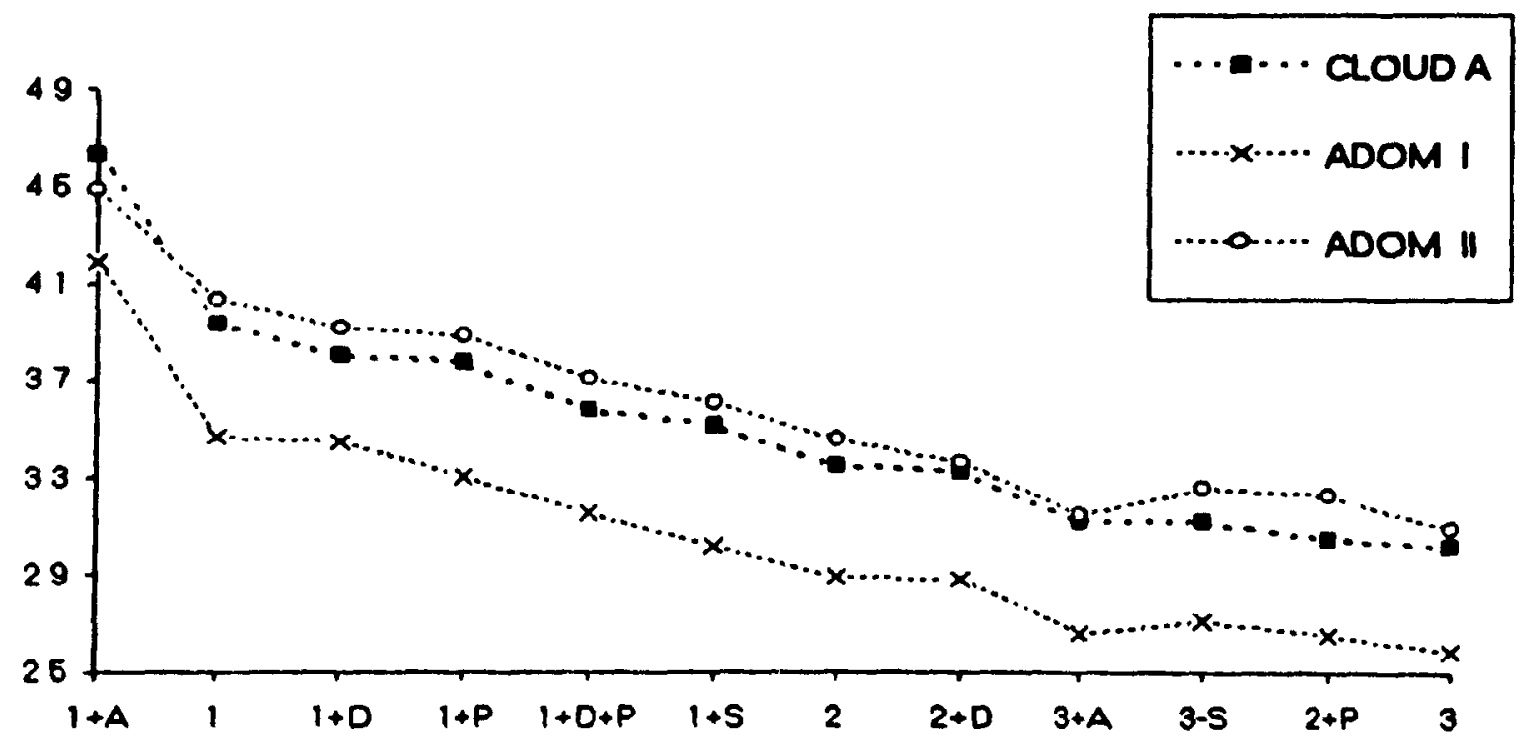

Figure 4.9 Rain acidity for CLOUD A and corresponding ADOM clouds

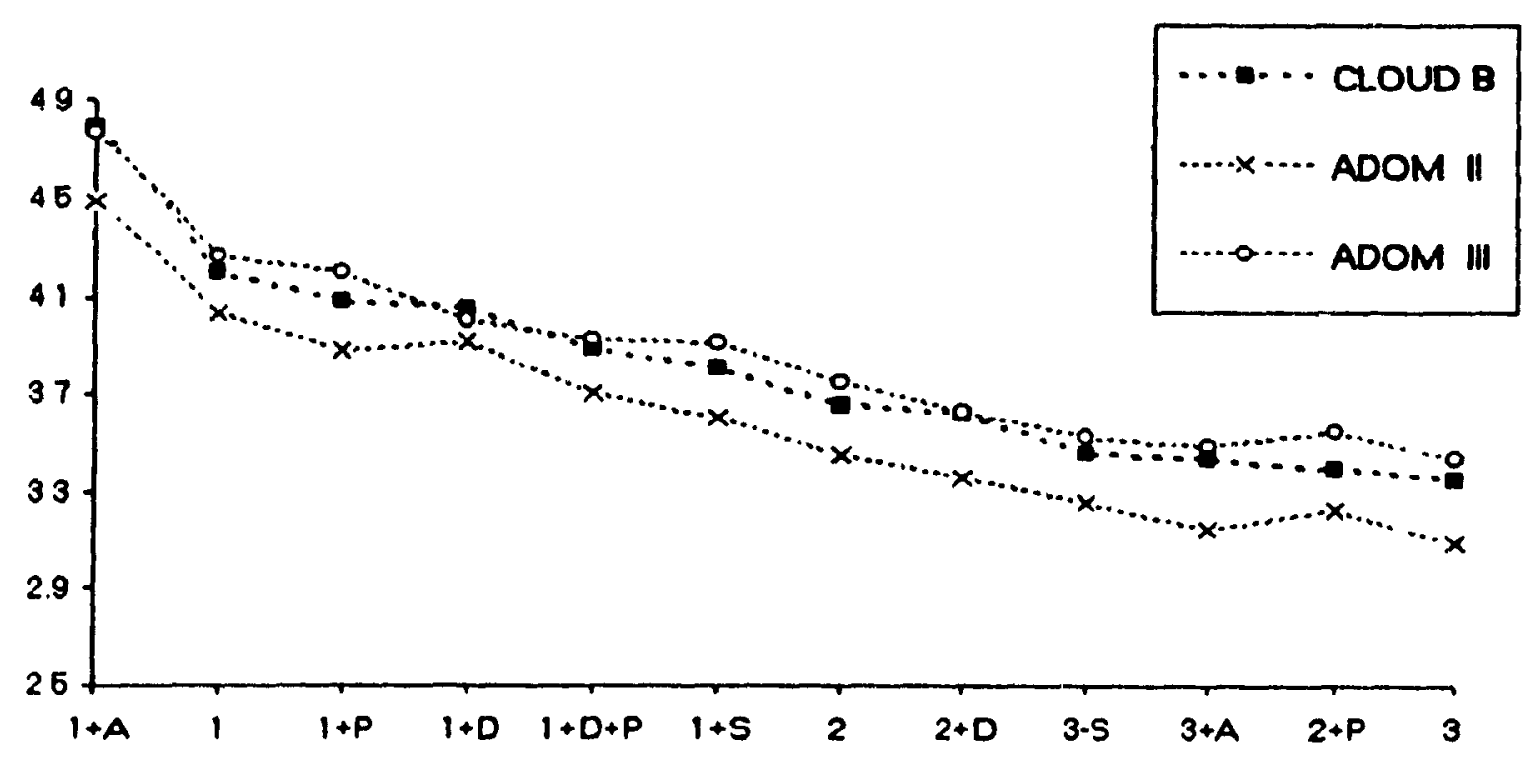

Figure 4.10 Rain acidity for CLOUD B and corresponding ADOM clouds 


\subsection{Wet deposition of hydrogen peroxide}

The wet deposition of hydrogen peroxide is displayed in figures 4.11 and 4.12 . Model predictions agree well for the environments characterised by enhanced concentrations of hydrogen peroxide (case $1+P$ and $2+P$ ) and ammonia (case $1+A$ ). For all remaining situations the amount of wet-deposited hydrogen peroxide simulated by the ADOM scavenging module is negligibly small. It was verified that hydrogen peroxide is depleted from the cloud water within the first few minutes of simulation. In the cloud chemistry model simulation similar depletion is not observed. Typically about five moles of hydrogen peroxide are wet-deposited by CLOUD A and about twenty moles by CLOUD B.

At this point it seems worthwhile mentioning some additional numerical experiments that were performed to investigate the role and importance of the ice phase in the ADOM scavenging module. It was already indicated in section 3.3 that there could be little ice present at the relatively warm cloud top temperature of CLOUD A and CLOUD B. Nevertheless, the wet deposition of hydrogen peroxide in $A D O M$ is very sensitive to the introduction of the ice phase in the model calculations. An important fraction of hydrogen peroxide is trapped in the ice phase during the simulation time step and consequently can not be depleted by oxidation. Part of the trapped hydrogen peroxide is wet-deposited at the end of simulation time step. For all twenty four cases considered an increase in hydrogen peroxide wet 


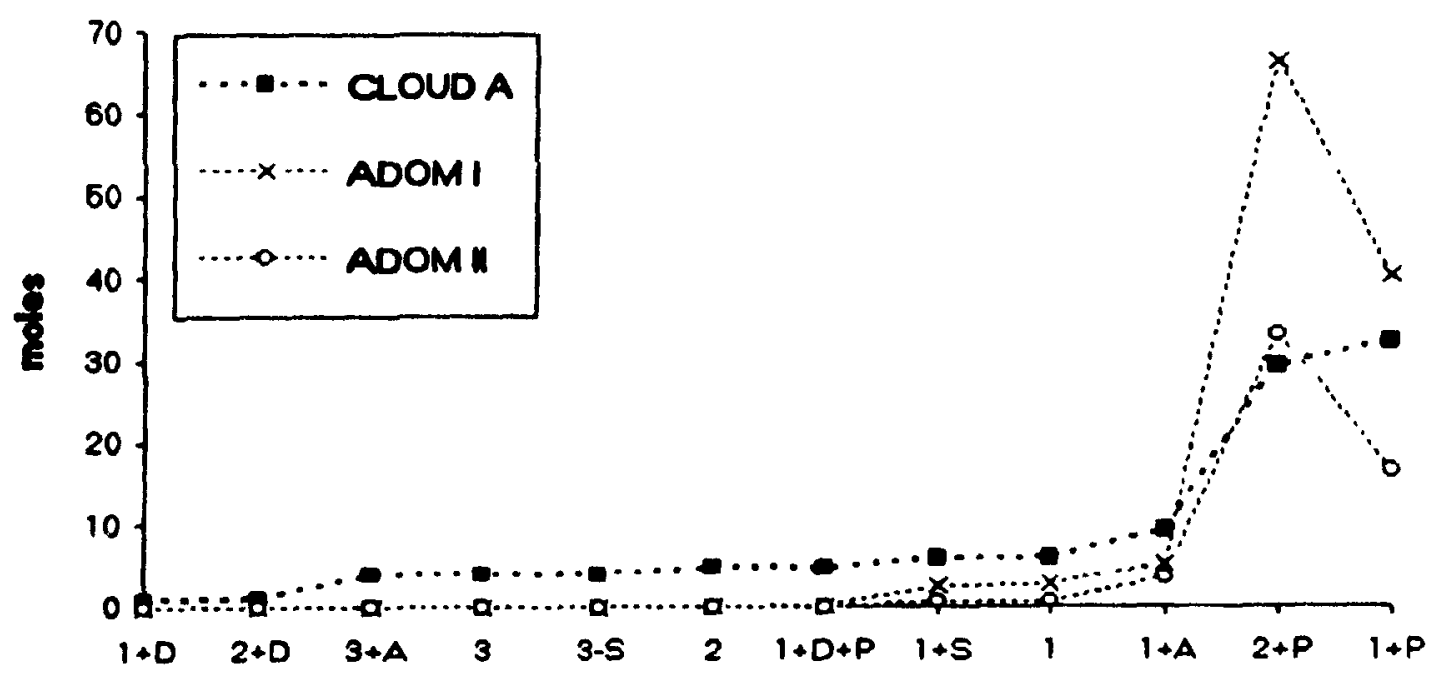

Figure 4.11 Wet deposition of hydrogen peroxide by CLOUD A and corresponding ADOM clouds

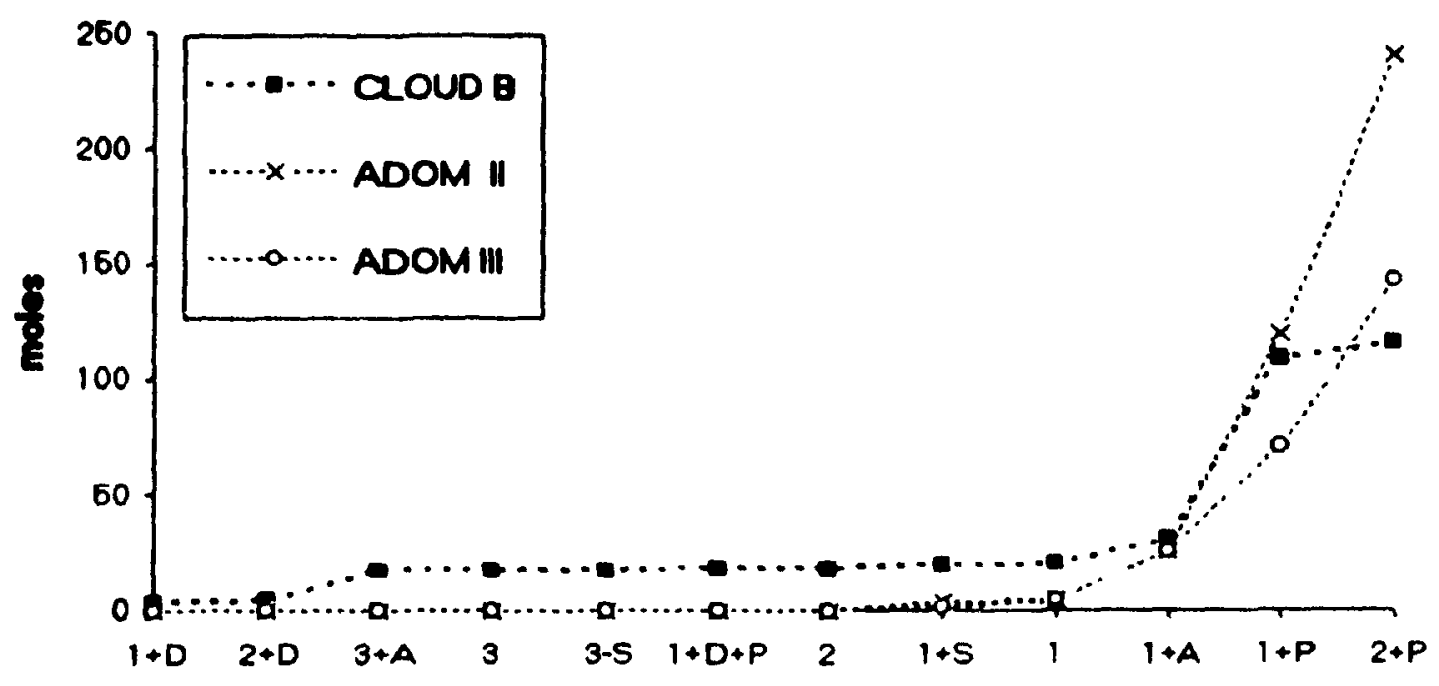

Figure 4.12 Wet deposition of hydrogen peroxide by CLOUD B and corresponding ADOM clouds 
deposition is in fact observed when the ice phase is included in the model. However underprediction of the amount of hydrogen peroxide wet-deposited is still observed for the weakly polluted cases and the smaller cloud (CLOUD A). For the more polluted cases the wet deposition of hydrogen peroxide is significantly overpredicted by the $A D O M$ scavenging module with ice.

\subsection{Total and in-cloud oxidation}

Total oxidation

Total oxidation is a standard cloud chemistry model output. It includes four components: in-cloud oxidation by ozone, in-cloud oxidation ty hydrogen peroxide, in-rain oxidation by ozone and in-rain oxidation by hydrogen peroxide. The value of the variable "total oxidation" is the sum of the number of moles of sulphate from all oxidation processes mentioned above over the simulation time, for the whole domain of the model.

In ADOM total oxidation is not a standard model output, but it may be computed. The oxidation in ADOM occurs only within the cloud, that is there is no division between oxidation in cloud and rain. Total oxidation in the ADOM scavenging module describes the number of moles of sulphate produced by oxidation within the active part of the cloud, during the simulation time step.

The results of the simulations are presented in figures 4.13 and 4.14 . Total oxidation within every ADOM cloud is in general significantly bigger then total 


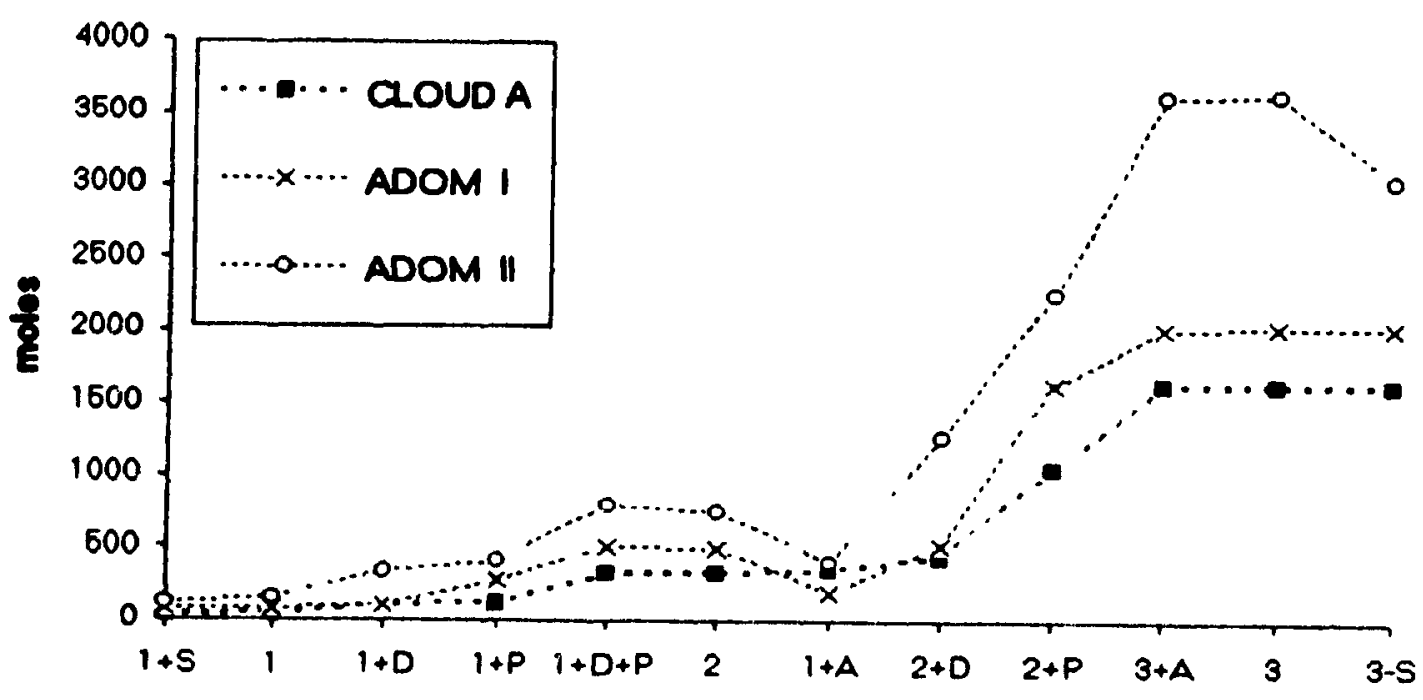

Figure 4.13 Total oxidation for CLOUD A and corresponding ADOM clouds

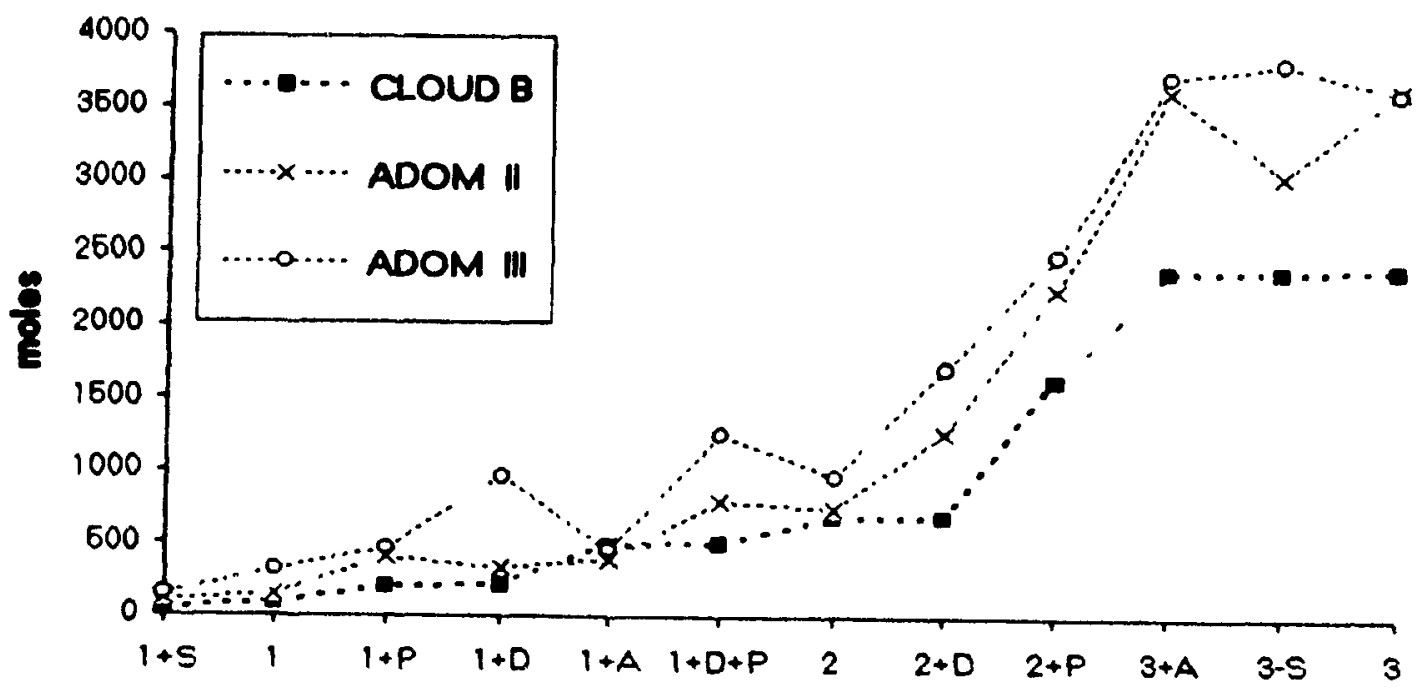

Figure 4.14 Total oxidation for CLOUD B and corresponding ADOM clouds 
oxidation computed by the cloud chemistry model. The shallower ADOM clouds perform better with respect to the values of total oxidation, with the exception of the weakly polluted environment with an enhanced concentration of ammonia (case 1+A). For this environment the total oxidation amount predicted by the deeper ADOM clouds agree well with the total oxidation predicted by the cloud chemistry model. The discrepancies between model predictions for the other environments are of the order of $100 \%$ or more. The total oxidation predicted by the ADOM scavenging module for the weakly polluted environments agrees better with the cloud chemistry model results for CLOUD A, whereas for the polluted environments the agreement is better for CLOUD B.

In-cloud oxidation

In-cloud oxidation fraction is a standard output of ADOM. It is defined as the ratio of amount of the sulphate in the cloud water resulting from in-cloud oxidation to the sum of sulphate in the cloud water from oxidation and nucleation (this later being total sulphate within the cloud in the model). All quantities are cumulative for the simulation time step. The analogous variable may be defined for the cloud chemistry model. In the cloud chemistry model the value of (1) total oxidation within the cloud and rain and (2) total transfer of sulphate from air to cloud and rain water, during the simulation time step, are determined. The variable parallel to the ADOM in-cloud oxidation fraction is defined then as the ratio of (1) to the sum of (1) and (2). The results are presented in figures 4.15 and 4.16 . 


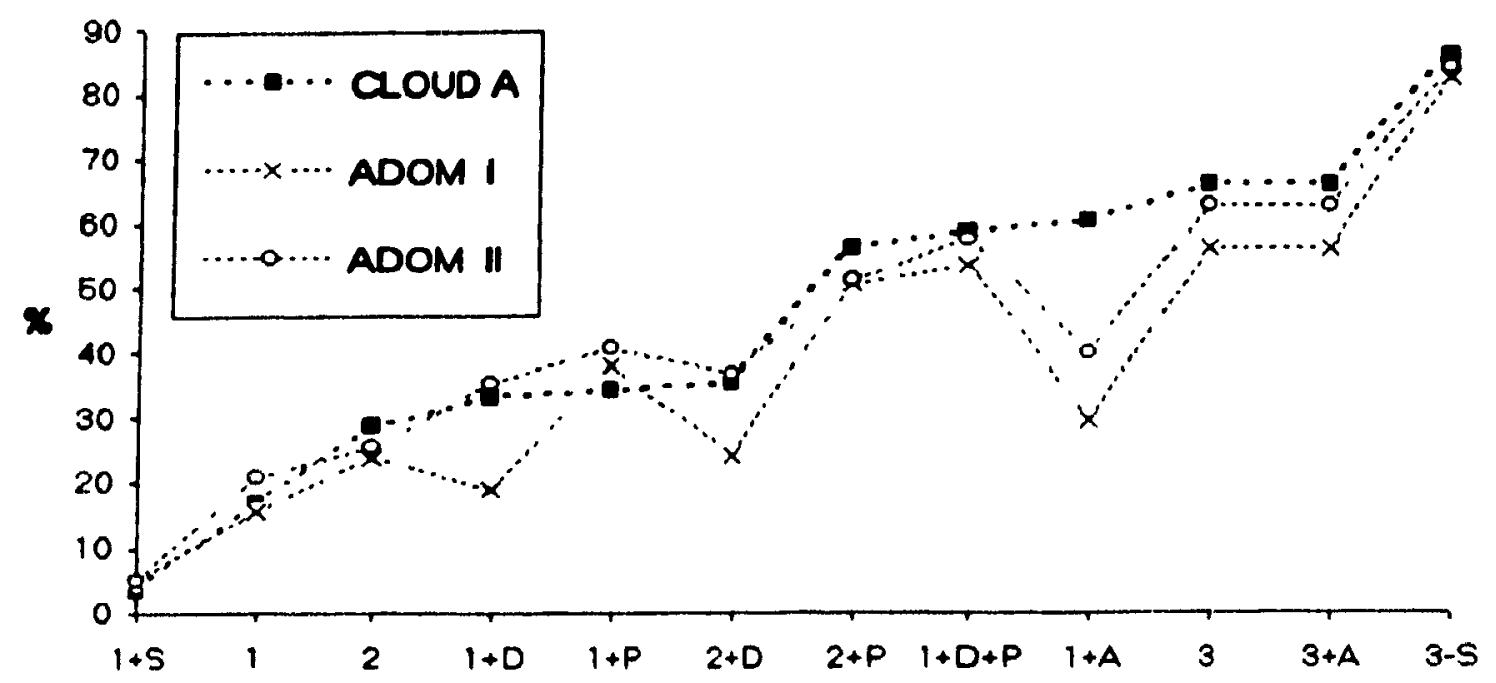

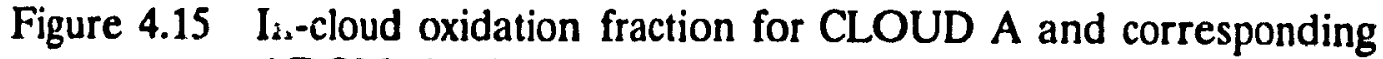
ADOM clouds

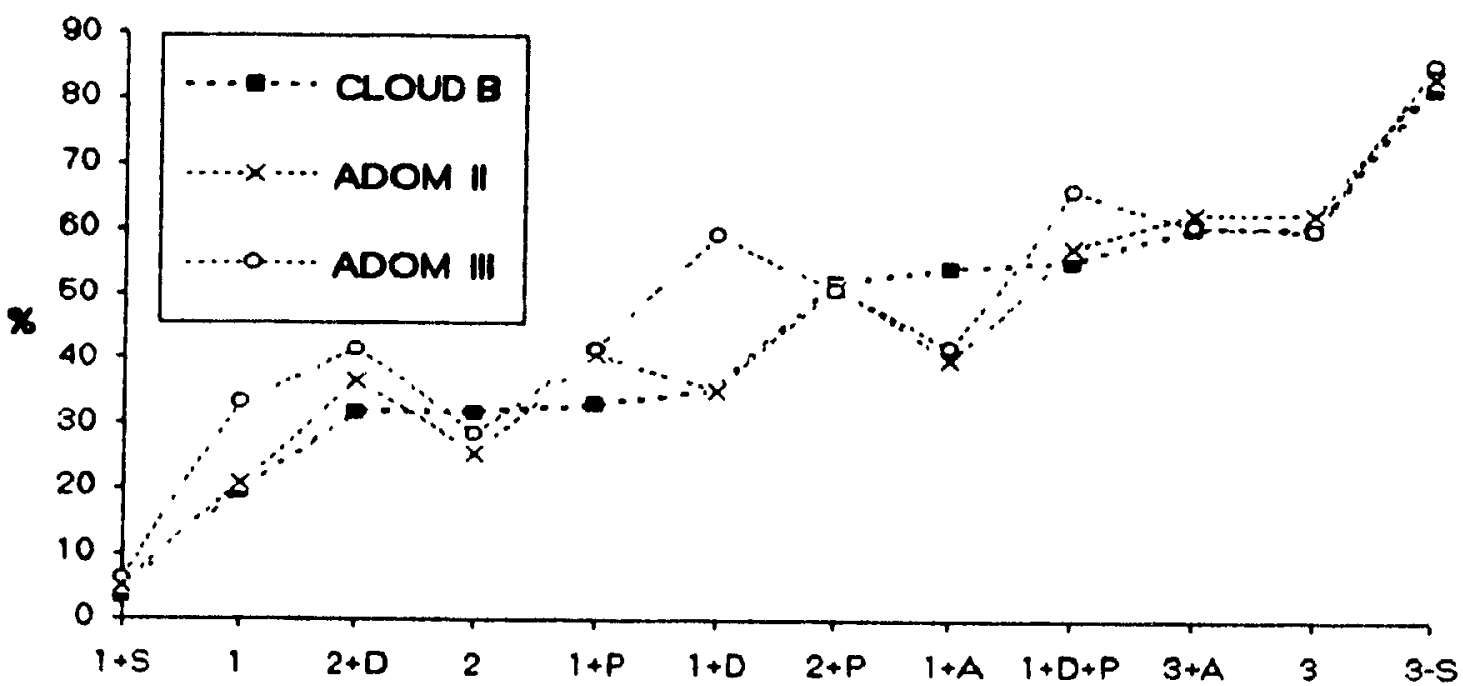

Figure 4.16 In-cloud oxidation fraction for CLOUD B and corresponding ADOM clouds 
For the weakly polluted atmosphere with enhanced concentration of sulphate (case $1+S$ ), nucleation is much more important than oxidation and all three $A D O M$ clouds are characterised by the same value of the in-cloud oxidation fraction. That value, about $5 \%$, agrees well with the cloud chemistry model predictions, which are also almost the same for both CLOUD A and CLOUD B.

For the polluted atmosphere with relatively low concentration of sulphate (case 3-S), oxidation predominates over nucleation scavenging and again the value of in-cloud oxidation fraction for both cloud chemistry model clouds and all three ADOM clouds is almost the same, now about $80 \%$.

For the environments characterised by enhanced concentrations of hydrogen peroxide (cases $1+\mathrm{P}$ and $2+\mathrm{P}$ ) the agreement between model predictions is relatively good, the values differing by less than $7 \%$. For the bigger cloud (CLOUD B) and the higher level of pollution (case $2+\mathrm{P}$ ), exactly the same value of the in-cloud oxidation fraction characterises CLOUD B and the two ADOM clouds (II and III).

The atmospheres with enhanced concentrations of sulphur dioxide (cases 1+D and $2+D$ ) are characterised $\mathrm{b}$, the biggest differences between in-cioud oxidation values for different ADOM clouds, but the CLOUD A and ADOM II predictions agree well. The biggest discrepancy between model predictions, exceeding $20 \%$, occurs for CLOUD B and the less polluted environment (case 1+D). For the more 
polluted environment (case $2+D$ ) this discrepancy is about $10 \%$.

For the weakly polluted atmosphere with enhanced concentration of ammonia (case $1+\mathrm{A}$ ) the discrepancies between model predictions are important: about $20 \%$ for CLOUD A and 15\% for CLOUD B. For the high pollution levels with enhanced concentration of ammonia (case $3+A$ ) the differences between model predictions are less than $5 \%$.

The comparison of the values of the in-cloud oxidation fraction determined from both models can be summarised as follows. For the dynamic simulation that produces CLOUD A (figure 4.15) the agreement between model predictions is good. The discrepancies between model predictions (CLOUD A and ADOM II) are below $5 \%$, with the exception of the weakly polluted environment with enhanced ammonia (case $1+A$ ) when this discrepancy is about $20 \%$. The agreement between model predictions for the second dynamic simulation (figure 4.16) is less good especially for the weakly polluted atmosphere.

\subsection{Fractional acidity}

Fractional acidity f, following Schwartz (1984), is expressed in terms of variables that can be directly measured:

$$
\mathrm{f}=\left[\mathrm{H}^{+}\right] /\left(\left[\mathrm{NO}^{-}\right]+2\left[\mathrm{SO}^{=}\right]\right)
$$


The aqueous concentration of all three species in the definition of the fractional acidity are underpredicted in ADOM. However, fractional acidity is overpredicted for all chemical environments considered for both CLOUD $A$ and CLOUD B (figures 4.17 and 4.18). The discrepancies between model predictions are rather small, the biggest differences occur for the weakly polluted atmospheres. The shallower ADOM cloud better predicts the values of fractional acidity in every case. 


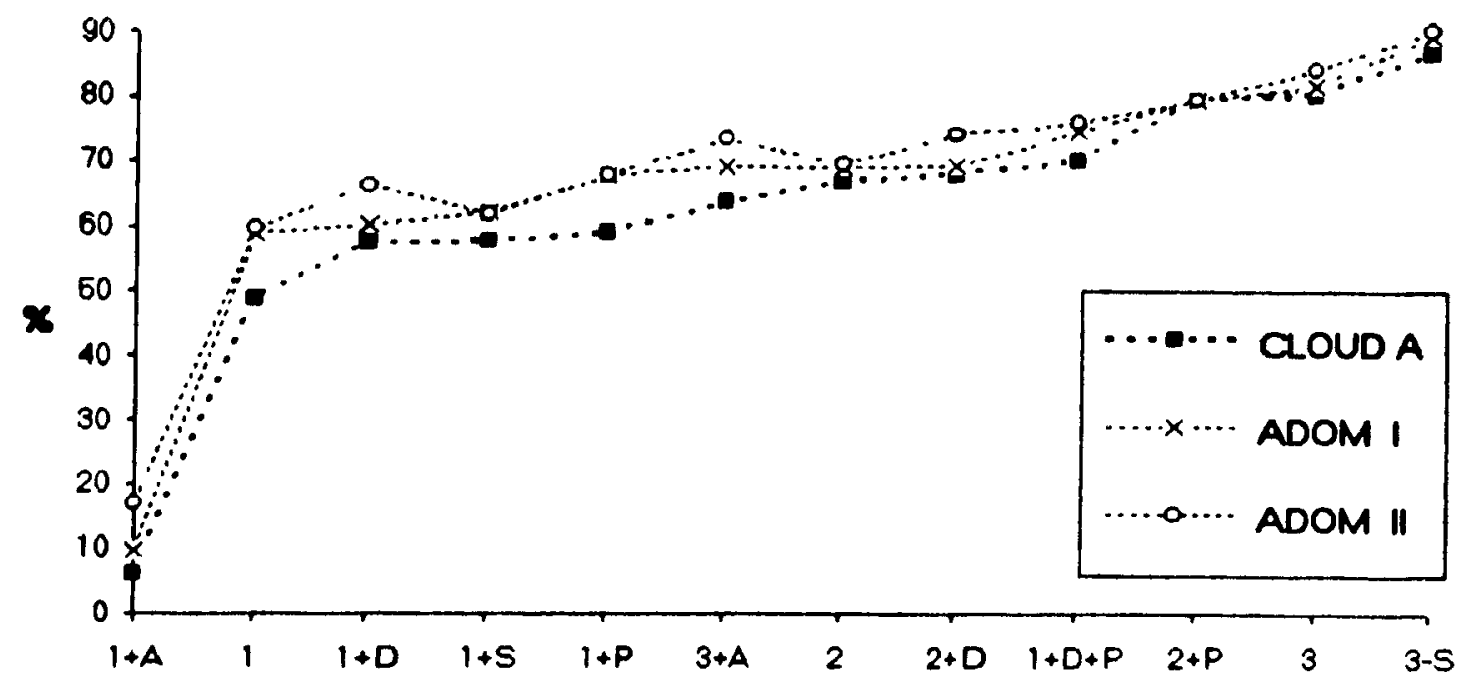

Figure 4.17 Fractional acidity for CLOUD A and corresponding ADOM clouds

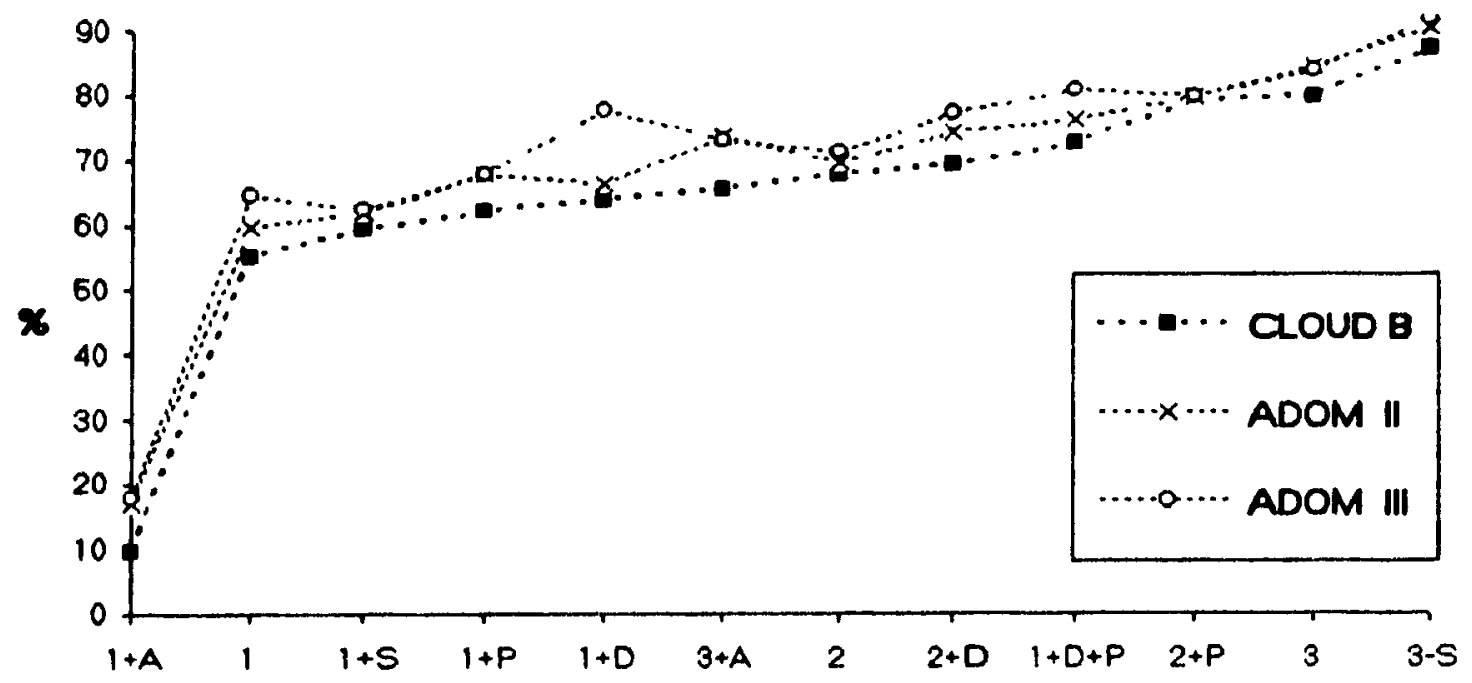

Figure 4.18 Fractional acidity for CLOUD B and corresponding ADOM clouds 


\subsection{Concentration of chemicals}

Concentrations of the species in the air are provided by both models but with different spatial resolution. Initial concentration profiles are given as mean values for every level for each model. At the very end of the simulation time step ADOM yields the final concentration profiles also as mean values for each level. The threedimensional cloud chemistry model follows the evolution of concentrations in the cloud and rain water as well as in the air for each grid square of the model. This detailed output can not be compared with the ADOM results. Only mean values for levels, interpolated because the vertical resolution of models is not the same, can be compared.

The wet deposition resulting from both models is only a very small fraction of the initial amount of chemicals available in the domain. As the initial mean concentration profiles were almost identical, the only slightly modified final mean concentration profiles also have to be similar, and they are. The details of the final air concentration profiles do not seem to be worth analyzing but two important features will be mentioned.

As a cloud grows and dissipates it redistributes chemical species in the vertical. The environmental concentration profiles are modified by cloud, primarily in the immediate vicinity of the cloud. The ADOM clouds extend much higher than corresponding cloud chemistry model clouds. Consequently important differences between model predictions can arise above the cloud chemistry model top heights. 
These discrepancies between model predictions although small in absolute value can have important consequences for the LRTAP models. As wind velocity increases with height even small amounts of chemicals transported up by clouds can subsequently propagate far from the cloud location. The additional concentration related to incorrectly simulated cloud top heights can influence remote regions.

The change in the sulphate in the air due to the cloud is the same as the difference between the sulphate produced by oxidation and the sulphate that is wetdeposited. This difference is shown in figures 4.19 and 4.20. There exists a large difference between model predictions especially for high levels of pollution, with the exception of the weakly polluted atmosphere with enhanced concentration of ammonia (case 1+A). Although the final concentrations of sulphate in the air predicted by both models are similar the net amounts of sulphate produced within the clouds are significantly different. 


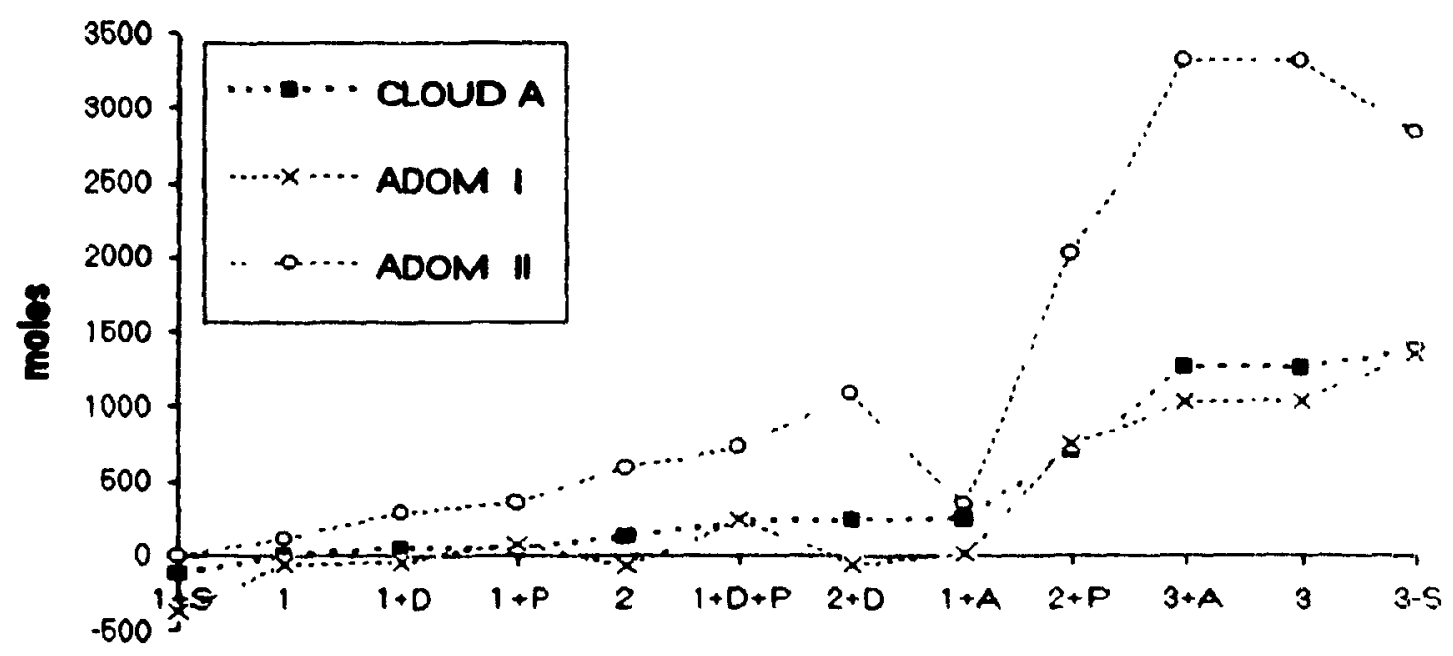

Figure 4.19 Difference between final and initial sulphate in the air for CLOUD $A$ and corresponding ADOM clouds

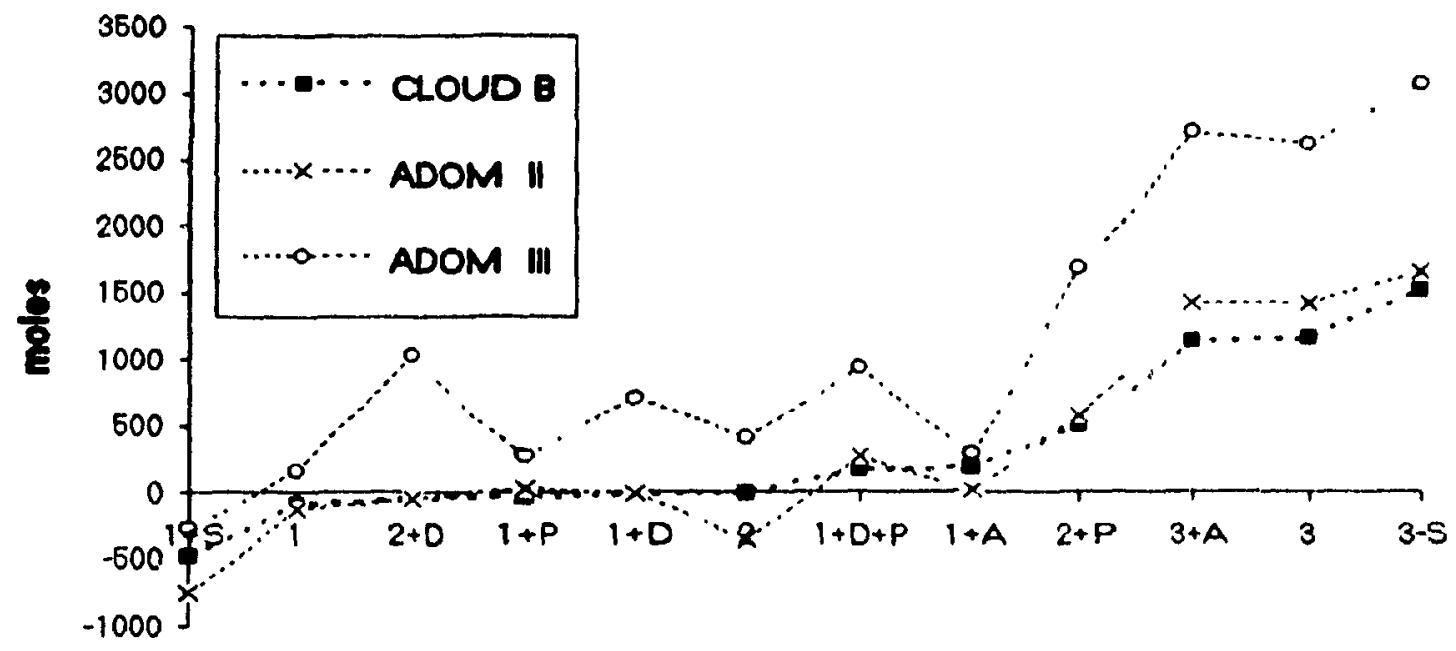

Figure 4.20 Difference between final and initial sulphate in the air for CLOUD B and corresponding ADOM clouds 


\subsection{Comparison of model results for different ADOM clouds}

Up to now, in this chapter inter-model comparisons were performed, but this section is concerned only with ADOM clouds. The characteristics of the simulated ADOM clouds: ADOM I, ADOM II and ADOM III have already been described in section 3.3.2 and recapitulated in table 3.3. A few remarks about the comparison of the ADOM scavenging module output generated for different ADOM clouds will be presented below.

The values of total oxidation, in-cloud oxidation fraction, rain acidity and fractional acidity are independent of assumed precipitation rate for a particular ADOM cloud. This implies that whether ADOM II represents CLOUD A or CLOUD B is not important, both are characterised by the same values of the above four variables. The amount of chemicals wet-deposited by ADOM clouds depends on precipitation rate and consequently is different for ADOM II representing CLOUD $A$ and ADOM II representing CLOUD B.

Total oxidation, that is the total number of moles of sulphate resulting from oxidation, increases with ADOM cloud top height. This occurs for each chemical environment. The same property holds for the total number of moles of sulphate that is scavenged by the cloud, ADOM III scavenges more than ADOM II and ADOM II more than ADOM I. The total sulphate scavenged can be determined because both in-cloud oxidation fraction and total oxidation are known.

In-cloud oxidation fraction also increases with cloud volume with the exception 
of the highly polluted environment (case 3 ) and highly polluted environment with increased concentration of ammonia (case $3+A$ ). For these two situations in-cloud oxidation fraction is the largest for ADOM Il. Total oxidation for these two cases is almost the same within ADOM II and ADOM III, ADOM III scavenges more sulphate than ADOM II and consequently in-cloud oxidation fraction is larger for ADOM II.

In-cloud oxidation fraction is above $50 \%$ for the highly polluted environments (cases $3,3+\mathrm{A}$ and $3-\mathrm{S}$ ) and for the weakly polluted environment with enhanced concentrations of both sulphur dioxide and hydrogen peroxide (case $1+P+D)$. For the remaining cases nucleation scavenging is more important than oxidation within the cloud (in-cloud oxidation is below 50\%) and this occurs for all three ADOM clouds for all chemical environments with the exception of the weakly polluted atmosphere with enhanced concentration of sulphur dioxide (case 1+D). This case is characterised by the biggest differences between the values of in-cloud oxidation fraction for different $\mathrm{ADOM}$ clouds (in-cloud oxidation fraction is equal about $15 \%$ for $\mathrm{ADOM} I, 35 \%$ for $\mathrm{ADOM}$ II and $60 \%$ for $\mathrm{ADOM}$ III).

Fractional acidity also increases with the volume of ADOM cloud. The only exception occurs for two chemical environments, previously mentioned (cases 3 and $3+A)$, when in-cloud oxidation fraction, similarly as now fractional acidity, is bigger for ADOM II than for ADOM III.

Rain acidity is the smallest for ADOM III and the largest for ADOM I for every chemical environment. Even though both sulphate scavenging and total 
oxidation are the largest for the deepest ADOM cloud its $\mathrm{pH}$ is relatively high. This property is due to the increase of liquid water content with the height of the top of the ADOM cloud (table 3.3).

The increase of cloud water $\mathrm{pH}$ (or equivalently decrease of hydrogen ion concentration) with the increase of cloud top height is consistent with the corresponding decrease in the amount of chemicals wet-deposited. Now only two ADOM clouds can be compared simultaneously ADOM I with ADOM II both representing CLOUD A, and ADOM II with ADOM III both representing CLOUD B. The amount of chemicals wet-deposited by the shallower ADOM clouds, presented in section 4.2 and in figures $4.1,4.2$ and 4.5 to 4.8 , are always significantly bigger than the amount of chemicals wet-deposited by the deeper clouds. This implies that concentrations of species are higher in the shallower clouds. This is understandable in that the shallower ADOM clouds are created by the air parcels originating from lower, more polluted levels. At the same time liquid water content of the shallower ADOM cloud is smaller than the liquid water content of deeper cloud. This property also promotes higher concentrations of chemicals in cloud water in the shallower cloud. Total oxidation as well as total scavenged sulphate may be higher in the deeper cloud because of its greater cloud volume. 


\section{Chapter 5}

\section{SUMMARY AND CONCLUSIONS}

\subsection{Summary}

It is well recognised that clouds have significant effects on long-range transport of air pollutants. Both chemical transformations within the liquid phase and redistribution of pollutants due to air motions related to cloud evolution are important. Consequently, the cloud module must be one of the critical parts of any LRTAP model. There is no doubt that clouds are complicated three dimensional structures, evolving in time and space with mutually interacting microphysics and dynamics. Their detailed simulation within huge LRTAP models is unfeasible because of computational constraints. The cloud module has to be simple and the ADOM scavenging module satisfies this requirement. But is this simplification acceptable in terms of model performance? Are model predictions reliable within reasonable limits? Does the $A D O M$ scavenging module need to be changed or improved and how? To answer these types of questions model evaluation is necessary.

As all this is about real processes the answer should be given on the basis of 
field experiments. But taking into consideration all the problems related to gathering adequate data, the complementary approach, inter-model comparisons, is proposed within this study. A three-dimensional cloud chemistry model is used to evaluate the ADOM scavenging module performance. Results obtained from both models are compared for equivalent initial conditions. We believe that the three-dimensional cloud chemistry model dynamics and microphysics are sufficiently complex to represent important features of evolving convective cloud. This belief is supported by several previous successful applications of this model. The chemistry parts of both the ADOM scavenging module and the cloud chemistry model are well founded and it can be assumed that the reactions included adequately describe the important chemical processes. With these assumptions in mind we hope to obtain insight into the question of how the simplifications of dynamics and microphysics in the ADOM scavenging module influence the output. Inter-model comparisons allow more systematic studies of analyzed model properties and are much simpler to carry than comparisons with observational data, but are not free from the influence of approximations introduced by the other model. Consequently the conclusions have to be formulated prudently and a dose of scepticism should accompany this type of study.

Both models applied in this study predict the amounts of chemicals wetdeposited by precipitating cloud, estimate the final concentration of species in the air, give some additional information about oxidation processes in terms of total number of moles of sulphate that result from oxidation, and allow the comparison of the 
relative importance of oxidation and nucleation. These elements of output were compared for two different clouds simulated by the cloud chemistry model (CLOUD A and CLOUD B, where CLOUD B is the bigger cloud) and then by the ADOM scavenging module. These clouds evolved in twelve different environments each, characterising different levels of atmospheric pollution.

\subsection{Conclusions}

The wet-deposited amount of sulphate and ammonium ions predicted by both models agree well. A systematic small underprediction of these quantities by the ADOM scavenging module was observed for all of the chemical environments considered. The largest discrepancies between model predictions of wet deposition of sulphate and ammonium ions were observed for the relatively clean atmosphere with enhanced initial concentrations of ammonia. The $\mathrm{pH}$ of the rain determined by the cloud chemistry model and the ADOM scavenging module were also in a good agreement but with a tendency for overprediction by the ADOM scavenging module. The discrepancies between predicted rain acidity were more important for the environments with relatively high initial concentration of hydrogen peroxide. The wet deposition of nitrate was significantly underpredicted by the ADOM scavenging module. All these properties characterised both dynamical cases. With respecî to the elements presented above, the $\mathrm{ADOM}$ scavenging module performance did not change significantly with the chemical properties of the environment. The same could 
be stated with the respect to the dynamical properties of the environment but as only two different clouds were simulated this conclusion is very tentative. A more reliable statement may be formulated only after analysis of more clouds developing in dynamically and therizodynamically different environments.

The amount of hydrogen peroxide wet-deposited by rain was significantly smaller in the ADOM scavenging module than in the cloud chemistry model for the majority of chemical environments. Model predictions agree well only for the weakly polluted atmosphere with enhanced concentration of ammonia and for the environments with enhanced concentrations of hydrogen peroxide.

The total oxidation was significantly overpredicted by the ADOM scaverging module for all environments with the exception of the weakly polluted atmosphere with increased concentration of ammonia when the agreement was good.

The in-cioud oxidation fraction for the smallest cloud (CLOUD A) is better represented by the in-cloud oxidation fraction for $A D O M$ cloud than in-cloud oxidation fraction for the biggest cloud (CLOUD B) by the corresponding values for ADOM cloud. There exist, however, properties common to both CLOUD A and CLOUD B: model predictions of in-cloud oxidation fraction value agree well for all environments in which either oxidation or nucleation scavenging dominates, and the biggest discrepancies of model predictions occur for the weakly polluted atmospheres 
with enhanced conceniration of ammonia.

The final concentration of chemicals in the air was described in the ADOM scavenging module in terms of mean values for each vertical level of the model averaged over the whole domain. Since the cloud has only a small influence on the vertical distributions when averaged over the whole domain, the differences between model predictions are small. The averaged final concentration profiles extended higher up in the ADOM scavenging module than in the cloud chemistry simulations because of significantly larger cloud top heights in ADOM.

Along with the inter-model comparison experiments, the sensitivity of the ADOM scavenging module to the cloud top location was investigated.

Cloud top location is an optional input parameter in ADOM. ADOM has the general tendency to locate cloud top higher than the specified input value. The clouds simulated by the ADOM scavenging module, ADOM II and ADOM III, extended significantly higher than the respective cloud chemistry model clouds, CLOUD A and CLOUD B.

The shallower clouds simulated in this study, ADOM I to represent CLOUD A and ADOM II to represent CLOUD B, were obtained by decreasing the input value of the cloud top heights. Although input values of ADOM cloud top heights were lower than the three dimensional cloud chemistry model cloud top heights, the resulting ADOM cloud top heights were now closer to the simulated cloud chemistry 
model cloud top heights. The amounts of the various species wet-deposited by the shallower ADOM clouds were significantly increased as well as the rain acidity if cumpared with the results for the deeper ADOM clouds. The shallower ADOM clouds pertormed in general less well than deeper ADOM clouds. The shallower ADOM clouds, however, better represented nitrate wet deposition and total oxidation (which was smaller but still too big), and sometimes also in-cloud oxidation fraction and ammonium wet deposition.

\subsection{Suggestion for future studies}

For the particular dynamical properties of the environment investigated in this study the ADOM scavenging module results agree generally reasonably well with the cloud chemistry model results for the different intensities of atmospheric pollution. It would be interesting to evaluate the ADOM scavenging module for other dynamical conditions allowing for instance the occurrence of multiple cloud cycles. The simulation of bigger, longer lasting cloud that produce more rain might yield more information about ADOM perf srmance. More experiments would provide an answer to the question: is the agreement of model results found in this study due to chance or is this a general property of ADOM. 


\section{LIST OF REFERENCES}

Berkowitz, C.M., 1991: The response of sulfate linearity and precipitation chemistry to gasphase profiles. Almos. Environ., 25A, 1295-1311.

Berkowitz, C.M., R.C. Easter and B.C. Scott, 1989: Theory and results from a quasi-steadystate precipitation-scavenging model. Atmos. Environ., 23, 1555-1571.

Carmichael, G.R., L.K. Peters and R.D. Saylor, 1991: The STEM-II regional scale acid deposition and photochemical oxidant model - I. An overview of model development and applications. Atmos. Environ., 25A, 2077-2090.

Carmichael, G.R. and L.K. Peters, 1984: An Eulerian transport/transformation/removal model for $\mathrm{SO}_{2}$ and sulfate - I. Model development. Atmos. Environ., 18, 937-951.

Carmichael, G.R. and L.K. Peters, 1984: An Eulerian transport/transformation/removal model for $\mathrm{SO}_{2}$ and sulfate - II. Model calculation of $\mathrm{SO}_{x}$ transport in the eastern united States. Atmos. Environ., 18, 953-967.

Chang, J.S., R.A. Brost, I.S.A. Isaksen, S. Madronich, P. Middleton, W.R. Stockwell and C.J. Walcek, 1987: A three-dimensional Eulerian acid deposition model: physical concepts and formulation. J. Geophys. Res., 92, 14681-14700.

Daum, P.H., T.J. Kelly, R.L. Tanner, X. Tang, K. Anlauf, J. Bottenheim, K.A. Brice and H.A. Wiebe, 1989: Wirter measurements of trace gas and aerosol composition at a rural site in southern Ontario. Atmos. Environ. 23, 161-173.

Dollard, G.J., B.M.R. Jones and T.J. Davies, 1991: Measurements of gaseous hydrogen peroxide and PAN in rural southern England. Atmos. Environ., 25A, 2039-2053.

Easter, R.C., M.T. Dana, J.M. Thorp, K.M. Busness, J.M. Hales, G.S. Raynor, C.M. Benkovitz, R.L. Tanner and J.D. Shannon, 1984: Overview of the Oxidation and Scavenging Characteristics of April Rains (OSCAR) experiment. PNL-4869, Pacific Northwest Laboratory, Richland, WA.

Easter, R.C. and J.M. Hales, 1984: PLUVIUS: A generalised one-dimensional model of reactive pollutant behavior, including dry deposition, precipitation formation and wet removal. EPRI Report.

Fuchs, N.A. and A.G. Sutugin, 1971: High-dispersed aerosols. In: International Reviews of Aerosol Physics and Chemistry, Vol. 2, G.M. Hidy and J.R. Brock, eds., Pergamon Press, New-York, pp. 1-60. 
Giles, A., 1987: The influence of the ice phase on the simulated chemistry of a rainband. M.Sc. thesis, Dept. of Meteorology, McGill University, $118 \mathrm{pp}$.

Hansen, D.A., K.J. Puckett, J.J. Jansen, M. Lusis and J.S. Vickery, 1991: The Eulerian Model Evaluation Field Study (EMEFS). Preprints, Seventh Joint Conf. on Applications of Air Pollution Meteorology with AWMA, 14-18 January 1991, New Orleans, LA., Am. Met. Soc., Boston, Mass., pp. 58-62.

Hill, F.B. and R.F. Adamowicz, 1977: A model for rain composition and the washout of sulfur dioxide. Atmos. Environ., 11, 917-927.

Iribarne, J.V. and H.R. Cho, 1989: Models of cloud chemistry. Tellus, 41 B, 2-23.

Isaac, G.A., W.R. Leaitch and J.W. Strapp, 1990: The vertical distribution of aerosols and acid related compounds in arr and cloud water. Almos. Environ., 24A, 3033-3046.

Karamchandani, P.K., A. Venkatram, 1992: The role of non-precipitating clouds in producing ambient sulfate during summer: results from simulations with the Acid Depositon and Oxidant Model (ADOM). Atmos. Environ., 26A, 1041-1052.

Kelly, T.J., S.E. McLaren and J.A. Kadlecek, 1989: Seasonal varıations in atmospheric $\mathrm{SO}_{x}$ and $\mathrm{NO}_{\mathrm{y}}$ species in the Adirondacks. Atmos. Environ., 23, 1315-1332.

Kessler, E., 1969: On the distribution and continuity of water substance in atmospheric circulations. Met. Monographs, 10, no. 32, Am. Met. Soc., 84 pp.

Leaitch, W.R., J.W. Strapp, G.A. Isaac, H.A. Wiebe and K.G. Anlauf, 1991: Airborne filter intercomparisons with cloud water and ground-level filter measurements. Proceedings of the Airborne Aerosol Inlet Workshop, Boulder, CO, 21 pp.

Leighton, H.G., M.K. Yau, A.M. Macdonald, J.S. Pitre and A. Giles, 1990: A numerical simulation of the chemistry of a rainband. Atmos. Environl., 24A, 1211-1217.

Levine, S.Z. and S.E. Schwartz, 1982: In-cloud and below cloud scavenging of nitric acid vapour. Atmos. Environ., 15, 1725-1734.

Meagher, J.F., K.J. Olszyna, F.P. Weatherford and V.A. Mohnen, 1990: The avalability of $\mathrm{H}_{2} \mathrm{O}_{2}$ and $\mathrm{O}_{3}$ for aqueous phase oxidation of $\mathrm{SO}_{2}$. The questions of linearity. Atmos. Environ., 24A, 1825.1829.

Pitre, J.S., 1986: The role of oxidation in the chemistry of a rainband. M.Sc. thesis, Dept. of Meteorology, McGill University, 115 pp. 
Raymond, D.J. and A.M. Blyth, 1986: A stochastic mixing model for nonprecipitating cumulus clouds. J. Atmos. Sci., 43, 2708-2718.

Schwartz, S.E., 1984: Both sides now: the chemistry of clouds. Environmental Sciences. F.S. Sterett, ed., pp. 89-144, Annals New York Acad. Sci., 502.

Schwartz, S.E., 1986: Mass-transport considerations pertinent to aqueous-phase reactions of gases in liquid water clouds. In: Chemistry of Multiphase Atmospheric Systems. W. Jaeschke, ed., NATO ASI Series Vol G6, Springer-Verlag, Rerlin, pp. 415-471.

Scott, B.C., 1978: Parametrization of sulphate removal by precipitation. J. Appl. Met., 10, 1375-1389.

Seinfeld, J.H. 1986: Atmospheric Chemistry and Physics of Air Pollution. John Willey and Sons. New York, 738 pp.

Steiner, J.T., 1973: A three-dimensional model of cumulus cloud development. J. Atmos. Sci., 30, 414-435.

Strapp, J.W., W.R. Leaitch, K.G. Anlauf, J.W. Bottenheim, P. Joe, R.S. Schemenauer, H.A. Wiebe, G.A. Isaac, T.J. Kelly and P.H. Daum, 1988: Winter cloud water and air composition in central Ontar:o. J. Geophys. Res., 93, 3760-3772.

Tremblay, A., 1987: Cumulus cloud transport, scavenging and chemistry: observations and simulations. Atmos. Environ., 21, 2345-2364.

Tremblay, A., 1985: A three dimensional cloud chemistry model. Ph.D. thesis, Dept. of Meteorology, McGill University, 129 pp.

Tremblay, A. and H.G. Leighton, 1986: A three-dimensional cloud chemistry model. J. Clim. Appl. Met., 25, 652-671.

Venkatram, A., 1986: Development of a model for the transport and deposition of acidifying pollutants. ERT Doc No 10402569101,80 pp.

Venkatram, A. and P.K. Karamchandani, 1988: The ADOM II scavenging module. ERT Document No 0780-004-205, Pacific Northwest Laboratory, Richland, :WA.

Venkatram, A. and P.K. Karamchandani, 1986: Source-receptor relationships. A look at acid deposition modeling. Environ. Sci. Technol, 20, 1084-1091.

Venkatram, A., P.K. Karamchandani and P.K. Misra, 1988: Testing a comprehensive acid deposition model. Atmos. Environ., 22, 737-747. 
Walcek, C.J. and G.R. Taylor, 1986: A theoretical method for computing vertical distributions of acidity and sulfate production within cumulus clouds. J. Aimos. Sci., 43, 339-355.

Yau, M.K., 1981: Documentation of the computer program CU-3D for the three dimensional single-cloud model. CSIR Special Report SWISK 26, Pretoria, South Africa, 33 pp.

Yau, M.K. and S. Macpherson, 1984: Numerical simulation of an Alberta thunderstorm. Proc. 9th Int. Cloud Physics Conference, Tallinn, USSR, 585-588.

Yau, M.K. and R. Michaud, 1982: Numerical simulation of a cumulus ensemble in three dimensions. J. Atmos. Sci., 39, 1062-1079.

Young, J.R. and F.W. Lurmann, 1984. ADOM/TADAP model development program, Vol.7: Aqueous-phase chemistry. ERT Document No P-B980-535, Environmental Research and Technology, Inc., Newbury Park, CA. 